\title{
Smart RAFT-Polymer Surfaces: \\ Exploring their Self-assembly and Dynamics on the Nanoscale
}

\author{
Dissertation \\ zur Erlangung des mathematisch-naturwissenschaftlichen Doktorgrades \\ "Doctor rerum naturalium" \\ der Georg-August-Universität Göttingen \\ im Promotionsprogramm Chemie \\ der Georg-August-University School of Science \\ (GAUSS) \\ vorgelegt von \\ Katharina Hendrich Geb. Dabow
}

Göttingen, 2020 


\section{Betreuungsausschuss}

Prof. Dr. Philipp Vana, MBA

Prof. Dr. Marcus Müller

\section{Mitglieder der Prüfungskommission}

\section{Referent:}

Prof. Dr. Philipp Vana, MBA Institut für Physikalische Chemie

Korreferent:

Georg-August-Universität Göttingen

Prof. Dr. Marcus Müller I. Physikalisches Institut

Georg-August-Universität Göttingen

Weitere Mitglieder der Prüfungskommission:

Prof. Dr. Michael Buback Institut für Physikalische Chemie Georg-August-Universität Göttingen

Prof. Dr. Burkhard Geil Institut für Physikalische Chemie Georg-August-Universität Göttingen

Prof. Dr. Jörg Enderlein III. Physikalisches Institut Georg-August-Universität Göttingen

Prof. Dr. Andreas Janshoff Institut für Physikalische Chemie Georg-August-Universität Göttingen

Tag der mündlichen Prüfung: 11.02.2020 


\section{Abstract}

Synthetic polymers on surfaces are excellent models to mimic biological systems which are highly sophisticated in their responsive behaviour to environmental changes. To expand the application possibilities of smart polymer surfaces as modern devices like sensors, microfluidic channels or even next-generation computer chips, the development of new surface analyzing techniques is required. In the context of this thesis, tethered polymers were synthesized and comprehensively analyzed using metal induced energy transfer (MIET) to establish this technique in polymeric surface science. With the gained insight of surface-tethered polymers, the generation of nanostructures mediated by constrained dewetting of homopolymers was investigated.

MIET is a novel method that allows for the measurement of accurate distances at the scale of macromolecular systems using fluorescence lifetime imaging. This technique offers the possibility to investigate single molecules attached to a silicon dioxide spacer on a thin gold layer. To exploit this potential, the preparation of structurally well-defined polymer chains is indispensable. Using reversible addition-fragmentation chain transfer (RAFT) polymerization, the goal of synthesizing immobilized polymer chains of desired grafting density, molar mass and end-group functionalization could be accomplished. Specifically, by the thorough investigation of different reaction conditions, optimum polymerization parameters were established to prepare MIET substrates with tethered poly(2-(dimethylamino)ethyl prop-2-enoate) ( $\mathrm{p}(\mathrm{DMAEMA}))$, a $\mathrm{pH}$-responsive polymer. This achievement provided the basis for exploring the scope of MIET in collaboration with the Enderlein group (Third Institute of Physics, University Göttingen).

For the first time, MIET measurements could successfully be conducted for polymer layers with grafting densities that range from individual tethered chains to high density polymer brushes. It was observed, that the layer thickness increases with increasing grafting density, which is in agreement with theoretical descriptions known from the literature. In order to 
explore the range of applicability of the MIET technique, $p$ (DMAEMA) was exposed to aqueous solutions at different $\mathrm{pH}$ values and measurements were performed to investigate the responsive polymer properties. Here, monitoring of repulsive segment-segment interactions for small grafting densities and additional repulsive interactions between adjacent polymer chains was achieved. The results of the MIET measurements were also confirmed by spectroscopic ellipsometry.

Within the second project of this thesis, it could be demonstrated that only a few tethered homopolymer chains are required to generate tailored nanostructured surfaces. The systems of choice were linear and four-arm star polystyrene samples prepared by RAFT polymerization on gold substrates. Without activation or chemical modification of the gold substrate, polymeric molecules of both architectures could be immobilized only via their sulfur-containing RAFT group. Subsequently, by means of an optimized dewetting technique, a variety of spherical and worm-like micelles as well as network structures were obtained. The nanostructures were specifically tuned by the adjustment of different grafting densities and the solvent quality during the dewetting procedure.

As the visual evaluation of the formed morphologies can be misleading, a strategy to quantitatively analyze the differently shaped domains was developed. An unambiguous characterization was achieved using Minkowski quantities and completed by the specification of the obtained domains through calculation of circularities.

Furthermore, the assembly of gold nanoparticles on planar gold substrates featuring polystyrene layers. Gold nanoparticles stabilized in aqueous solution could be precisely arranged within a preformed polymeric nanostructure. The latter prevented the uncontrolled aggregation of the gold nanoparticles, and instead mediated the formation of a dense nanoparticle monolayer. In contrast, the immersion of uniform polystyrene layers in dispersions of gold nanoparticles in toluene yielded multicomponent aggregates of tunable size, which could be controlled by the number of tethered polymer molecules. 


\section{Contents}

Abstract i

List of Figures $\quad$ v

1 Introduction and motivation 1

2 Theoretical background 3

2.1 Polymers on surfaces . . . . . . . . . . . . . . . . . 3

2.1.1 From mushroom to brush regime . . . . . . . . . . . 4

2.1.2 Grafted polymer exposed to solvent . . . . . . . . . . . 6

2.2 Analytical methods $\ldots \ldots \ldots \ldots$. . . . . . . . . . 8

2.2.1 Image analysis using Minkowski quantities . . . . . 8

2.2 .2 Spectroscopic ellipsometry $\ldots \ldots \ldots$. . . . . . . . . . . . . . . . 10

$2.2 .3 \quad$ Fluorescence microscopy $\ldots \ldots$. . . . . . . . . . 12

3 Polymers on surfaces: Let's talk about lifetime 19

3.1 Preface . . . . . . . . . . . . . . . . . . . 19

3.2 Tuning the process of SI-RAFT polymerization . . . . . . . . . 20

3.2.1 Basic strategy and mechanism . . . . . . . . . . . 20

3.2.2 Preparation of ultraflat silicon substrates . . . . . . . . 22

3.2.3 Tailoring the grafting density . . . . . . . . . . . . . 24

3.2.4 RAFT polymerization of DMAEMA . . . . . . . . . . . 32

3.2.5 SI-RAFT polymerization of DMAEMA . . . . . . . . 35

3.3 Fluorescence microscopy $\ldots \ldots \ldots$. . . . . . . . . 37

3.3 .1 Fundamentals . . . . . . . . . . . . . . . . . . . 39

3.3 .2 MIET measurements . . . . . . . . . . . . . . . . . . . . . . .

3.4 Closing remarks to Chapter 3 . . . . . . . . . . . . 47

4 Bringing structure into the world of polymers 49

4.1 Preface . . . . . . . . . . . . . . . . . . . . . . 49 
4.2 Fundamentals . . . . . . . . . . . . . . . . . . . 52

4.2.1 Preparation of ultraflat metal surfaces . . . . . . . . 52

4.2.2 Characterization of thin polystyrene layers . . . . . . . 55

4.2.3 Preliminary studies on constrained dewetting . . . . . 59

4.2.4 Wettability of nanostructured surfaces . . . . . . . 66

4.2 .5 Conclusion . . . . . . . . . . . . . . 68

4.3 Tunable nanostructures mediated by RAFT polymers . . . . . 68

4.3.1 Linear polymer systems . . . . . . . . . . . . 71

4.3 .2 Star polymer systems . . . . . . . . . . . . . . . . . . 81

4.3 .3 Conclusion $\ldots \ldots \ldots$. . . . . . . . . . . . . . 90

4.4 Selective self-assembly of AuNPs . . . . . . . . . . . . . . . . . 91

$4.4 .1 \quad$ Preface . . . . . . . . . . . . . . . . . . . 91

4.4.2 AuNP assemblies via polymeric templates . . . . . . . 95

4.4.3 Tunable composite nanostructures on gold surfaces . . 97

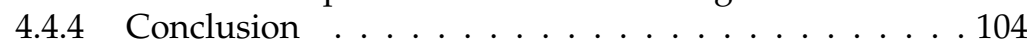

4.5 Closing remarks to Chapter 4 . . . . . . . . . . . 105

$\begin{array}{lll}5 & \text { Closing remarks and outlook } & 107\end{array}$

6 Experimental section 113

6.1 Instrumental setups . . . . . . . . . . . . . . . . . . . . 113

6.2 Materials . . . . . . . . . . . . . . . . . 116

6.3 Substrate preparation . . . . . . . . . . . . . . . . . . . 117

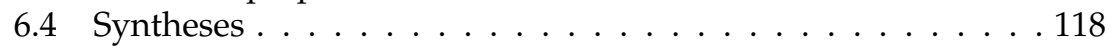

6.4.1 Activation of RAFT agent 4-cyano-4-[(dodecylsulfanylthiocarbonyl)sulfanyl]pentanoic acid $\ldots$. . . . . . 118

6.4.2 SI-RAFT Polymerization from silicon substrates . . . . 119

6.4.3 Substrate preparation for dynamic MIET measurements 120

6.4.4 Syntheses and substrate preparation for the design of nanostructures . . . . . . . . . . . . . 122

\begin{tabular}{ll}
\hline Appendix & 125
\end{tabular}

A. Additional figures and tables . . . . . . . . . . . . . . . . 125

B. Abbreviations . . . . . . . . . . . . . . . . . . . 138

\begin{tabular}{ll}
\hline Bibliography & 141
\end{tabular}

\begin{tabular}{ll}
\hline Acknowledgements & 149
\end{tabular}

$\begin{array}{ll}\text { About the author } & 151\end{array}$ 


\section{List of Figures}

$2.1 \quad$ Preparation strategies for polymeric surfaces . . . . . . . . . . . 6

2.2 Grafted polymer exposed to solvent . . . . . . . . . . . . 8

2.3 Minkowski quantities.$\ldots \ldots \ldots$. . . . . . . . . . . 10

2.4 Ellipsometry setup . . . . . . . . . . . . . . . . . . . 11

2.5 Principle of ellipsometry $\ldots \ldots \ldots \ldots 11$

2.6 Principle of fluorescence . . . . . . . . . . . . . . . . 13

2.7 Principle of TCSPC $\ldots \ldots \ldots \ldots \ldots \ldots$

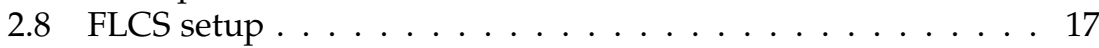

2.9 Principle of MIET . . . . . . . . . . . . . . . . . . . . 18

3.1 Initial RAFT equilibrium . . . . . . . . . . . . . . . . . . . 21

3.2 SI-RAFT polymerization . . . . . . . . . . . . . . . 22

3.3 WCA of OH-terminated surface . . . . . . . . . . . . . . 23

3.4 Variation of silicon dioxide layer thickness . . . . . . . . . . . 24

3.5 Grafting-to approach of PMA $\ldots \ldots \ldots$. . . . . . . . . . . . . . . 26

3.6 From mushroom to polymer brush . . . . . . . . . . . . . 27

3.7 Tailoring the grafting density $\ldots \ldots \ldots$. . . . . . . . . . . . . . . . . . . . . . . . . 30

3.8 RAFT agent and RAFT mask . . . . . . . . . . . . . . . . . 30

3.9 Inluence of RAFT mask $\ldots \ldots$. . . . . . . . . . . . . 31

3.10 RAFT agents with different R-groups . . . . . . . . . . . . . . 32

3.11 Influence of R-group composition II . . . . . . . . . . . . . . . . . . . . . . . . . . .

3.12 Influence of R-group composition $\ldots \ldots$. . . . . . . . . . . 35

3.13 Si-RAFT polymerization of DMAEMA . . . . . . . . . . . . 36

3.14 WCA measurements . . . . . . . . . . . . . . . . 37

3.15 Polymer conformations . . . . . . . . . . . . . . . 38

3.16 Custom-built reaction flask . . . . . . . . . . . . . . . . 40

3.17 Fluorescence intensity scan image . . . . . . . . . . . . . . . . . . 4 40

3.18 MIET curve . . . . . . . . . . . . . . . . . . . . 41

3.19 FLIM with corresponding TCSPC curve . . . . . . . . . . . . 42

3.20 MIET with corresponding TCSPC curve . . . . . . . . . . . . . 44 
3.21 Swollen polymer chains . . . . . . . . . . . . . . 47

4.1 Simulated nanostructures . . . . . . . . . . . . 50

4.2 Binding modes of RAFT agents on gold surfaces . . . . . . . . 51

4.3 Evaporated gold layer on glass substrates $\ldots$. . . . . . . . . . 53

$4.4 \quad$ Thin gold layer before and after plasma treatment . . . . . . . 55

4.5 Reduced grafting density of polystyrene . . . . . . . . . . 57

4.6 Binding modes of linear and star shaped polystyrene . . . . . 58

$4.7 \quad$ Principle of constrained dewetting . . . . . . . . . . . . . 6 60

$4.8 \quad$ Dewetting, fast and slow $\ldots \ldots \ldots$. . . . . . . . . . . . . . . . . . 62

4.9 Dewetting, high and low temperature . . . . . . . . . . . . 64

4.10 Dewetting, thermal stability . . . . . . . . . . . . . . 65

4.11 Wettability of nanostructured surfaces . . . . . . . . . . . . 6 67

4.12 Principle of nanostructure specification . . . . . . . . . . . 70

4.13 Dewetting, linear PS with $M_{\mathrm{n}}=21 \mathrm{~kg} \mathrm{~mol}^{-1} \ldots$. . . . . . . . 73

4.14 Dewetting, linear PS with $M_{\mathrm{n}}=40 \mathrm{~kg} \mathrm{~mol}^{-1} \ldots . . . . . .775$

4.15 Maximum height of nanostructures $\ldots . . . . . . . .776$

4.16 Dewetting, linear PS with $M_{\mathrm{n}}=64 \mathrm{~kg} \mathrm{~mol}^{-1} \ldots$. . . . . . . 77

4.17 Segmentation by circularity, linear PS with $M_{\mathrm{n}}=64 \mathrm{~kg} \mathrm{~mol}^{-1} 78$

4.18 Dewetting with different solvent qualities . . . . . . . . . . . . 79

4.19 Network fragments . . . . . . . . . . . . . . . . . . 80

4.20 Tailoring the morphology . . . . . . . . . . . . . . . . . . . 81

4.21 Dewetting, star PS with $M_{\mathrm{n}}=22 \mathrm{~kg} \mathrm{~mol}^{-1} \ldots . . . . . .883$

4.22 Perimeter expansion $\ldots \ldots \ldots \ldots$. . . . . . . . . . . . . 84

4.23 Dewetting, star PS with $\bar{M}_{\mathrm{n}}=60 \mathrm{~kg} \mathrm{~mol}^{-1} \ldots$. . . . . . . . . 85

4.24 Nanostructures with different polymeric architectures . . . . 86

4.25 Nanostructures of different reduced grafting densities . . . . 88

4.26 Dewetting, star PS with $M_{\mathrm{n}}=84 \mathrm{~kg} \mathrm{~mol}^{-1} \ldots \ldots$. . . . . . 89

4.27 Distribution AuNPs . . . . . . . . . . . . . . . . 93

4.28 Tip artifacts during AFM . . . . . . . . . . . . . . . . . . . . . . . . . . . . . . . 94

4.29 Arrangement of AuNPs . . . . . . . . . . . . . . . . 96

4.30 AuNPs embedded in polymeric nanostructure . . . . . . . . . 97

4.31 Reversibility of composite nanostructure with $\Sigma=2.9$. . . . 98

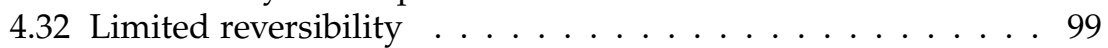

4.33 AuNPs assembled in star PS with $\bar{M}_{\mathrm{n}}=22 \mathrm{~kg} \mathrm{~mol}^{-1} \ldots . . .100$

4.34 Schematic depiction of multicomponent domains . . . . . . . 102

4.35 Multicomponent assemblies . . . . . . . . . . . . . . . . 103

$5.1 \quad$ MIET with corresponding TCSPC curve . . . . . . . . . . . 109 
6.1 Investigated monomers . . . . . . . . . . . . . . . . . . 117

6.2 Activation of RAFT agent . . . . . . . . . . . . . . . . . . . . 119

A1 Dewetting, linear PS with $\bar{M}_{\mathrm{n}}=64 \mathrm{~kg} \mathrm{~mol}^{-1} \ldots \ldots \ldots \ldots . .127$

A2 $\quad$ Dewetting, star PS with $M_{\mathrm{n}}=22 \mathrm{~kg} \mathrm{~mol}^{-1} \ldots \ldots$. . . . . . . 129

A3 Dewetting, star PS with $\bar{M}_{\mathrm{n}}=60 \mathrm{~kg} \mathrm{~mol}^{-1} \ldots \ldots \ldots \ldots . .130$

A4 Dewetting, star PS with $M_{\mathrm{n}}=84 \mathrm{~kg} \mathrm{~mol}^{-1} \ldots \ldots \ldots$. . . . 131

A5 Additional multicomponent nanostructures . . . . . . . . . . . 132

A6 $\quad$ Fragmentation of multicomponent nanostructures . . . . . . . 133

A7 $\quad$ Nanomechanical mapping . . . . . . . . . . . . . . . . . . . . 134

A8 $\quad$ TEM, TOAB-capped AuNPs . . . . . . . . . . . . . . . . . . . . 135

A9 TEM, citrate-stabilized AuNPs . . . . . . . . . . . . . 136 



\section{Chapter 1}

\section{Introduction and motivation}

Isn't it fascinating how the microstructure of materials can dictate their bulk properties?

The change in hardness of carbon from graphite to diamond, the hardest natural material known, may be the most spectacular example. [1] Another one is gold which is often used as jewelry due to its lustrous appearance. Turning solid gold into colloidal solutions, however, the nanoparticles cause a deep red to bluish color of the solution. ${ }^{[2]}$ Besides this visual effect, gold drastically changes its reactivity in its nanosized form: Being chemically inert in its bulk form, nanosized clusters on a $\mathrm{TiO}_{2}$ support exhibit high catalytic activity in the partial oxidation of hydrocarbons. [3] This illustrates that especially the reduction of one or more dimensions to the nanoscale can result in intriguing material properties. Nanosized structures on surfaces can provide enhanced wear and corrosion resistance and when structural patterns are "written" on surfaces, they become highly promising for future generations of electronic devices. ${ }^{[1]}$ However, these patterns are prepared via lithography and hence rather expensive in their preparation.

In this regard, the functionalization of surfaces with polymers is advantageous. In principle, a multitude of options is available to decorate surfaces with polymers in a desired fashion. [4] The first degree of freedom is the grafting density that can be varied from dense and stretched polymer brushes to low density polymer chains that resemble the behavior of random coils. The other degree of freedom is based on the chemical nature of the polymers and their architecture. Each example naturally carries certain advantages and limitations that determine their application: Be it a rather simple goal as tuning the wetting properties, or stimuli-responsive 
polymer brushes that can change the wettability and binding properties to, for example, act as valves in microfluidics. [4]5]

RAFT polymerization is considered most versatile in the frame of surface functionalization because the RAFT process tolerates several functional groups and reaction conditions. [6] The flexibility in the design of polymer functionalized surfaces allows for applications in antifouling medical devices, antibacterial coatings and biomedical implants. 7$]$ Other interesting applications for polymer brushes are found in medicine and catalysis when they are used as carrier systems for nanoparticles. ${ }^{[8]}$ Therefore, it appears worthwhile to dedicate further research effort to this interesting class of composite materials.

This doctoral research study was initiated as part of the SFB 937. It was the goal of project A5 of the SFB to measure the vertical and parallel motion of fluorescent molecules that are attached to polymer brushes via metal-induced energy transfer and fluorescence correlation spectroscopy (MIET-FCS). The movement of the fluorescent molecules may then be interpreted in terms of shear-flow induced hydrodynamics within these brushes. Coupled with theoretical modelling the results should deliver valuable insight into the transport mechanics of biological systems. [9] To address the demands of this project adequately, it was indispensable to prepare polymers with tailored chain length and precisely tune their grafting density on planar surfaces. The scope of MIET-FCS measurements of polymer functionalized surfaces was explored.

Frequently, the functionalization of surfaces is achieved via immobilization of anchoring groups prior to polymer functionalization. The process is consequently more labor-intensive and leaves room for optimization. [6] Utilizing the expertise obtained in the SFB project, the other part of this work was therefore dedicated to the intriguing method of polymer dewetting as a means to produce nanostructured surfaces. Using polymers, prepared via RAFT polymerization, in combination with gold surfaces, the structuring process can also be conducted without a prior surface functionalization. The formed polymeric nanostructures were also investigated regarding their potential as templates for gold nanoparticle assemblies. 


\section{Chapter 2}

\section{Theoretical background}

\subsection{Polymers on surfaces}

Polymer chains grafted on a solid surface firstly became a subject of interest when van der Waarden and Mackor published their studies about the stabilization of inorganic nanoparticle dispersions. ${ }^{[10,11]}$ Macromolecules were tethered onto carbon black particles and prevented the flocculation of colloidal dispersions. Reports of theoretical approaches to explain the effect followed by Alexander and de Gennes. [12-14] Methods which were developed to characterize the surface helped to understand the behavior of polymers on surfaces. [15] Compared to free polymer chains in solution, tethered polymer chains show different properties and interesting effects, for example:

- Dense localization of terminal groups,

- diffusion control,

- response to external stimuli,

- wetting control,

- lubrication.

Nowadays, numerous detailed studies on grafted polymers led to their application in many fields, such as medicine, biophysics and surfactant science. [16] 
In the following sections an introduction to theoretical principles of grafted polymer chains is given. Additionally, structural definitions, preparation of polymer layers and the behavior under different solvent conditions are described.

\subsubsection{From mushroom to brush regime}

Grafted polymer chains on solid surfaces are categorized into three different regimes. The classification depends on the grafting density and consequently the distance of the grafting points of the polymer chains to each other.15] Single grafted chains with large distances lead to noninteracting polymer chains, which is often called the mushroom regime. With the decrease of the distance the polymer chains start to overlap and the interaction increases. This is the so called crossover regime. Polymer brushes are formed for very short distances between the grafting points. The grafting density is so high, that the polymer chains are compelled to stretch away from the surface to avoid repulsive steric interaction and overlapping. [17]

A quantitative parameter to describe the polymeric system regarding the dimensions of polymer molecules is the reduced grafting density $\Sigma$. The reduced grafting density $\Sigma$ is proportional to the root mean square radius of gyration $\left\langle s^{2}\right\rangle^{1 / 2}$ which depends on the experimental conditions like temperature and solvent. It yields the number of macromolecules that occupy a defined area on the surface compared to the area that a free noninteracting polymer chain occupies at the same experimental conditions. The reduced grafting density is given by: ${ }^{[18]}$

$$
\Sigma=\sigma \cdot \pi \cdot\left(\left\langle s^{2}\right\rangle^{1 / 2}\right)^{2},
$$

where $\sigma$ is the grafting density in chains per $\mathrm{nm}^{-2}$. Table 2.1 gives an overview over the theoretical classification of grafted polymer chains by the reduced grafting density $\Sigma$.

The transitions between these three regimes are not sharp, but depend on multiple factors, such as the polydispersity of the polymer or the solvent environment. Though the brush regimes are theoretically classified as shown in Table 2.1, a value of $\Sigma>5$ for high density polymer brushes is usually found in literature. ${ }^{[15]}$

The grafting of polymer chains onto a solid surface can be achieved by physisorption or chemisorption. ${ }^{[15]}$ Although the anchoring by physisorp- 
2.1 Polymers on surfaces

Table 2.1: Classification into three regimes of grafted polymer chains using the reduced grafting density $\Sigma$. A small $\Sigma$ leads to a polymer surface with non- or weaklyinteracting polymer chains. A large $\Sigma$ leads into a stretched conformation, that is called polymer brush.

\begin{tabular}{cc}
\hline & regime \\
\hline \hline$\Sigma<1$ & mushroom \\
$\Sigma \approx 1$ & crossover \\
$\Sigma>1$ & polymer brush \\
\hline
\end{tabular}

tion of the polymer chain is a very simple method, the covalent binding is more preferred due to the stability under different solvent and temperature conditions. [19]

There are three methods to prepare chemisorbed polymer chains by radical polymerization: Grafting-to, grafting-through and grafting-from. [20] Applying the grafting-to approach preformed polymers are anchored to the substrate via their chain-end functionality. ${ }^{[21]}$ In some cases a complementary functionalization of the surface is required, depending on the nature of the used surface. 20 ] During the grafting-through approach the surface is prefunctionalized with unsaturated monomer units, which can terminate with macromolecular radicals. Here, the propagation predominantly takes place in the polymerization solution. ${ }^{[22]}$ The grafting-from method is a bottom-up approach, because polymer chains are growing via surface-initiated polymerization. Within this approach the surface is immobilized with functional groups which can serve as an initiator for the polymerization.

The mostly used techniques are the grafting-to and grafting-from approach. Polymeric surfaces prepared via grafting-to are often limited in their grafting densities due to the steric hindrance of already anchored polymer chains. The limitation mainly depends on the molar mass of the preformed polymer, so that higher molar masses lead to even lower grafting densities. ${ }^{[23]}$ To synthesize a high density polymer brush grafting-from is the preferred technique. Figure 2.1 illustrates the three possible methods to prepare polymeric surfaces.

It has to be noted that in Figure 2.1 the grafting-from approach is presented for the reversible addition-fragmentation chain transfer (RAFT) polymerization, but works similar for other polymerization techniques. The controlling agent, called RAFT agent, is immobilized to the surface via 


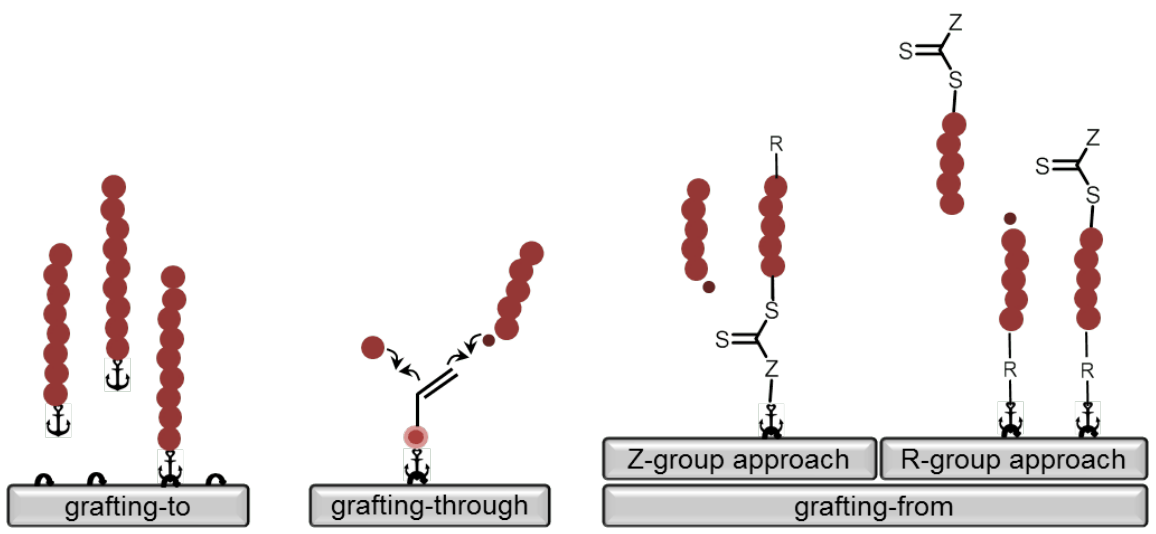

Figure 2.1: Synthesis strategies to covalently bind polymer chains onto a substrate. Three procedures are possible: Grafting-to, grafting-through and grafting from.

its Z-group or R-group, which terms the approach Z-group and R-group approach, respectively. Due to its mechanism the Z-group approach can also be regarded as a grafting-to approach. ${ }^{20]}$ The polymer chains are growing in solution and can be anchored to the surface by the exchange with the R-group of the RAFT agent. For further details about the RAFT polymerization technique and its controlled character, the reader is referred to literature. ${ }^{[24-27]}$ In the R-group approach propagating chains are anchored to the surface. Since the propagating radicals remain at the outer sphere of the polymer film, the highest grafting densities can be achieved using this method. $[28]$

To enhance the controlled character of the RAFT polymerization a so called sacrificial RAFT agent is typically added to the polymerization mixture. [20]

\subsubsection{Grafted polymer exposed to solvent}

Unattached polymer chains in solution are well described by the FloryHuggins theory. ${ }^{29,30]}$ The Gibbs free energy $\Delta G_{\mathrm{m}}$ is predicted for mixing by considering polymer chains as consisting of segments. Each chain segment has the same size as a solvent molecule. The Gibbs energy of mixing at constant temperature and pressure is given by the Gibbs-Helmholtz equation: [31] 


$$
\Delta G_{\mathrm{m}}=\Delta H_{\mathrm{m}}-\mathrm{T} \Delta S_{\mathrm{m}}
$$

where $\Delta H_{\mathrm{m}}$ is the change of the enthalpy of mixing, $T$ the temperature and $\Delta S_{\mathrm{m}}$ the change of the entropy of mixing.

For polymer mixtures the enthalpic contribution results from the interactions between the molecules. The entropic contribution is described by the conformation of the polymer chains in the solution. ${ }^{[32]}$ The absence of interactions would lead to $\Delta H_{\mathrm{m}}=0$ and an ideal entropy of mixing. The entropy of mixing under ideal conditions always has a positive value, so that the free energy becomes negative. Every mixture independent of the mixing ratio is miscible. Based on this considerations, miscibility gaps have to result from interactions between the molecules. To address this, the free Gibbs energy additionally has to consider segment-segment, solvent-solvent and segment-solvent interactions. Flory and Huggins set up the following equation: [29]

$$
\Delta G_{\mathrm{m}}=\mathrm{R} T\left[n_{1} \ln \phi_{1}+n_{2} \ln \phi_{2}+n_{1} \phi_{2} \chi_{12}\right],
$$

where $n_{\mathrm{i}}$ is the number of moles, $\phi_{\mathrm{i}}$ the volume fraction and $\chi$ the FloryHuggins parameter. This parameter is a temperature dependent quantity to describe the segment-solvent interaction. [29]

Obviously, the value of $\chi$ has to change for polymer solutions with different solvent environments. ${ }^{[32]}$ In a good solvent the interactions between the polymeric segments and solvent molecules are more attractive than the interactions between the polymeric segments themselves. To maximize the contact to the solvent and simultaneously minimize the segment-segment intramolecular interactions the polymer coil expands. ${ }^{[32]}$ When polymer chains are exposed to a poor solvent the segment-solvent interactions become unfavorable. The contraction of polymer chains occurs to minimize the contact between the segments and solvent molecules. [29] Thus, two contrary tendencies occur: The expansion of a polymer coil to avoid longrange intramolecular steric interactions and the contraction to minimize the number of contacts between segments and solvent molecules. A special case is the mixture under so called $\theta$-conditions. Here, the dissolved polymer chains behave like ideal chains. The favored segment-solvent interactions completely counteract the repulsive segment-segment interactions. Under these conditions the solvent is termed $\theta$-solvent. ${ }^{29]}$

Surface-attached polymers exposed to a solvent show a different behavior due to their constraint regarding the attachment. The most favorable 
conformation of a polymer chain is the ideal chain, which can freely stretch in a random walk. ${ }^{32]}$ However, attached to a surface and exposed to a good solvent they can maximize their entropic contribution and aspire a random walk or stretch to maximize the polymer-solvent interactions. ${ }^{[16]}$ Due to the proximity of the polymer chains to each other the polymeric system is balanced between these two contrary tendencies. The exposition to a poor solvent leads to the contraction of the polymer coils. ${ }^{[33}$ Under poor solvent conditions, the consideration of the grafting density is required. A high density polymer brush forms a uniform polymer layer to exclude solvent molecules. Sparsely attached polymer chains collapse on the surface and a lateral structure is formed, which depends on the number of tethered chains. ${ }^{[34]}$ Very low grafting densities lead to isolated micelles, which can grow to a network with increasing grafting density. [35,36]

Figure 2.2 illustrates the resulting conformations under good and poor solvent conditions. Additionally, the height of the respective structures is marked by light grey areas around the polymer chains. The height represents the balance between steric segment-segment interactions and segment-solvent interactions.

height

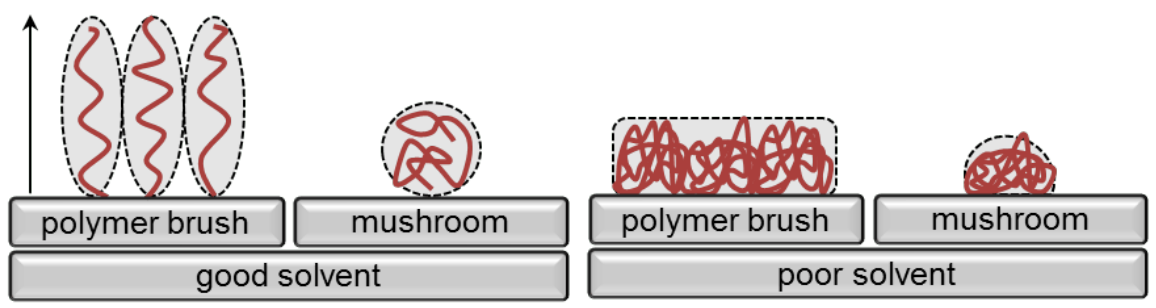

Figure 2.2: Illustration to highlight resulting conformations of polymer brushes and tethered polymer chains in the weakly-interacting regime under good and poor solvent conditions.

\subsection{Analytical methods}

\subsubsection{Image analysis using Minkowski quantities}

Within this PhD study homopolymer films on planar substrates were investigated. Under certain solvent conditions the polymer films formed 
characteristic nanostructures. The surface characterization was conducted by atomic force microscopy (AFM), where topographic images were obtained. The quantitative analysis of the morphological differences of the AFM images was achieved by the calculation of Minkowski quantities. The Minkowski quantities are commonly used in digital picture analysis and integral geometry. $37-39]$

AFM images are constructed of numerous pixels, with each containing one specific height information. By the conversion of each pixel into black or white, depending on whether they are larger or lower than a defined threshold variable, level contours are formed. ${ }^{40]}$ The irregularities within the black-and-white images arise from the corners of the pixels, which can be described by three Minkowski quantities. [37]

Figure 2.3 represents a part of a black-and-white image to illustrate a possible arrangement of grey and white pixels shown as squares. The grey pixels represent pixels with a larger value than the defined threshold $t$. The arrangement of the pixels constructs corners, which can be described by the Minkowski quantities $f(t), s(t)$ and $\chi_{\mathrm{E}}$. The quantity $f(t)$ is the area fraction of pixels that have a larger value than $t$, here the grey pixels. The length of boundary line $s_{\mathrm{t}}$ illustrates the number of boundaries $B$ of neighbored grey and white pixels divided by the total number of pixels $N$. The third quantity is described by the Euler characteristic $\chi_{\mathrm{E}}(t)$ which represents the difference of grey $N_{t}^{g}$ and white $N_{t}^{w}$ components divided by the total number $N$. Alternatively, the Euler characteristic can be calculated by adding all curvature values given in Figure 2.3 as small numbers. When the two nearest neighbors joining at the corner are white, the value is -1 , if they are grey the value is +1 . In both cases the next-nearest has to be white. For every other case the curvature value is 0 . 37

Table 2.2: Minkowski quantities to describe morphological differences in digital blackand-white images.

\begin{tabular}{cl}
\hline quantity & description \\
\hline \hline$f(t)$ & area fraction with $f(t)=N_{\mathrm{t}} / N$ \\
$s(t)$ & length of boundary line with $s_{\mathrm{t}}=B_{\mathrm{t}} / N$ \\
$\chi_{\mathrm{E}}(t)$ & Euler characteristic with $\chi_{\mathrm{E}}(t)=\left(N_{\mathrm{t}}^{g}-N_{\mathrm{t}}^{w}\right) / N$ \\
\hline
\end{tabular}




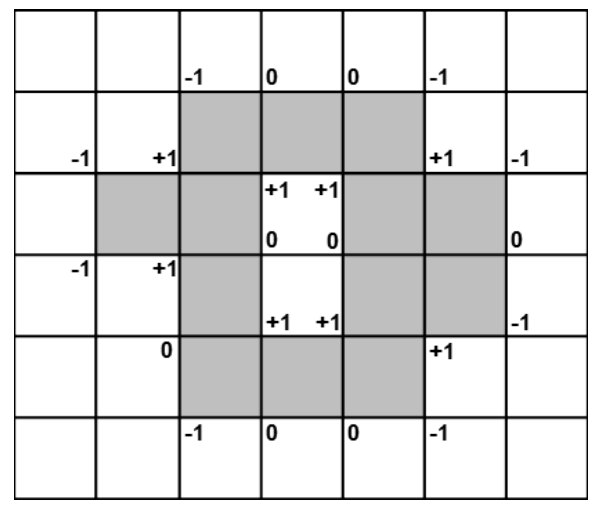

Figure 2.3: Illustration of a black-and-white image with local curvature values at the corners. The application of the Minkowski measures yields $f(t)=\frac{13}{42}$ for the area fraction and $s(t)=\frac{4}{7}$. The Euler characteristic $\chi_{\mathrm{E}}(t)$ equals 0 . Please note that, by definition, the surrounding white region is not counted as a white component.

\subsubsection{Spectroscopic ellipsometry}

Characteristics of the method and the experimental setup were adapted from the Handbook for nanofilm EP4 kindly provided by Accurion GmbH. ${ }^{[1]}$

Ellipsometry is an optical technique that allows for determination of dielectric properties such as optical constants and especially the thickness and composition of reflecting surfaces or thin films. The technique is highly sensitive to the surface state and, consequently, basic research and industrial applications established numerous uses for ellipsometry. The fields of interest include the chemical formation of polymers films, semiconductor growth and microelectronics. ${ }^{[4]}$

The operating principle is based on the measurement and tracking of polarized light. When polarized light is reflected from a surface, the state of polarization changes. The analysis of the change yields information about thin layers which can exhibit a thickness of only a fraction of the wavelength of the probing light. Figure 2.4 shows the basic components of an ellipsometer. The most crucial parts are the polarization state generator (PSG) and polarization state analyzer (PSA) that control and analyze the polarization states of the incident and reflected light, respectively. 


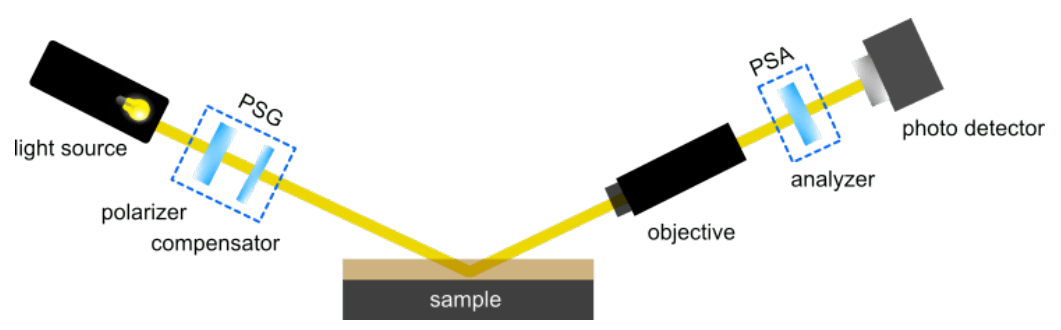

Figure 2.4: Basic setup of a spectroscopic ellipsometer.

A beam of polarized light hits a sample under an oblique angle of incidence. Due to the reflectivity of the sample, a phase change of the probing beam is induced. The phase change is different for the parallel and perpendicular components of the light beam relative to the plane of incidence (see Figure 2.5). The total reflection coefficients of the incident beam are denoted by $R_{\mathrm{p}}$ for the parallel component and $R_{\mathrm{s}}$ for the perpendicular component. $\delta_{\mathrm{i}}$ and $\delta_{\mathrm{r}}$ indicate the phase differences of the incident beam and the reflected beam. The relative phase changes of the $\mathrm{p}$ - and s-components and the reflectivity ratio are determined and converted to ellipsometry angles $\Psi$ and $\Delta$ :

$$
\begin{aligned}
& \tan \Psi=\frac{R_{\mathrm{p}}}{R_{\mathrm{s}}} \\
& \Delta=\delta_{\mathrm{i}}-\delta_{\mathrm{r}}
\end{aligned}
$$

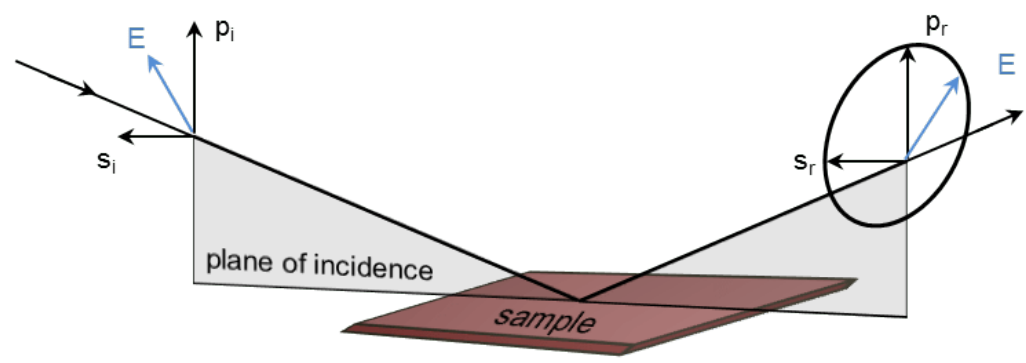

Figure 2.5: Illustration to demonstrate the principle of ellipsometry. The incident light beam is exemplarily shown as linearly polarized. 
Due to the operating mode of the ellipsometer used in this work, the method is called nulling ellipsometry: For a specific sample, the incident light is tuned to such an elliptical state of polarization that it will generate a linearly polarized reflection. The analyzer (PSA, see Figure 2.4) can then be set to a $90^{\circ}$ position with respect to the axis of the linear polarization which ultimately extinguishes the beam. Correspondingly, the process is termed "nulling".

\subsubsection{Fluorescence microscopy}

\section{Phenomenon of fluorescence}

During the phenomenon of fluorescence, light is absorbed and re-emitted directly after the excitation. ${ }^{43 \mid}$ Light always occurs in quantized energy portions, called photons. A Jablonski diagram can be used to describe a fluorescence process regarding the quantum mechanical principles, see Figure 2.6. The interaction with light temporarily changes the electronic state of the system. A fluorescent system can be illustrated as a system consisting of two electronic states, a singlet ground state $S_{0}$ and singlet first state $S_{1}$. Each electronic state consists of additional substates, the vibrational energy levels, that are illustrated as dotted lines in Figure 2.6. The excitation to a higher electronic energy level requires the absorption of a photon, which corresponds in the amount of energy to the difference between the two states. However, the excitation can also lead into the vibrational substates. The relaxation into the ground state can lead to an emission of a fluorescent photon, which corresponds to a radiative transition. A non-radiative transition can occur when the electron relaxes into another excited state without the emission of a photon or the absorbed energy is converted into heat. These two possible transitions lead to an energetic shift between the absorption and emission spectrum, which is called Stokes shift. The absorption and emission spectra of Атто655 are shown in picture (b) in Figure 2.6.

Besides the absorption and emission spectra further characteristics to describe a fluorophore are the fluorescence lifetime and quantum yield. The lifetime refers to the average time that a fluorophore stays in the excited state before returning to the ground state. As already mentioned two electronic transitions from the singlet first to the singlet ground state can occur. The radiative and non-radiative transition are given by the transition rates $k_{\mathrm{r}}$ 


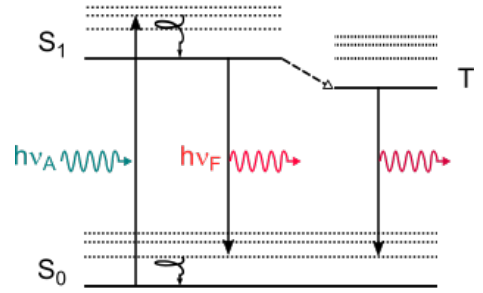

(a)

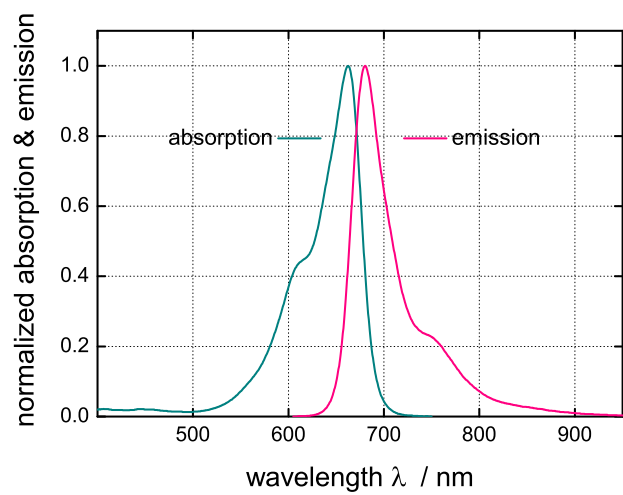

(b)

Figure 2.6: Illustration to highlight the fluorescence process. Picture (a) shows a simplified Jablonski diagram, which describes the excitation of a system through the absorption of quantized light. The energy can be released in form of a fluorescent photon. Picture (b) shows the Stokes shift resulting from radiative and non-radiative transitions.

and $k_{\mathrm{nr}}$, respectively. Thus, the fluorescence lifetime $\tau$ can be described as follows: ${ }^{43]}$

$$
\tau=\frac{1}{k_{\mathrm{r}}+k_{\mathrm{nr}}}
$$

The fluorescence quantum yield QY is described by the ratio of emitted photons and absorbed ones, following equation 2.7. 43.

$$
\mathrm{QY}=\frac{k_{\mathrm{r}}}{k_{\mathrm{r}}+k_{\mathrm{nr}}}=k_{\mathrm{r}} \tau \text {. }
$$

Here, the number of absorbed photons is a combination of the two transition rates $k_{\mathrm{r}}$ and $k_{\mathrm{nr}}$. The quantum yield states the probability of a radiative process. The quantum yield also defines, in addition to the extinction coefficient, how bright the fluorescent system can appear. [44]

In the Jablonski diagram in Figure 2.6 several interactions are excluded to highlight the fluorescence phenomenon. However, one additional state is illustrated, because it often appears during fluorescence spectroscopy measurements. It belongs to the so called forbidden transitions, because 
the system undergoes a spin conversion from the singlet first state $S_{1}$ to the triplet state $\mathrm{T}$. The transition from one excited main state to another is called inter-system crossing (ICS). The associated transition rate $k_{\text {ICS }}$ is several orders of magnitude lower than for the transition between the singlet states. If the system is located in the triplet state, the emitter is dark. This process can often be observed as a blinking of a fluorophore.

Experimentally the fluorescence lifetime can be determined by the excitation of the fluorescent system using a laser pulse and detecting the emitted photons in a time-resolved manner. The starting point is defined by the excitation laser pulse. From that moment on, the time is recorded until the arrival of the emission photon at the detector system. Time correlated single photon counting (TCSPC) represents a suitable experimental setup to determine the lifetime and is explained in the following section.

\section{Time correlated single photon counting}

In addition to the absorption and emission spectra, time-resolved measurements yield further information about fluorescent systems. Using time-resolved spectroscopy the studied sample is excited by short laser pulses and the fluorescence intensity decay dependent on time can be determined. Here, the direct detection of the time-dependent decay is challenging.

Fluorescence processes typically last several nanoseconds, which consequently have to be recorded with a temporal accuracy of picoseconds. [45],46] To record the whole decay curve and not only a single fluorescence lifetime a high quantity of emitted photons is required. The measurement of the intensity decay would be impossible by the detection of only one excitation cycle of one emitter. The development of time correlated single photon counting (TCSPC) overcomes these challenges. Using a periodic laser excitation data over multiple excitation and emission cycles can be collected, so that the profile of the intensity decay can be reconstructed.

Figure 2.7 illustrates the reconstruction of a decay profile by TCSPC. It is based on the precisely temporal detection of emitted single photons, which are referenced to the excitation laser pulse. The small green profile represents the excitation laser pulse, which generates the large fluorescence intensity decay, illustrated in red. The small red profiles represent the detected emitted photons, which are summed in a histogram. The photon counting histogram depicts a fluorescence decay after a high number of ex- 
citation periods. The principle of TCSPC shown in Figure 2.7 is simplified to highlight the reconstruction of the fluorescence intensity profile. Normally, the TCSPC setup is adjusted, so that less than one photon is recorded after one excitation pulse on an average. Typical counts rates are approximately 1 photon per 100 excitation pulses. ${ }^{[43]}$ Larger count rates lead to a bias of shorter fluorescence lifetimes.

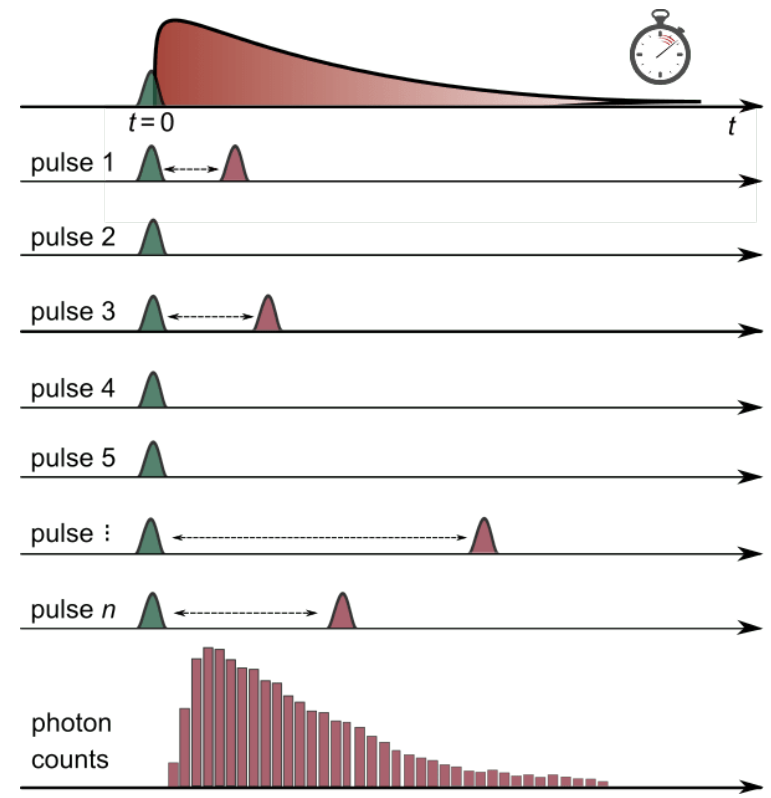

Figure 2.7: Illustration to highlight the principle of time correlated single photon counting. Excitation laser pulses (small green profiles) lead to single photons (small red profiles), which can be detected dependent on time. The histogram of all counted photons reconstructs the fluorescence intensity decay.

Sensitive detectors for single photon counting are the photomultiplier tube (PMT) and the single-photon avalanche photodiode (SPAD). ${ }^{[43]}$ PMTs typically show low counting efficiencies between $10 \%$ and $40 \%$, which is limited by the frequent generation of a photoelectron by the photoelectric effect of an incident photon. This efficiency is termed quantum efficiency. Using SPADs photon counting with high efficiencies of over $70 \%$ and low noise are realized. [47] However, a smaller active area can complicate the measurement. 


\section{Fluorescence correlation spectroscopy}

Fluorescence correlation spectroscopy (FCS) was firstly introduced in 1972 by Magde, Elson and Webb. ${ }^{[48]}$ Using this method they were able to explore thermodynamic concentration fluctuations within the binding of ethidium bromide to DNA. Only two years later further detailed publications were published, where the theoretical background and experimental applicability of FCS was outlined. ${ }^{[4,50]}$ Since then, biologists, biophysicists, chemists and physicists applied this experimental technique to investigate fluctuations of fluorescence in dynamic molecular systems. ${ }^{[51-53]}$ FCS exhibits several advantageous properties, such as: [53]

- Small amounts and low concentrations of sample are required,

- investigation of in situ measurements is possible,

- simultaneous observation of different systems depending on their fluorescent label.

During an FCS experiment, fluorescent systems are typically excited by continuous wave excitation. After the record of the fluorescence intensity fluctuations a correlation analysis is applied. A continuously fluctuating fluorescence signal can be identified as a steep decrease in a correlation curve. ${ }^{[54]}$ Intensity fluctuations within fluorescent systems can be observed for example during chemical reactions, photophysical transformations or conformational changes. The obtained correlation time is connected to the respective chemical rate or diffusion constants.

The combination of TCSPC and FCS leads to fluorescence lifetime correlation spectroscopy (FLCS). ${ }^{[55,56]}$ Here, a separate correlation analysis for each recorded fluorescence lifetime component is conducted, which can be detected from several fluorescent species within the sample. The setup for FLCS resembles the experimental setup for FCS and is shown in Figure 2.8. It consists of a confocal microscope equipped with a TCSPC device and an FCS device. A repetitive laser pulse is focused by an objective lens through a small confocal volume through the fluorescent sample. The emitted light is again collected by the objective and separated from the excitation laser beam using a dichroic mirror. The fluorescent beam is collimated through a micrometer-sized pinhole and focused onto two single-photon avalanche photodiodes using a beam splitter. 


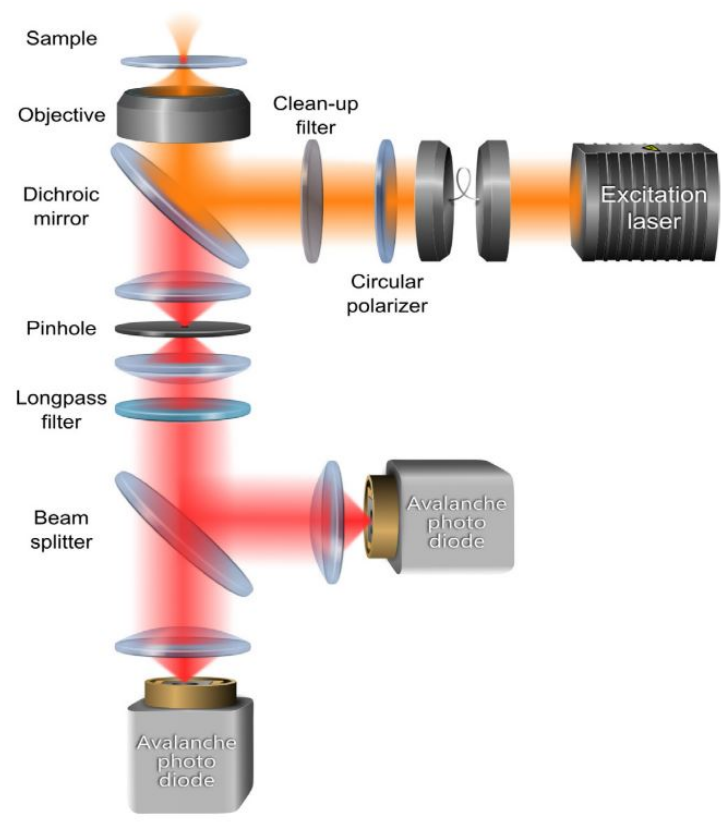

Figure 2.8: Schematic depiction of a setup for fluorescence lifetime correlation spectroscopy. ${ }^{[54]}$ Adapted with permission from A. Ghosh et al., Methods 2018, 140-141, and used without modification.

FLCS is an extended FCS method and can be applied when information about specific fluorescence lifetimes or fluctuations of fluorescence lifetime within the system is required.

\section{Metal-induced energy transfer}

The fundamental phenomenon behind metal-induced energy transfer (MIET) is the Förster resonance energy transfer (FRET). ${ }^{[57]}$ FRET is based on the non-radiative energy transfer from an excited fluorescent donor to an absorbing acceptor molecule. Theodor Förster illustrates in his publication from 1948 that the efficiency of energy transfer depends on the sixth power of the donor-acceptor distance. ${ }^{[58]}$ This distance dependence made FRET a powerful tool within fluorescence analytics to measure distances on the molecular scale up to approximately $10 \mathrm{~nm}$. 
The replacement of the acceptor molecule with a metal surface showed a similar effect to the phenomenon of FRET. ${ }^{[59]}$ However, the plane metal surface quenches the donor's fluorescence emission and, therefore, decreases the fluorescence lifetime. Since the energy transfer rate depends on the distance between the fluorescent system and the metal surface, the distance value can directly be obtained by converting the fluorescent lifetime. [57] Equivalent to FRET, the metal-induced energy transfer also is a near-field effect. However, the relation between fluorescence lifetime and distance ranges from zero to $100-200 \mathrm{~nm}$ due to the planar geometry of the metal surface.

The phenomenon of the metal-induced energy transfer is mediated by an electromagnetic field, which originates from an oscillating electric dipole. ${ }^{60]}$ Nearly every organic fluorescent dye can be considered as an ideal electric dipole oscillator. The fluorescence lifetime depends on the orientation of the dipole to the metal surface. This dependence is shown in Figure 2.9. [57]

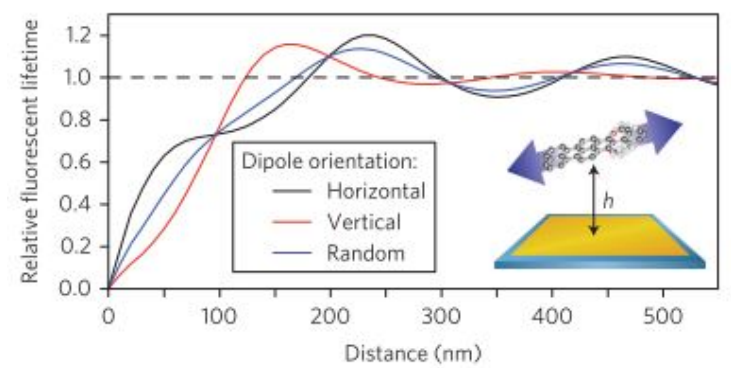

Figure 2.9: Illustration of calculated dependence of the relative fluorescence lifetime to the distance of the fluorescent dye to the metal surface. The fluorescent dye is considered as a oscillating dipole. The orientation of the dipole influences the lifetimedistance dependence. ${ }^{[5]}$ Adapted with permission from A. Chizhik et al., Nature Photonics 2014, 8, and used without modification. 


\section{Chapter 3}

\section{Polymers on surfaces: Let's talk about lifetime}

\subsection{Preface}

The immobilization of polymer chains onto a planar surface dramatically changes their dynamics near the surface when exposed to an external stimulus. Theoretical studies by Müller and Pastorino even predict a reversely driven flow inside a polymer layer. ${ }^{[9]}$ Within these molecular dynamic simulations the polymer layer consists of two brushes: The primary brush is chemically adsorbed to the planar surface, whereas the secondary brush is of shorter chain length and physically adsorbed via an end-functionalization. The weakly adsorbed molecules, therefore, diffuse along the surface. An applied shear flow, with an explicit solvent that does not significantly penetrate into the polymer brush, forces the longer polymer chains to adopt a cyclic motion. This cyclic motion results in a backflow near the surface and a reverse transport of the shorter physically adsorbed molecules.

The experimental investigation of this phenomenon is the main aim of one project within the SFB 937. A novel technique using fluorescence correlation spectroscopy combined with metal-induced energy transfer (MIET) is the tool of choice. Enderlein and co-workers established the MIET method, where a fluorescent system transfers non-radiative energy to the surface plasmons of a metal layer. ${ }^{[57}$ The determination of the fluorescence lifetime makes it possible to localize fluorescent emitters and obtain information about their distance from the metal layer. The combination with fluorescence correlation spectroscopy may then deliver a valuable insight into the vertical and parallel motion of fluorescent molecules that are attached to grafted polymer chains.

Corresponding measurements require a novel synthesis route to produce a homogeneous polymer layer with low glass-transition tempera- 
ture, adjustable grafting density and the possibility to functionalize the polymer with a fluorescent dye. Only surface-initiated reversible additionfragmentation chain transfer (SI-RAFT) polymerization offers the requisite set of properties. The RAFT mechanism allows for polymers with homogeneous chain length. The grafting density can be tailored by specific functionalization of the surface. Subsequent modification of the RAFT group then offers the possibility to attach fluorescent molecules.

Within the first section of this chapter the synthesis of the polymer system is demonstrated. The characteristics of every reaction step needed to be understood to prepare polymer layers with tailored properties. Utilizing the expertise, substrates for MIET measurements were prepared, which are then discussed in the second section of this chapter.

All fluorescence lifetime images with TCSPC curves, MIET curves and the corresponding calculations were done and kindly provided by Jan Christoph Thiele of the Enderlein group.

\subsection{Tuning the process of SI-RAFT polymerization}

\subsubsection{Basic strategy and mechanism}

The investigation of the vertical dynamics of a polymer brush anchored onto a non-adsorbing substrate requires a precise control of the preparation of a defined polymer layer. The average molar mass has to be easily controllable by experimental conditions, for example the concentration of the reactants or the reaction time. Furthermore, the resulting molar mass distribution has to be very narrow and a homogeneous polymer layer with a defined grafting density should be formed. Additionally, it is necessary to incorporate a functional end-group into the polymer chain that can be substituted with a fluorescent dye for spectroscopic measurements.

Polymerization techniques, which provide most of the mentioned controlling characteristics are, for example, reversible-deactivated radical polymerization (RDRP). It provides a polymerization with living characteristics, where the probability of growth of all polymer chains is equal and no transfer or termination reactions take place. ${ }^{[61]}$ The active center of the polymerization remains at the terminal end of the polymer chains, even after the polymerization is completed. With the addition of further monomer molecules and an initiator, the polymerization reaction can be continued. The control of this technique is mainly based on the dynamic equilibrium 
between propagating radicals and dormant polymer chains. A prominent controlling RDRP polymerization technique is the reversible addition-fragmentation chain transfer (RAFT) polymerization. 24,25,27] The introduction of a chain transfer agent that reversibly deactivates propagating radical species provides a controlled character by adjusting the probability for chain growth. A schematic chemical structure of a RAFT agent is shown in Figure 3.1. The main functionalities of a RAFT agent are a thiocarbonyl double bond, a leaving R-group and an activating Z-group. The chemical structure of the R- and Z-group defines the success of the controlled radical polymerization for a specific monomer and experimental conditions.

Within this project a trithiocarbonate was chosen, because the studied monomers represent more activated monomers. [27]

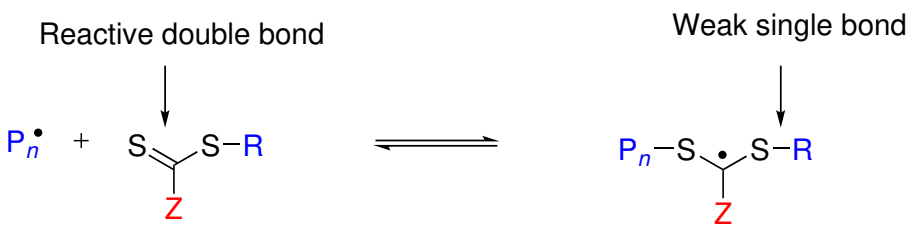

Figure 3.1: Initial equilibrium with pristine RAFT agent and radical species. A typical RAFT agent consists of a thiocarbonyl double bond, a leaving R-group and an activating Z-group.

Within this project, to prepare a polymer layer with covalently attached polymer chains with a defined grafting density, the grafting-from approach was chosen. This method provides the possibility to vary the grafting density from the mushroom regime with a low grafting density to high density polymer brushes. Using this technique the anchoring of the RAFT agent onto the substrate was required. This can be mediated by the R- or the Z-group, the RAFT polymerization is then termed R-group or Z-group approach, respectively. Using the R-group approach the trithiocarbonyl with the Z-group is then located at the $\omega$-terminal end of the polymer chain when the polymerization is completed. This strategy offers the opportunity to incorporate a desired functional end-group by altering the terminal RAFT moiety. To measure fluorescent spectroscopy the chain end needs to be functionalized with a fluorescent dye. 
For the attachment of the RAFT agent via R-group a modification of the surface with a complementary functional group is necessary. Here, the functionalization of a silicon substrate with $\mathrm{NH}_{2}$-terminated ethoxysilane was chosen. The procedure is called surface-initiated (SI) RAFT polymerization.

Figure 3.2 shows the individual steps until the polymerization step. A commercially available silicon substrate was cleaned and activated with air plasma. The resulting hydroxy groups were converted with $\mathrm{NH}_{2}$-terminated ethoxysilane to form a self-assembled monolayer of amino moieties. [62,63] The RAFT agent, which contains a mercaptothiazoline group within the R-group, was anchored to the silicon surface by a substitution reaction with the $\mathrm{NH}_{2}$-groups. SI-RAFT polymerizations with 2-(dimethylamino)ethyl prop-2-enoate (DMAEMA) was conducted afterwards.

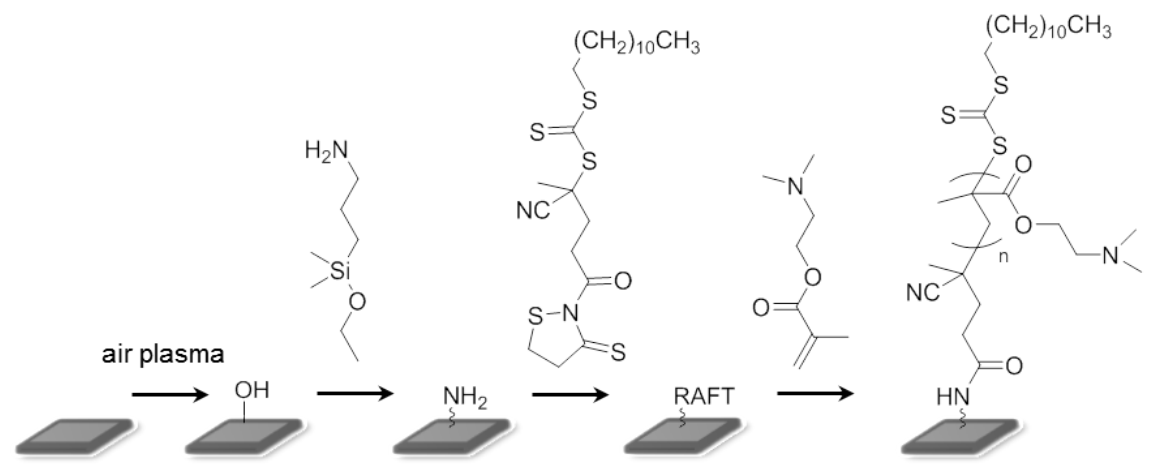

Figure 3.2: Basic strategy of the surface initiated RAFT polymerization of DMAEMA on silicon wafers. Commercially available silicon wafers were treated with air plasma to remove contaminants and form a dense hydroxy group layer. After the reaction with a $\mathrm{NH}_{2}$-terminated ethoxysilane, a monolayer is formed that exhibits $\mathrm{NH}_{2}$-end-groups. These functionalities can react with a mercaptothiazoline-activated RAFT agent. Using the R-group approach DMAEMA can be polymerized on the surface.

In the following sections, the investigation of every step and the corresponding experimental conditions are demonstrated.

\subsubsection{Preparation of ultraflat silicon substrates}

The preparation of chemically adsorbed polymer layers was carried out on $\langle 100\rangle$-oriented silicon substrates. The silicon substrate possesses a native oxide layer when it is stored at ambient conditions. The plasma exposure 
with air allows for the removal of contaminants and generates an $\mathrm{OH}-$ terminated surface. ${ }^{64,65]}$ The $\mathrm{OH}$-terminated surface originates from the exposure to air moisture. The activated surface was extremely hydrophilic with a water contact angle of approximately $\theta<10^{\circ}$ and was stable over several days. Figure 3.3 shows the time stability of the $\mathrm{OH}$-terminated surface after plasma exposure with air. Water contact angle measurements were conducted directly after the treatment and in continuous time intervals until five days. The surface was very slippery, so that the calculation of a water contact angle was hardly possible. The remaining drops at the needle occurred because the substrate was electrically loaded due to the plasma treatment.

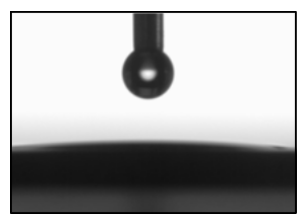

(a) direct

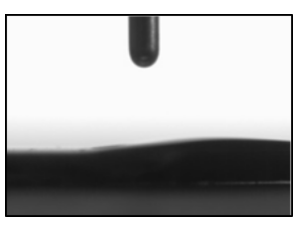

(b) two days

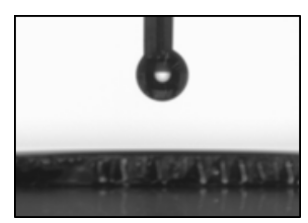

(c) five days

Figure 3.3: Time stability of $\mathrm{OH}$-terminated surface after plasma exposure in air. Water contact angles were measured directly after the treatment, after two and five days. The calculation of the water contact angle was hardly possible and is, therefore, assumed to be $\theta<10^{\circ}$

Although the thickness of the silicon dioxide layer does not influence further reaction steps, it had to be determined, because it is a required parameter to calculate the grafting density of the polymer layer. The grafting density of the polymer layer after the completed SI-RAFT polymerization was calculated from nulling ellipsometry measurements. The height of the formed polymer layer was modeled using the specific optical parameters of the polymer and the polarized light beam reflected from the surface. Therefore, the silicon dioxide layer had to be measured before any further reaction step because the optical parameters are similar to the polymer and the single layer cannot be distinguished afterwards.

Ellipsometric measurements of three different spots of three silicon substrates after plasma exposure were conducted and a homogeneous silicon dioxide layer was obtained that only differed $\pm 0.1 \mathrm{~nm}$ along the substrate. However, the thickness between the three samples showed significant differ- 
ences. Although a homogeneous plasma was used, the thickness depended on the position in the plasma oven. Figure 3.4 shows the distribution of the thicknesses of several substrates with formed silicon dioxide layer after plasma exposure. The thickness differs approximately $\pm 2 \mathrm{~nm}$ within one experiment. Therefore, to calculate the exact grafting density of the resulting polymer layer the thickness of each silicon dioxide layer had to be determined. Within this project it was measured at one spot directly after the plasma exposure.

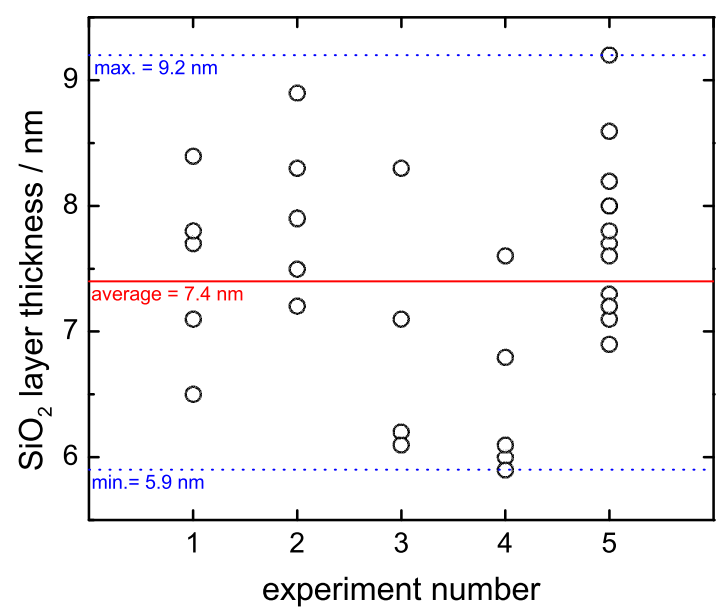

Figure 3.4: Air plasma treatment of a silicon wafer leads to the removal of contaminants and the formation of a silicon dioxide layer. This layer exhibits similar optical properties during ellipsometric measurements compared to a polymer layer. Therefore, the thickness of the silicon dioxide layer needs to be known.

\subsubsection{Tailoring the grafting density}

Within this project three ways to tailor the grafting density were investigated:

- Via grafting-to approach,

- different experimental conditions during silanization,

- usage of a mask during RAFT anchoring. 
Using a grafting-to approach, preformed polymer chains using RAFT polymerization can be attached to a surface modified with a complementary functional group. Here, substrates with a monolayer of amino moieties were used, whereas the RAFT group at the polymer chain end was equipped with a mercaptothiazoline group. Modified silicon substrates were immersed in solutions of poly(methyl acrylate) (PMA) with two different molar masses for time intervals between two and eight hours. The grafting density $\sigma$ in $\mathrm{nm}^{-2}$ was calculated using the following equation: ${ }^{[15]}$

$$
\sigma=\frac{h \cdot \rho \cdot N_{\mathrm{A}}}{\bar{M}_{\mathrm{n}}},
$$

where $h$ is the thickness of the polymer layer, $\rho$ the bulk density of the polymer composition, $N_{\mathrm{A}}$ the Avogadro's number and $\bar{M}_{\mathrm{n}}$ the numberaverage molar mass of the tethered polymer species. Investigated molar masses were $\bar{M}_{\mathrm{n}}=17 \mathrm{~kg} \mathrm{~mol}^{-1}$ and $\bar{M}_{\mathrm{n}}=35 \mathrm{~kg} \mathrm{~mol}^{-1}$ to determine the influence of the chain length on the resulting grafting density. The thickness of the polymer was measured via nulling ellipsometry.

Figure 3.5 shows the dependence between the reaction time and the thickness or grafting density, respectively. For both molar masses, the grafting densities had a small range from $0.05 \mathrm{~nm}^{-2}$ up to $0.10 \mathrm{~nm}^{-2}$. For $\bar{M}_{\mathrm{n}}=17 \mathrm{~kg} \mathrm{~mol}^{-1}$ approximately $20 \%$ higher values for the grafting densities were obtained for every time interval. This tendency is ascribed to the dependence between the redius of gyration and the molar mass. With increasing polymerization degree, the radius of gyration and, thus, the dimension of the polymer chain is increasing. This dependence leads to different grafting densities for different chain lengths at the same experimental conditions.

However, the obtained thicknesses for the PMA layers were larger for the higher molar mass of $\bar{M}_{\mathrm{n}}=35 \mathrm{~kg} \mathrm{~mol}^{-1}$. This observation is also ascribed to the larger radius of gyration compared to $\bar{M}_{\mathrm{n}}=17 \mathrm{~kg} \mathrm{~mol}^{-1}$. The larger polymer coil overlaps with neighboring polymer chains and consequently stretches away from the surface to minimize polymer-polymer interaction.

In Figure 3.6 the transition from non-interacting to highly-interacting regimes of attached polymer chains is illustrated. With increasing grafting density the distance between the attachment points is decreasing. A mushroom regime is present, when the distance is larger then the radius of gyration of the polymer molecule. ${ }^{[15]}$ The chains do not interact and 


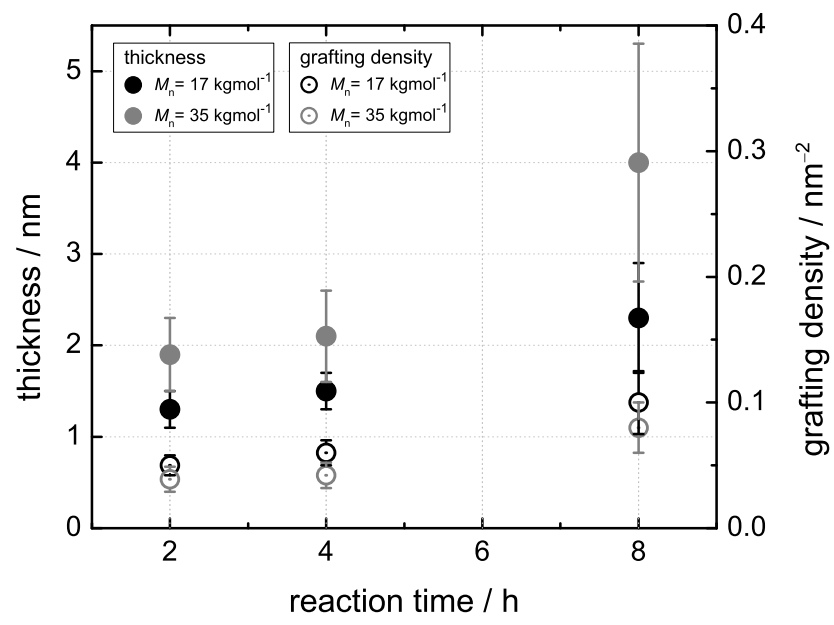

Figure 3.5: Resulting thickness and grafting densities $\sigma$ after different reaction times of grafting-to approach of poly(methyl acrylate) onto amino functionalized silicon substrates. Investigated number-average molar masses were $\bar{M}_{\mathrm{n}}=17 \mathrm{~kg} \mathrm{~mol}^{-1}$ (black) and $\bar{M}_{\mathrm{n}}=35 \mathrm{~kg} \mathrm{~mol}^{-1}$ (grey).

are fully flexible. ${ }^{17]}$ The decrease of the distance leads to a semiflexible regime where chains start to stretch away from the surface to minimize the polymer-polymer interaction. Within the polymer brush regime the chains are highly stretched. Thus, using the grafting-to approach the accessible grafting densities is limited by the steric hindrance of the already attached polymer chains.

Altogether, using the grafting-to approach the grafting density could not be tailored over a wide range. Therefore, further strategies were applied using the grafting-from approach.

It has to be noted, that the investigation of the thickness of the single aminosilane or single RAFT agent layers using spectroscopic ellipsometry was not convincing. The variation of their concentration on the substrate led to thickness differences of maximal $0.5 \mathrm{~nm}$, because the layer thickness with full coverage was also very small and determined to be approximately $2 \mathrm{~nm}$. Water contact angle measurements also showed no significant differences. 


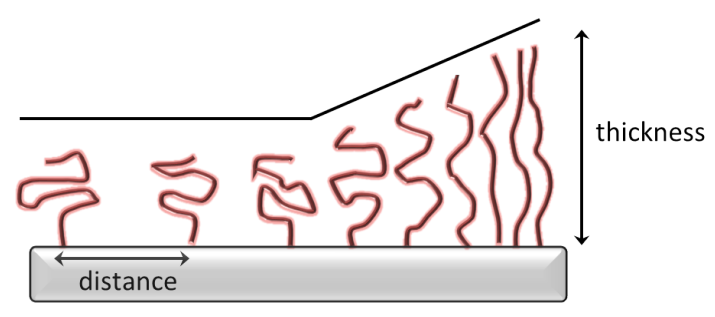

Figure 3.6: Transition from non-interacting mushroom regime to highly-interacting polymer brush. The characteristic parameters thickness and distance between attachment points are also illustrated. A decrease in distance between attachment points forces the polymer chains to stretch away to minimize polymer-polymer interactions. Thus, the polymer layer thickness is increasing.

Consequently, when reaction conditions were altered during, for example, the silanization step or the anchoring of the RAFT agent, all reaction steps including the polymerization had to be conducted. Finally, the resulting polymer layer was studied.

A further strategy to tailor the grafting density was the variation of experimental conditions during the immobilization of aminosilane. Considering the reaction steps shown in Figure 3.2, the number of aminogroups on the substrate limits the number of anchored RAFT agents. Thus, smaller grafting densities are obtained because less polymer chains can be formed on the surface. Parameters that were investigated were the reaction temperature, the reaction time and the concentration of the aminosilane. In Figure 3.7 the dependencies of investigated parameters and the resulting grafting density of polymers are demonstrated.

In Figure 3.7 (a) the influence of the reaction temperature and time is illustrated. The grafting density ranges between $\sigma=0.12 \mathrm{~nm}^{-2}$ and $\sigma=0.23 \mathrm{~nm}^{-2}$. Compared to the grafting-to approach higher values of $\sigma$ were obtained during grafting-from approach. This demonstrates an inherent advantage of the grafting-from approach to the grafting-to process: The steric hindrance of anchored polymer chains hardly influences the propagation. 


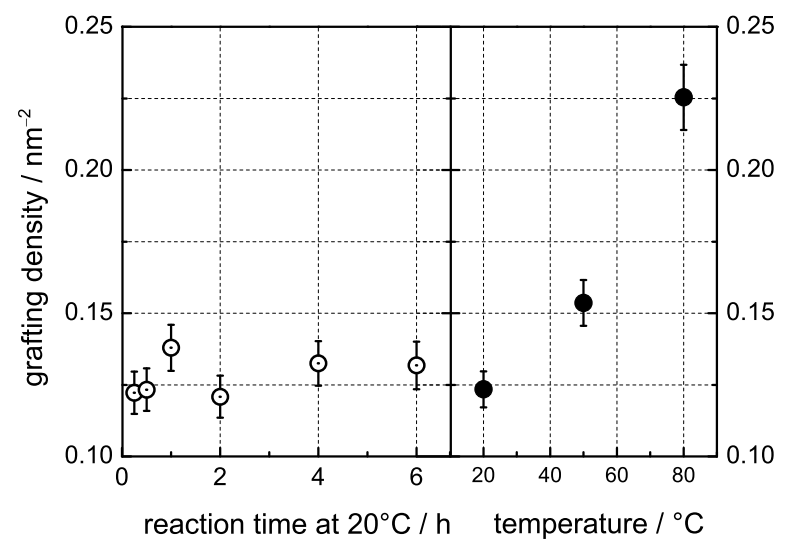

(a)

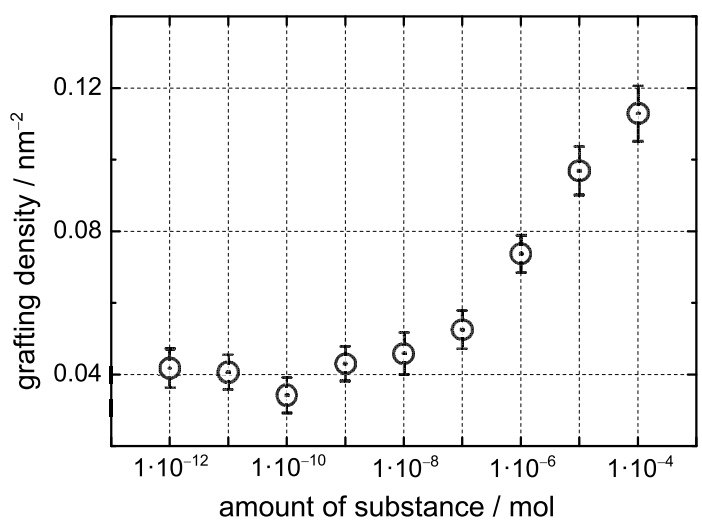

(b)

Figure 3.7: Variation of experimental conditions during silanization of $\mathrm{OH}$-functionalized silicon substrate. In (a) the influence of the reaction temperature and reaction time on the grafting density is shown. Plot in (b) shows the dependence between grafting density and concentration of aminosilane. 
The highest grafting density was obtained at $80^{\circ} \mathrm{C}$ and a reaction time of six hours (see Figure 3.7(a)). Decreasing the reaction temperature from $80{ }^{\circ} \mathrm{C}$ to $20^{\circ} \mathrm{C}$ also led to a decrease of the grafting density from $0.23 \mathrm{~nm}^{-2}$ to $0.13 \mathrm{~nm}^{-2}$.

The influence of the several time intervals to functionalize the silicon dioxide layer with aminogroups at $20{ }^{\circ} \mathrm{C}$ was also investigated. However, the decrease of the functionalization time from six hours to 15 minutes showed no difference. The resulting polymer grafting density remained practically constant at $\sigma=(0.13 \pm 0.01) \mathrm{nm}^{-2}$.

Another tested strategy to form a polymer brush with a low defined grafting density was the variation of the aminosilane concentration (see Figure $3.7(\mathrm{~b})$ ). At a temperature of $20^{\circ} \mathrm{C}$ and functionalization time of three hours, the amount of substance in $1.5 \mathrm{~mL}$ reaction mixture was lowered from $1 \cdot 10^{-4}$ to $1 \cdot 10^{-12} \mathrm{~mol}$. Here, a decrease of the grafting density up to approximately $0.04 \mathrm{~nm}^{-2}$ for $n=1 \cdot 10^{-9}$ mol could be obtained. A lower concentration of aminosilane showed no further difference, so that it remained at $\sigma=(0.038 \pm 0.004) \mathrm{nm}^{-2}$. Even a synthesis route without $\mathrm{NH}_{2}$-functionalization led to a polymer layer thickness of $2.8 \mathrm{~nm}$ that belongs to $\sigma=0.035 \mathrm{~nm}^{-2}$. That means, that also the $\mathrm{OH}$-groups on the silicon substrate could react with the RAFT species in a substitution reaction, which also led to a surface initiated polymerization and a thin polymer layer.

The third strategy to tune the grafting density was the usage of a RAFT mask. This RAFT mask also contains a mercaptothiazoline group that can bind to the silicon substrate via the $\mathrm{NH}_{2}$-moieties. It should occupy active $\mathrm{NH}_{2}$-sites at the substrate and tailor the amount of propagating polymer chains. The chemical structure of the RAFT agent and the corresponding mask is shown in Figure 3.8. Figure 3.8 (a) shows the used trithiocarbonate that is immobilized to the substrate via its R-group and carries a dodecyl group as Z-group on the other side. In Figure 3.8 (b) the immobilized mask is shown. The whole RAFT agent backbone is absent and is only composed of an acetaldehyde group after immobilization.

$\mathrm{NH}_{2}$-functionalized silicon substrates were immersed in solutions with different rates of [RAFT agent]:[RAFT mask] starting from 1:0 to 1:20. However, no differences in the resulting grafting densities were observed after polymerization with 2-(dimethylamino)ethyl methacrylate (DMAEMA). For every ratio, grafting densities of approximately $\sigma=0.2 \mathrm{~nm}^{-2}$ were obtained. During these polymerizations sacrificial RAFT agent was used. That means, a reaction mixture for RAFT polymerization was prepared, consisting of 


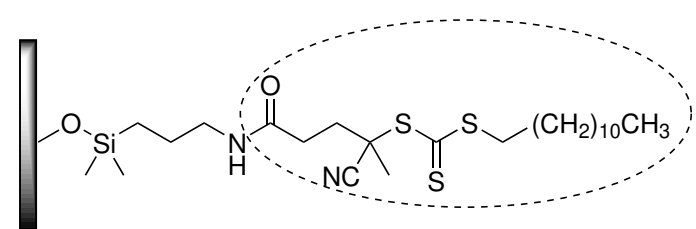

(a) RAFT agent

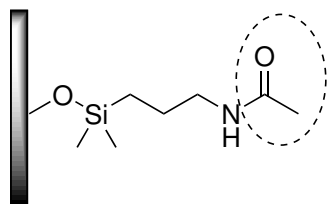

(b) RAFT mask

Figure 3.8: Chemical structures of immobilized RAFT agent and RAFT mask to adjust the grafting density of polymer chains. RAFT mask should react with $\mathrm{NH}_{2}$-moieties and prevents the RAFT anchoring onto the surface.

initiator, RAFT agent and monomer. The amount of immobilized RAFT agent was neglected. This procedure was applied to polymerize free polymer which can be analysed after the reaction is completed. As the RAFT agent, the activated species with incorporated mercaptothiazoline was used. It is termed activated because the mercaptothiazoline group enhances the substitution reaction regarding the yield and the amount of byproducts. [28] During further experiments non-activated RAFT agent was also used as the sacrificial RAFT agent during polymerization. Here, only very low grafting densities between $\sigma=0.03 \mathrm{~nm}^{-2}$ and $\sigma=0.08 \mathrm{~nm}^{-2}$ were obtained.

Figure 3.9 shows the dependence between immobilized layer, the used sacrificial RAFT agent and the resulting grafting density. The uniform bars demonstrate the grafting density using the activated RAFT species, whereas the striped bars represent polymerizations using the non-activated RAFT agent. The substrates above show the respective layers which were applied. Using the activated species the largest grafting densities were obtained. A slight decrease was observed using the $\mathrm{NH}_{2}$-functionalized substrate directly. Similar observations were made for substrates with immobilized RAFT mask. As already mentioned before, the usage of non-activated RAFT agent led to very low grafting densities. Using substrates with immobilized RAFT agent the grafting density decreased from $0.17 \mathrm{~nm}^{-2}$ to $0.08 \mathrm{~nm}^{-2}$. This observation led to the assumption that the formed bond between activated $\mathrm{RAFT}$ agent and $\mathrm{NH}_{2}$-functionalized silicon substrate was not stable during the polymerization of DMAEMA. This assumption is supported by the determination of very low grafting densities of approximately $0.03 \mathrm{~nm}^{-2}$ using non-functionalized substrates and non-activated RAFT agent. However, the formation of large grafting densities using non-functionalized substrates and activated RAFT agent during the polymerization was an 


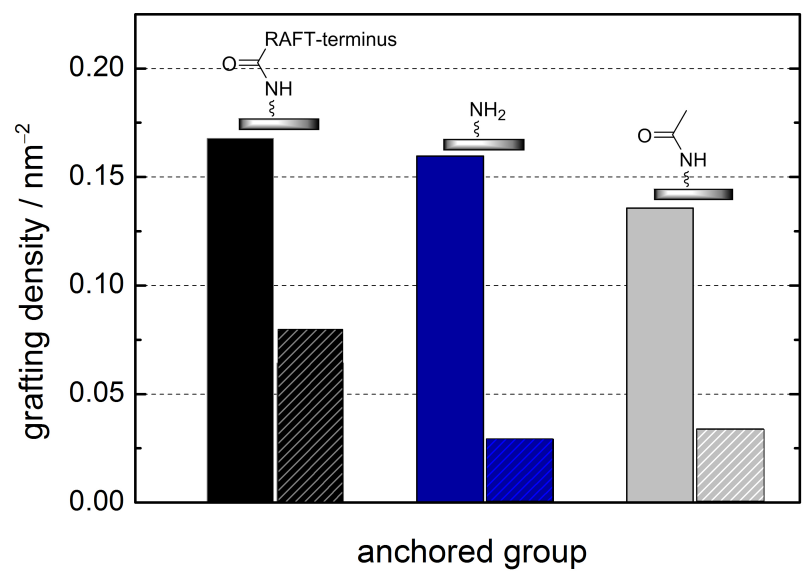

Figure 3.9: Summary of resulting grafting densities using differently functionalized substrates. The adjustment of the polymer grafting density was investigated using the RAFT agent, an anchored RAFT mask and a $\mathrm{NH}_{2}$ layer. Uniform bars represent polymerization reactions with the activated RAFT agent as a sacrificial controlling agent, whereas the striped bars represent reactions with the non-activated RAFT agent.

evidence for coupling reactions of $\mathrm{NH}_{2}$-groups and activated RAFT or polymer species during polymerization reactions.

The reason for this observation could be the formed peptide bond during immobilization. From literature it is known that the decomposition rate of peptide bonds increases with increasing temperature. ${ }^{[66]}$ Additionally, this process could be catalysed by bases - DMAEMA could potentially act as a base in this instance. The polymerization of DMAEMA was conducted at $90{ }^{\circ} \mathrm{C}$ for several hours. This could lead to the partial decomposition of the peptide bond, so that a steady exchange between substrate and polymerization solution occurred. This would lead to a combined mechanism of grafting-to and grafting-from approach. Using the grafting-to approach polymer grafting densities up to $0.1 \mathrm{~nm}^{-2}$ were obtained. From literature it is known, that the largest grafting density is observed using the grafting-from approach. ${ }^{[28]}$ For instance, a RAFT polymerization of methyl methacrylate on silica nanoparticles yielded a grafting density of approx- 
imately $0.3 \mathrm{~nm}^{-2}$. The largest value determined within this project was intermediate at $0.23 \mathrm{~nm}^{-2}$. This observation also leads to the assumption of a combined mechanism of surface initiated polymerization and the grafting of propagating polymer chains onto the surface.

To summarize, it was demonstrated that the grafting density of polymer immobilized on planar silicon substrates could be varied deliberately between $0.03 \mathrm{~nm}^{-2}$ and $0.23 \mathrm{~nm}^{-2}$. It appears reasonable to assume that the obtained range covers weakly and strongly interacting polymer chains. Therefore, it is expected that a different impact on the vertical diffusion in fluorescence lifetime correlation spectroscopy would result.

\subsubsection{RAFT polymerization of DMAEMA}

In Section 3.2.3 the necessity to use the activated RAFT agent as a sacrificial RAFT agent during polymerization was demonstrated. Using the activated species guarantees a grafted polymer layer onto silicon substrates with a large grafting density.

To investigate the effect of the R-group on the RAFT polymerization, polymerizations of DMAEMA mediated by the non-activated and activated RAFT agent were carried out. In Figure 3.10 the chemical structure of the investigated RAFT agents are shown. Figure 3.10 (a) shows the non-activated structure of 4-cyano-4-[(dodecylsulfanyl-thiocarbonyl)sulfanyl]pentanoic acid (CDSPA) whereas in Figure 3.10 (b) the activated species with mercaptothiazoline moiety is illustrated. The difference between these molecules simply is the substitution of the $\mathrm{OH}$-group with mercaptothiazoline within the R-group of the RAFT agent.<smiles>CCCCCCCCCCCCCCCCCCC(C)(C#N)SC(O)=S</smiles>

(a) CDSPA

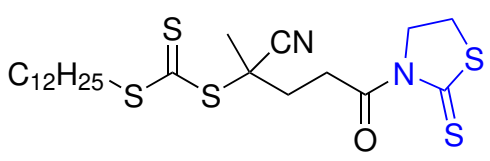

(b) activated CDSPA

Figure 3.10: Chemical structures of non-activated RAFT agent (CDSPA) and mercaptothiazoline-functionalized RAFT agent (activated CDSPA). The blue marked functionalities indicate the different moieties within the R-group, which is investigated concerning the influence on the polymerization step. 
During the RAFT mechanism propagating radicals are adding to the controlling agent via the reactive thiocarbonyl double bond. ${ }^{[24]}$ The R-group can then be cleaved homolytically. The rate of fragmentation is mainly influenced by the nature of the R-group:

- Steric effects,

- electron-withdrawing groups and

- radical stabilizing groups on the R-group.

The selection of the R-group depends on the applied monomer species. For instance, RAFT agents with $\mathrm{R}=\mathrm{CH}_{2} \mathrm{Ph}$, such as benzyl dithiobenzoate, serve as suitable leaving groups for the polymerization of styryl and acrylyl propagating radicals. However, the benzyl group is a poor chain transfer agent for the polymerization methacrylyl radicals. It has to be noted that the impression, that the R-group should be chemically constructed as the monomeric species, is misleading. The penultimate unit effect can lead to a poorly controlled polymerization: The R-group is a poor leaving group for macromolecular propagating radicals, although the R-group would be a suitable chain transfer agent for the monomeric radicals. ${ }^{[67,68 \mid}$ A similar effect is attributed to sterically demanding substituents at the R-group. ${ }^{68]}$

The chain transfer agent does not only have to contain a suitable leaving group regarding the monomer, but the formed radical $\mathrm{R} \bullet$ has to be able to initiate a polymerization reaction with a monomer and form a further propagating radical. ${ }^{[24]} \mathrm{A}$ triphenylmethyl radical serves as a perfect leaving group, but the rate of re-initiation would be too low. In this case, the chain transfer agent would lead to a retardation of the RAFT polymerization.

The best strategy to estimate the effectiveness of a RAFT agent for specific polymerization systems is to conduct experiments under the required conditions. The evolution of the obtained molar mass over time and the dispersity of the molar mass distribution have to be evaluated in order to determine if a RAFT polymerization was successful. ${ }^{271}$

The RAFT polymerization of DMAEMA was performed under similar experimental conditions as used for the surface initiated polymerization. Under exclusion of oxygen DMAEMA was polymerized at $90^{\circ} \mathrm{C}$ using $1,1^{\prime}-$ azobis(cyclohexanecarbonitrile) (ACCN) with dimethylformamide (v/v=0.2) and the respective RAFT agent.

In Figure 3.11 the size-exclusion chromatograms obtained from polymerization with CDSPA (picture (a)) and activated CDSPA (picture (b)) 
are shown. Using a UV detector, which measures the absorption in the ultraviolet (UV) range, the presence of RAFT group per polymer chain is detected. [69] From the refractive index signal (RI) of the obtained polymer, the molar mass can be calculated. In both cases the size-exclusion chromatograms from the UV detector overlap with the RI signal. Thus, both RAFT species are suitable chain transfer agents for the polymerization of DMAEMA. The controlled character of the RAFT polymerization is also demonstrated by pseudo-first-order plots of the obtained number-average molar mass after several time intervals (see Figure 3.12). The molar mass increased linearly with reaction time. However, the polymerization mediated by the activated CDSPA appears to have larger polymerization rates than with CDSPA. Even after a polymerization time of 60 minutes the number-average molar mass $\bar{M}_{\mathrm{n}}$ differs by $25 \%$ from $14.9 \mathrm{~kg} \mathrm{~mol}^{-1}$ to $19.9 \mathrm{~kg} \mathrm{~mol}^{-1}$. After a reaction time of 240 minutes a number-average molar mass of $44.2 \mathrm{~kg} \mathrm{~mol}^{-1}$ was obtained for the non-activated RAFT agent, whereas a number-average molar mass of $63.8 \mathrm{~kg} \mathrm{~mol}^{-1}$ was obtained for activated CDSPA. This corresponds to a difference of approximately $30 \%$. For the corresponding dispersities of the molar mass distributions no significant difference were observed. For both polymerization systems the main RAFT equilibrium seems to be established after a longer polymerization time, as can be concluded from the decrease in dispersity over time.

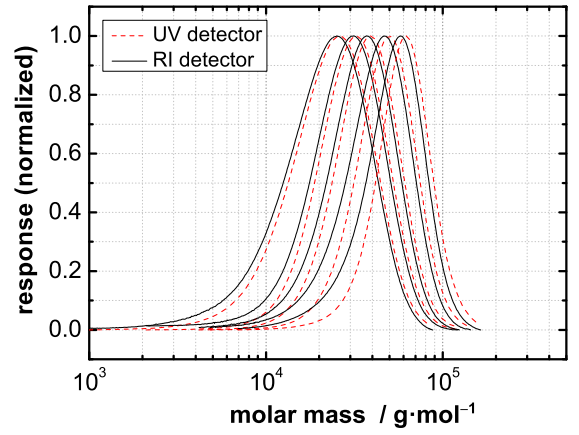

(a)

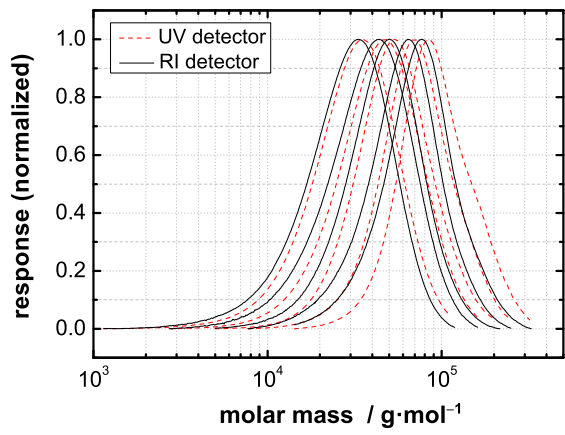

(b)

Figure 3.11: (a) and (b) show the size-exclusion chromatograms of the RAFT polymerization of DMAEMA using the non-activated RAFT agent CDSPA and the activated CDSPA species, respectively. Black distributions result from a refractive index detector. The red dotted lines were recorded using a UV detector detecting the RAFT group. 


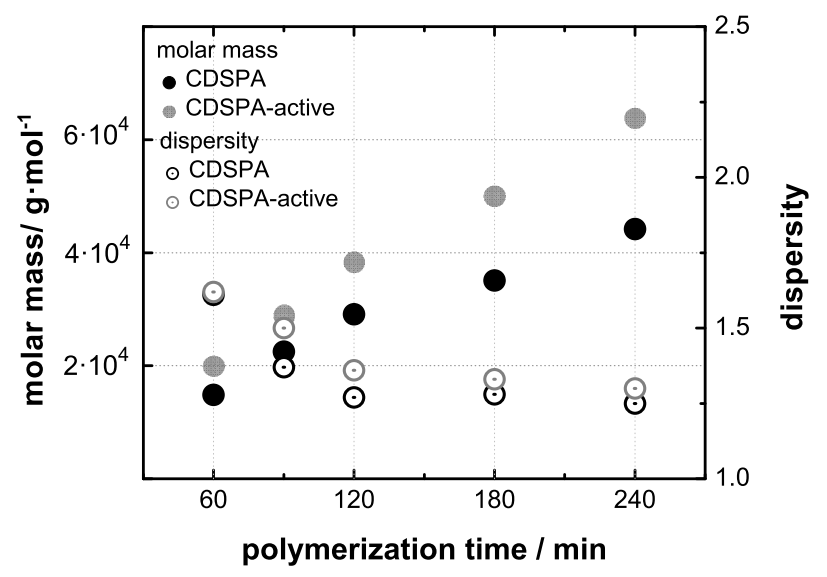

Figure 3.12: Comparison of RAFT polymerization of DMAEMA with non-activated RAFT agent (CDSPA) and activated RAFT agent (CDSPA-active). It shows the obtained number-average molar mass and corresponding dispersity after several time intervals mediated by the different RAFT species.

\subsubsection{SI-RAFT polymerization of DMAEMA}

In order to check if the results of the polymerization in solution are transferable to surface modification corresponding experiments had to be conducted. The main aim of these experiments was to determine the influence of the obtained mechanism of combined grafting-to and grafting-from processes mentioned in Section 3.2.3.

Silicon wafer were modified with a different amount of aminosilane to yield two different grafting densities of DMAEMA (see Section 3.2.3). Five $\mathrm{NH}_{2}$-functionalized substrates of each grafting density were separately immersed in a polymerization solution of monomer, RAFT agent and radical initiator for a defined time interval. The polymerization mixture was quenched at the same time in order to compare the molar mass of the polymer in solution with the one of the grafted polymer chain on the substrate. Figure 3.13 illustrates the determined thicknesses and grafting densities for different number-average molar masses which were obtained for different polymerization times. For both batches of differently dense $\mathrm{NH}_{2}$-functionalized substrates the thicknesses of the polymer layer were increasing linearly with the increase of the number-average molar mass of 
the polymer in solution. Therefore, no significant change of grafting density with increasing molar mass can be obtained. Even for long chains with $\bar{M}_{\mathrm{n}} \approx 80 \mathrm{~kg} \mathrm{~mol}^{-1}$ the same grafting densities as for low molar masses could be produced. In comparison to substrate immobilization using the grafting-to approach, where the grafting density decreases with the increase of molar mass, the combination of grafting-from and grafting-to processes during SI-RAFT polymerization had no influence on the resulting grafting density.

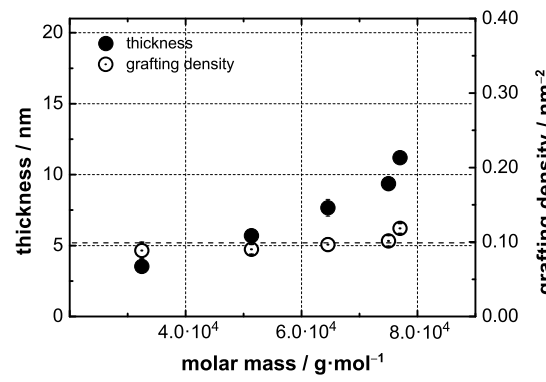

(a)

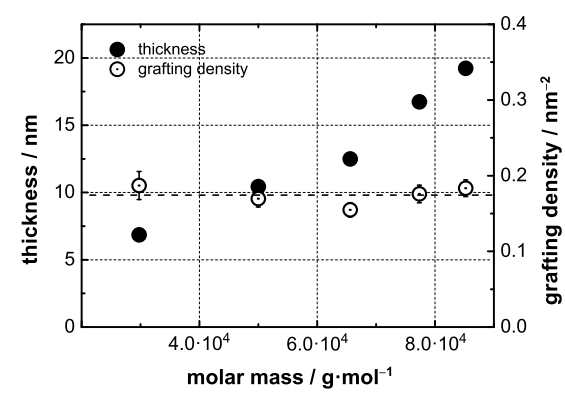

(b)

Figure 3.13: Resulting thicknesses and grafting densities of DMAEMA layer with increasing number-average molar masses. The difference in grafting density was achieved by the variation in number of $\mathrm{NH}_{2}$-groups on the silicon substrate.

In addition to the quantitative analysis via spectroscopic ellipsometry, water contact angle (WCA) measurements were performed to have a clear evidence in surface modification. ${ }^{70]}$ Therefore, the wettability properties were measured after each reaction step: $\mathrm{NH}_{2}$-functionalization, immobilization of the RAFT agent and surface-initiated polymerization with DMAEMA. Figure 3.14 illustrates the determined water contact angles after each reaction step. The different values for the respective surface modifications were obtained by multiple experiments over different samples to check the reproducibility. For the $\mathrm{NH}_{2}$-functionalized surface a WCA of $\theta=(71 \pm 2)^{\circ}$ was obtained. Referring to literature, the obtained WCAs for the $\mathrm{NH}_{2}$-terminated ethoxysilane indicate a less hydrophilic surface. ${ }^{[71,72]}$ It is assumed that this is the result of residual solvent molecules. The functionalization was conducted in different organic solvents (dioxane, tetrahydrofuran and toluene) and yielded different WCAs each time. 
However, the successful surface modification was proved by the change in wettability. The subsequent modifications with CDSPA and p(DMAEMA) also showed significantly different WCAs and can thus be considered successful. Within these modifications no change in WCA was observed which is a result of the R-group approach. After polymerization the Z-groups of the RAFT agent form the outer layer.

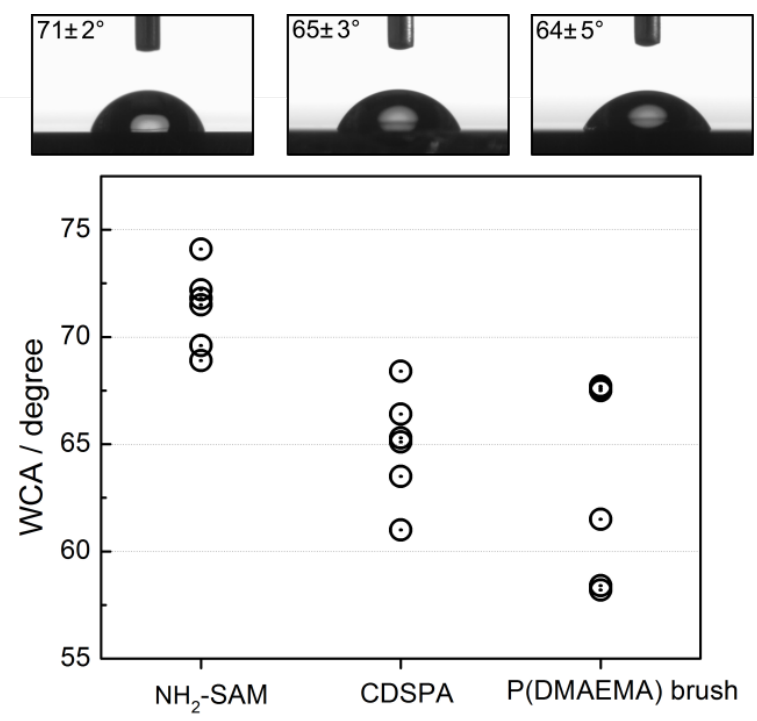

Figure 3.14: Water contact angle (WCA) measurements can be performed to qualitatively analyze the modification of a surface. The obtained WCA after every reaction step are illustrated. The different values for the respective surface modifications were obtained by multiple experiments over different samples to check the reproducibility.

\subsection{Fluorescence microscopy}

Within this chapter investigations using fluorescence microscopy of polymer chains tethered onto a planar substrate will be presented. As mentioned in the introduction, these studies were part of the SFB 937, in which the vertical and horizontal motion of fluorescent molecules attached to grafted polymer chains should be investigated. Ultimately, understanding these model systems should yield valuable insight into the dynamics of biological systems, such as the endothelial glycocalyx layer. ${ }^{[73,74]}$ Biological systems 
are highly sophisticated in their structure and function. Synthetic polymers are excellent candidates to mimic such systems. In order to resemble the tailored functions of biopolymers, p(DMAEMA) was selected as model system due to its $\mathrm{pH}$ responsiveness. Additionally, $\mathrm{p}$ (DMAEMA) exhibits a glass transition temperature below room temperature. ${ }^{75]}$ This is necessary in order to observe the cyclic motion of tethered polymer chains, which is a combination of vertical and horizontal motion. 9

To characterize the components of a cyclic motion, the combination of FCS and MIET appeared to be promising as Enderlein and co-workers introduced a novel concept to localize individual fluorescent molecules and perform FCS measurements on diffused systems. ${ }^{[60,76]}$ Therefore, the scope of the application of these techniques on fluorescently labelled p(DMAEMA) chains on surfaces should be explored. The model system was investigated in the dry state and in aqueous solution with $\mathrm{pH}=7$ and $\mathrm{pH}=2$. In Figure 3.15 the expected conformations in different environments of tethered polymer chains are shown in accordance with theoretical considerations given previously, see Section 2.1.2.
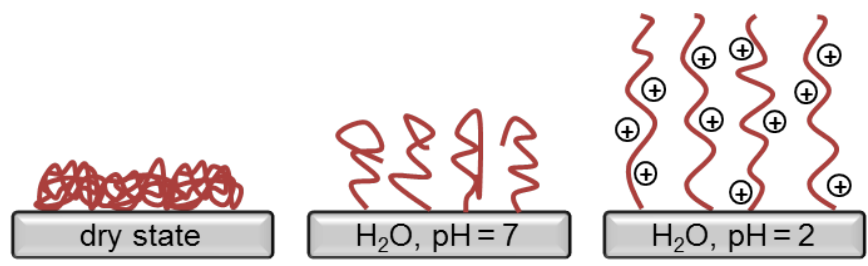

Figure 3.15: Expected conformations of tethered $\mathrm{pH}$-responsive $\mathrm{p}(\mathrm{DMAEMA})$ polymer chains in the dry state and in aqueous solution at different $\mathrm{pH}$ value. In the dry state a uniform polymer layer with a low thickness value is formed. Exposed to water, the polymer chains swell and the thickness increases. An aqueous solution at $\mathrm{pH}=2$ forces the chains to stretch because of repulsive interactions.

Polymer layers with different grafting densities and different molar masses were synthesized using surface-initiated RAFT polymerization. At the $\omega$-terminal end of the polymer chains, a fluorescent dye was incorporated. Metal-induced energy transfer was used to establish a relation of the fluorescence lifetime and the thickness of the polymer layer. In order to evaluate the results obtained from MIET measurements, spectroscopic ellipsometry was also conducted for polymer layers in the dry state and in aqueous solution at $\mathrm{pH}=7$. Furthermore, an autocorrelation analysis 
was conducted to investigate the fluctuation of the fluorescently labelled polymer layer (see Closing remarks and outlook).

\subsubsection{Fundamentals}

For the application of fluorescence microscopy of single molecules suitable substrates were required. The preparation of substrates with a homogeneous polymer layer was conducted using the strategies mentioned in the previous chapter. First, the substrate was functionalized with $\mathrm{NH}_{2}$-moieties, which provide the complementary substitution functionality to the activated RAFT group. Via RAFT polymerization a defined polymer layer was immobilized onto the substrate. To equip the tethered polymer chain with a fluorescent dye at the $\omega$-terminal end, the RAFT group was cleaved via aminolysis. The obtained SH-group was then used to attach AтTO655 functionalized with a maleimide moiety.

Substrates with different grafting densities, which vary from weakly to strongly interacting regimes, were achieved by different temperatures and concentration of aminosilane during the functionalization with $\mathrm{NH}_{2}$ moieties. For further information regarding this strategy, the reader is referred to Chapter 3.2.3.

Within this project $150 \mu \mathrm{m}$ thick glass cover slides of $(2.4 \times 2.4) \mathrm{cm}$ were used and covered with the required layer via evaporation deposition. For the immobilization of a defined polymer layer, where the substrate is immersed in the polymerization mixture, a suitable reaction flask was required. The size of the substrate required a flask with an appropriate diameter, which allows for carefully placing and taking out the substrate. Homogeneous reaction conditions were mandatory, however, mixing had to occur without contact of the stirring device to the substrate. All desired properties were met by a custom-made flask shown in Figure 3.16 .

The fluorescently labelled polymer layers were measured using a custombuilt confocal microscope equipped with a device for time-correlated single photon counting (TCSPC) and fluorescence lifetime measurements. In a typical experiment, scan images with a size of $80 \mu \mathrm{m} \times 80 \mu \mathrm{m}$ were acquired to determine the homogeneity of the prepared sample. From the taken scan, four smaller images with $30 \mu \mathrm{m} \times 30 \mu \mathrm{m}$ were scanned when aggregates were absent. If not otherwise mentioned, a pixel size of $100 \mathrm{~nm}$ was selected with a pixel dwell time of $1 \mathrm{~ms}$ and a TCSPC resolution of 16 ps. Figure 3.17 shows fluorescence intensity images of grafted DMAEMA with a molar mass of $\bar{M}_{\mathrm{n}}=36 \mathrm{~kg} \mathrm{~mol}^{-1}$ and a grafting density of $\sigma=0.11 \mathrm{~nm}^{-2}$. 


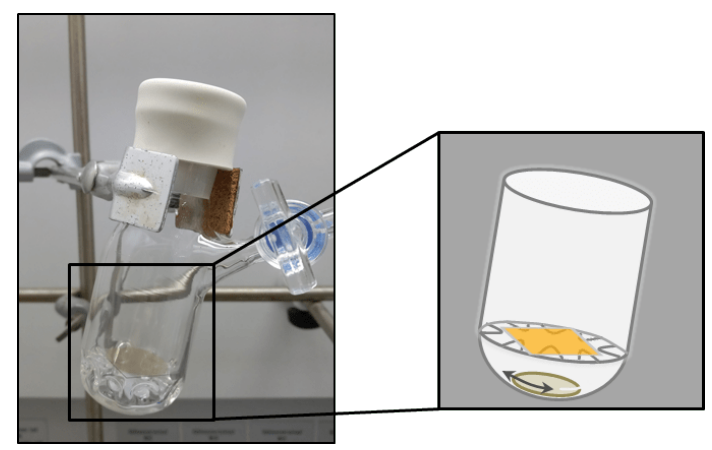

Figure 3.16: The size of the substrate required an appropriate amount of the reaction mixture. Thus, a custom-built flask was needed to retain the controlling RAFT polymerization properties.

The polymer layer was grafted onto a glass substrate, which was covered with a thin gold layer of $10 \mathrm{~nm}$ and a silicon dioxide spacer of $30 \mathrm{~nm}$. The gold layer is incorporated into two thin titanium layers that serve as adhesion layers. ${ }^{777}$ In the scan image on the right the intensity of single molecules can be observed. The background also shows fluorescent activity that can be ascribed to the photoluminescence of gold. 60 The excitation of a smooth gold surface with light of the visible spectrum lead to the emission of photons with an average arrival time of approximately 2 ps. ${ }^{\text {.78] }}$ Therefore, to calculate fluorescence lifetimes which can only be attributed to the fluorescent dye, a cut-off of $0.5 \mathrm{~ns}$ was taken into account.

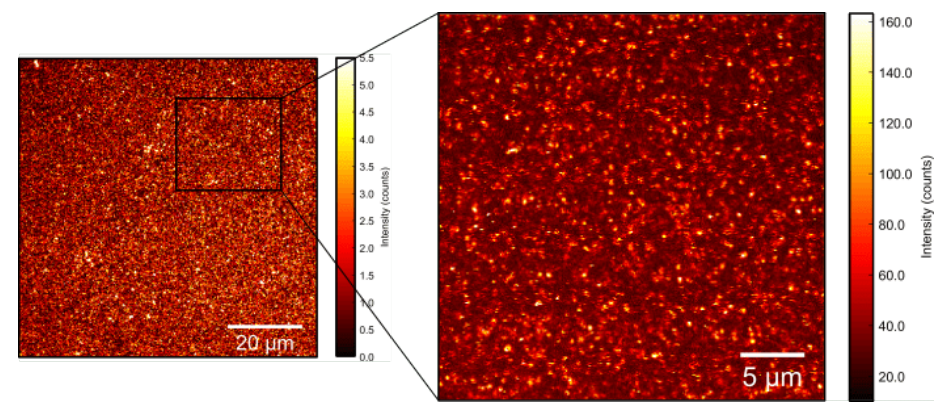

Figure 3.17: Fluorescence intensity scan image showing single molecules grafted onto a glass substrate. From scans of $80 \mu \mathrm{m} \times 80 \mu \mathrm{m}$ (left), four scans of $30 \mu \mathrm{m} \times 30 \mu \mathrm{m}$ were taken. 


\subsubsection{MIET measurements}

Within this project a novel concept to determine the distance between a fluorescent molecule and a metal surface is used. It is based on the energy transfer of the optically excited molecule to the metal surface plasmons. ${ }^{[57]}$ This process is termed metal-induced energy transfer (MIET).

The measurement of the fluorescence lifetime yielded distance values via a theoretical model, which is exemplarily illustrated in Figure 3.18. Here, the relation between the fluorescence lifetime of Aтт0655 in water and the distance to a $10 \mathrm{~nm}$ thick gold layer is shown. The model curve initially shows a linear increase and levels off at approximately $70 \mathrm{~nm}$. A $30 \mathrm{~nm}$ thick silicon dioxide layer (see picture (b) in Figure 3.18) was deposited on the gold layer in order to reduce lifetime measurement errors at small distances. It is pointed out, that the shown theoretical model is exemplary for measurements in water. Distances of fluorescently labelled polymer layers in a dry state or other solvents require corresponding model curves, respectively.

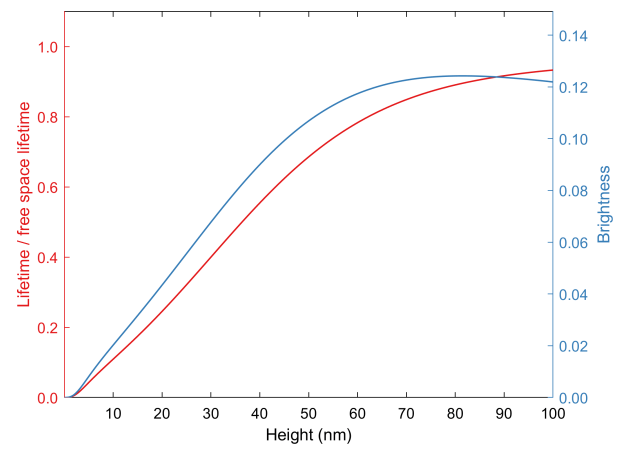

(a)

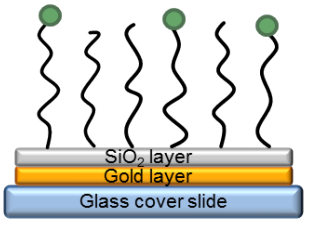

(b)

Figure 3.18: Picture (a) shows a theoretical model of Aтто655 in water and the distance via height to a $10 \mathrm{~nm}$ thick gold layer. Picture (b) illustrates a typical sample preparation with end-labelled polymer chains that are grafted onto a silicon dioxide spacer.

In Figure 3.19 fluorescence lifetime images (FLIM) of a dry p(DMAEMA) layers with a molar mass of $\bar{M}_{\mathrm{n}}=36 \mathrm{~kg} \mathrm{~mol}^{-1}$ and a grafting density of $\sigma=0.11 \mathrm{~nm}^{-2}$ are shown. In Figure 3.19 (a) the FLIM scan of a fluorescently labelled $\mathrm{p}$ (DMAEMA) layer on a glass substrate with silicon dioxide spacer 
is shown. The measurements on glass substrates in absence of a metal layer were conducted in order to establish a reference model for each sample.

In Figure 3.19 (a) a multitude of lifetimes was observed. The fit of the corresponding TCSPC curve was based on the maximum likelihood estimator and is shown on the right. ${ }^{[79]}$ It yielded three components with a large absolute deviation. An average lifetime of the two dominant components of $\tau=2.69$ ns was determined. In comparison, Figure 3.19 (b) illustrates the metal-induced energy transfer of the additional gold layer. Here, fluorescence lifetimes were distributed more homogeneously over the surface. This is also reflected by the three components calculated from the TCSPC curve. The average lifetime decreased to $\tau=0.76$ ns due to the lifetime quenching of the thin gold layer.
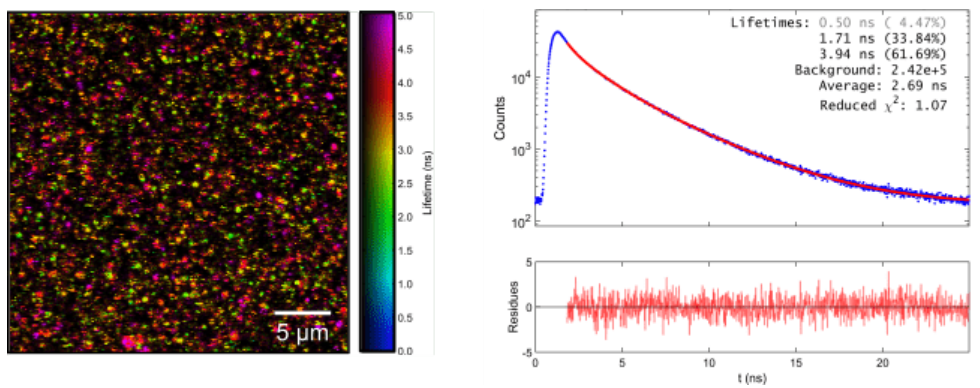

(a) FLIM measurement and fitted TCSPC curve on glass
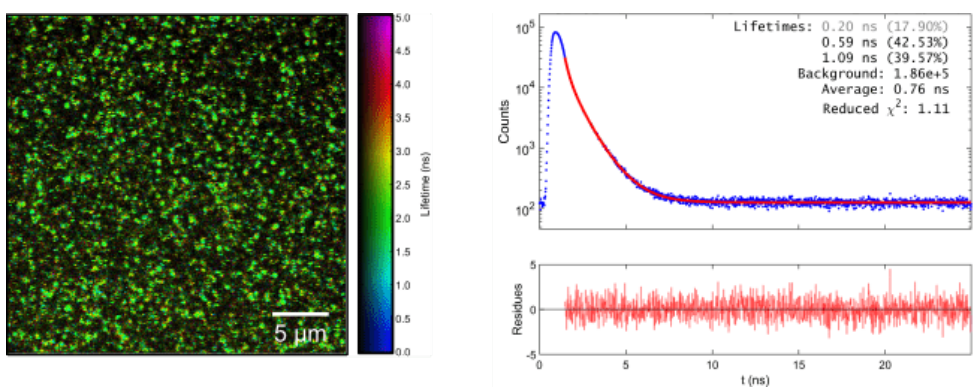

(b) MIET measurement on $\mathrm{Au}$

Figure 3.19: Fluorescence lifetime images of a p(DMAEMA) layer with a molar mass of $\bar{M}_{\mathrm{n}}=36 \mathrm{~kg} \mathrm{~mol}^{-1}$ and a grafting density $\sigma=0.11 \mathrm{~nm}^{-2}$. The curves at the right represent TCSPC curves that were used to obtain the average fluorescence lifetimes. 
Applying MIET the fluorescence lifetime could be converted into the thickness of the polymer layer using a theoretical model which describes the chain end distribution of a polymer brush. ${ }^{80]}$ As explained in the introductory part of this chapter, different grafting densities and molar masses were investigated. The grafting density ranged from $\sigma=0.1 \mathrm{~nm}^{-2}$ to $\sigma=0.4 \mathrm{~nm}^{-2}$. For each grafting density, polymers with two different molar masses were prepared (see Table A1 in the Appendix A). Each sample was measured in three different environments. Figure 3.20 shows representative fluorescence lifetime images of the higher molar mass polymer at three different grafting densities in the dry state. Corresponding fitted TCSPC curves are also shown for each sample.

With increasing grafting density from Figure 3.20 (a) to (c) the fluorescence label density increases significantly. For the lowest grafting density single molecule signals were obtained, whereas for higher grafting densities very dense regions of fluorescence emission were observed. The visual appearance did not change for measurements in aqueous solution at $\mathrm{pH}=7$ and $\mathrm{pH}=2$. From TCSPC curves average fluorescence lifetimes could be calculated, which are increasing from $0.8 \mathrm{~ns}$ for the low grafting density to $1.4 \mathrm{~ns}$ for the highest grafting density. This indicates an increasing average distance of the fluorophores to the metal surface. Such an increase is expected because the higher grafting density forces the polymer chains to adopt more stretched conformations. This also agrees well with the experimental results for $\mathrm{p}$ (DMAEMA) on silicon substrates shown in Figure 3.13 .

Table 3.1 summarizes the calculated thicknesses for all samples in the different environments. In addition, the thicknesses for dry polymer layers determined via ellipsometric measurements are listed. In Table A1 the number-average molar masses, dispersities and grafting densities of all samples are collected. For lower grafting densities the thicknesses could not always be obtained from lifetime measurements. It is assumed that the number of photon counts compared to the backround was too small. At higher grafting densities the polymer thickness in all environments could be calculated. As already shown in Figure 3.15 it is expected that the thickness increases when the polymer chains are swollen in aqueous solution. The polymer chains should also stretch the most at $\mathrm{pH}=2$ due to the electrostatic repulsion. These tendencies were indeed observed using MIET measurements. 

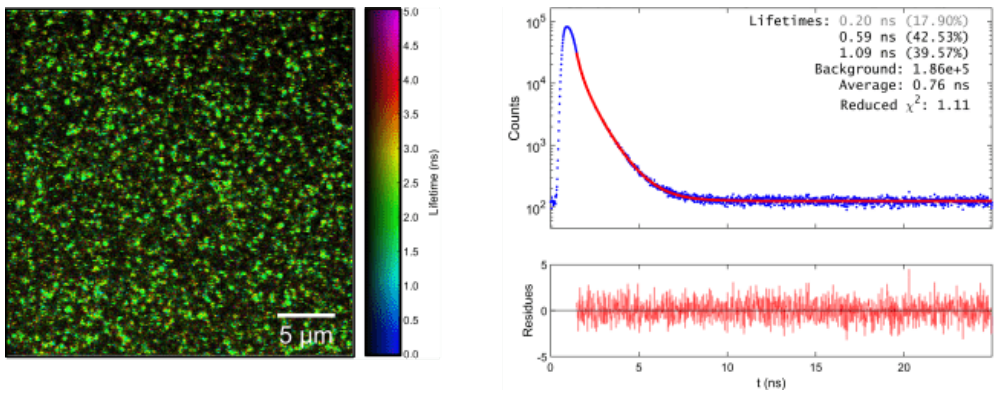

(a) low grafting density
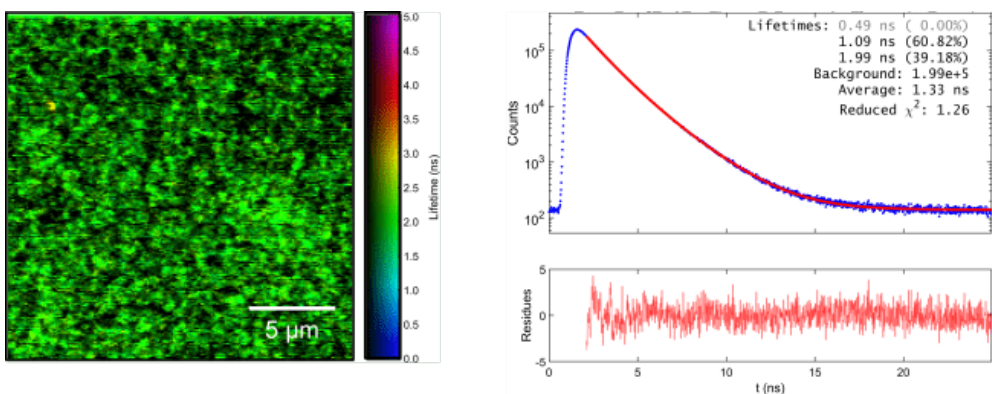

(b) medium grafting density
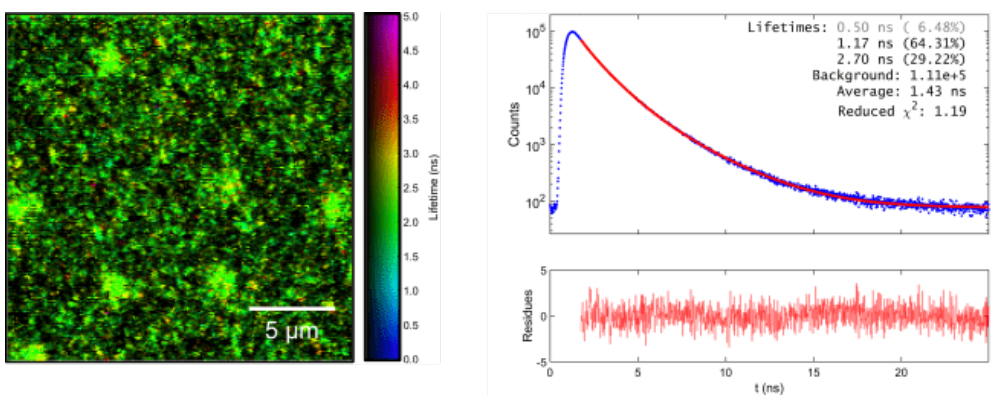

(c) high grafting density

Figure 3.20: Fluorescence lifetime images of a $\mathrm{p}$ (DMAEMA) layer with different grafting densities of $0.1 \mathrm{~nm}^{-2}, 0.2 \mathrm{~nm}^{-2}$ and $0.3 \mathrm{~nm}^{-2}$. The number-average molar mass was $\bar{M}_{\mathrm{n}}=36 \mathrm{~kg} \mathrm{~mol}^{-1}$ for each sample. The curves at the right represent fitted TCSPC curves that were used to obtain the average fluorescence lifetimes. 
Table 3.1: Summary of calculated thicknesses, which were determined via spectroscopic ellipsometry $h_{\text {ellips }}$ and MIET measurements $h_{\text {MIET }}$. The abbreviations LD, MD and HD represent samples with a low, medium and high grafting density, respectively (see Table A1). The indices $l$ and $h$ indicate lower and higher number-average molar masses. The numbers in brackets represent the thickness ratio of polymer chains in aqueous solution at $\mathrm{pH}=2$ and in the dry state, i.e. the swelling ratio.

\begin{tabular}{llcccccc}
\hline & & $\mathrm{LD}_{1}$ & $\mathrm{LD}_{\mathrm{h}}$ & $\mathrm{MD}_{1}$ & $\mathrm{MD}_{\mathrm{h}}$ & $\mathrm{HD}_{1}$ & $\mathrm{HD}_{\mathrm{h}}$ \\
\hline \hline & dry & - & 2.4 & 1.6 & 5.5 & 10.3 & 10.0 \\
$h_{\text {MIET }} / \mathrm{nm}$ & $\mathrm{pH}_{7}$ & - & - & - & 20.6 & 40.6 & 43.5 \\
& $\mathrm{pH}_{2}$ & - & $6.3(2.6)$ & 1.8 & $22.8(4.1)$ & 49.2 & $74.8(7.5)$ \\
\hline$h_{\text {ellips }} / \mathrm{nm}$ & dry & 1.4 & 4.8 & 5.2 & 9.5 & 12.0 & 14.0 \\
\hline
\end{tabular}

If only the higher molar mass samples are considered, comparing the thicknesses in the dry state and in aqueous solution at $\mathrm{pH}=2$ unveils the tendency of an increasing swelling ratio with increasing grafting density (see Table 3.1). Due to the grafting density the polymer chains may interact weakly or strongly with each other, as already explained in the theoretical Chapter 2.1.1. Exposed to aqueous solution at $\mathrm{pH}=2$ the polymer chains are positively charged, causing electrostatic repulsion. Therefore, at low grafting densities only the intramolecular segment-segment interaction increases and forces the polymer chains to adopt stretched conformations. With increasing grafting density the distance of grafting points decreases and also polymer-polymer interactions of neighboring polymer chains become more pronounced. Consequently, the chains stretch even further.

Considering the samples $\mathrm{HD}_{1}$ and $\mathrm{HD}_{\mathrm{h}}$ swollen in aqueous solution at $\mathrm{pH}=2$ one finds a factor of 1.52 between the thicknesses. This agrees well with the ratio of number-average molar masses between the two samples which is 1.44 (see Table A1). Comparing the thicknesses in aqueous solution at $\mathrm{pH}=7$, the change is significantly less pronounced. This is in accordance with the work of Thomas et al. ${ }^{[1]}$ who found a larger swelling factor for $\mathrm{p}$ (DMAEMA) in aqueous solution at $\mathrm{pH}=2$ compared to $\mathrm{pH}=7$. It can be easily understood in terms of the contribution of electrostatic repulsive forces described in the previous paragraph.

In order to discuss the thicknesses obtained via MIET measurements, spectroscopic ellipsometry was applied in the dry state. These measurements were done after the MIET measurements to prevent the fluorescent 
system from bleaching, since wavelengths corresponding to the absorption wavelength of the fluorescence dye were used. Compared to ellipsometric measurements in the dry state the values showed deviations, especially for low and medium grafting densities. There are several possible reasons to explain this observation.

The determination of the thicknesses via ellipsometry may lead to higher values because contaminants can remain at the surface after a sequence of MIET measurements. Also, the thicknesses of the metal layers and silicon dioxide spacer have to be considered for both measurements. Via ellipsometry the composition of the substrate and the thickness of each layer was determined individually. The obtained values showed only slight deviations for the metal layer, but wide variations for the silicon dioxide spacer compared to the target thicknesses. During vapor deposition of each layer the thickness was monitored by using an oscillating quartz unit. The target thickness was $30 \mathrm{~nm}$ for the silicon dioxide spacer, but was determined to be $h=22.8 \mathrm{~nm}$ via ellipsometry. Applying this value for the models used by the ellipsometer yielded the polymer layer thicknesses $h_{\text {ellips }}$ shown in Table 3.1. However, the use of $22.8 \mathrm{~nm}$ for the height of the silicon dioxide layer in calculations from MIET measurements did not lead to physically meaningful values for the thicknesses. Therefore, the target thickness of $30 \mathrm{~nm}$ was used in the MIET calculations to convert the fluorescence lifetime into a thickness.

Ellipsometric measurements take into account all polymer chains of a sample cross-section, whereas MIET only considers the chains that are fluorescently labelled. For MIET it cannot be ensured that the average lifetime is representative for the whole sample due to the inherent molar mass distribution of the polymer. It can be envisioned that, for example, longer polymer chains are predominantly labelled since their end-groups are more exposed to the labelling solution. Though the polymer functionalization was conducted using RAFT, which is a controlled polymerization technique, dispersities of 1.3 to 1.4 were obtained. Consequently, this may also have a significant impact on the determined average lifetimes.

Nonetheless, general tendencies of ellipsometer and MIET measurements are in agreement. It was also possible to investigate samples of $\mathrm{p}$ (DMAEMA) with a number-average molar mass of approximately $\bar{M}_{\mathrm{n}}=80 \mathrm{~kg} \mathrm{~mol}^{-1}$ and different grafting densities in aqueous solution at $\mathrm{pH}=7$ via ellipsometry. The results are shown in Figure 3.21. Both measurements yielded the same slope for the increase of thickness with the grafting density in aqueous 
solution. Though this is not an exhaustive proof, it improves the confidence in the obtained results.

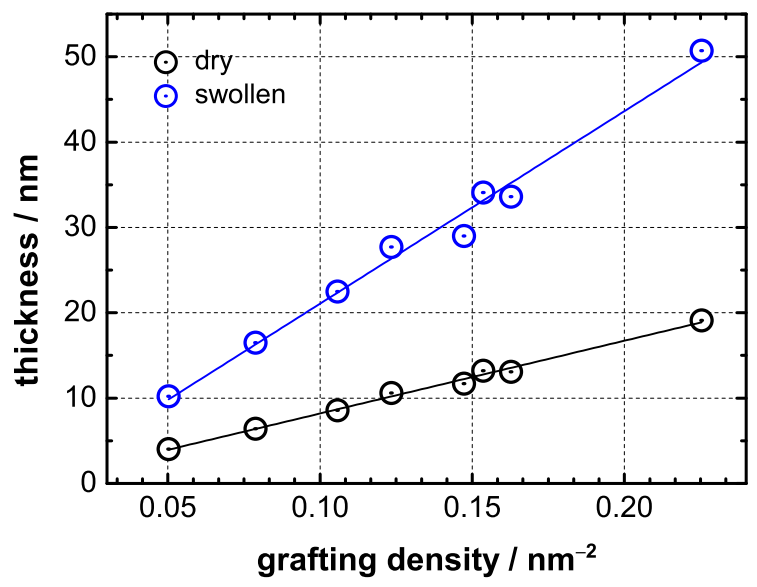

Figure 3.21: Ellipsometric determination of polymer layer thicknesses in dry state and swollen in water at $\mathrm{pH}=7$. Measured samples were P(DMAEMA) with $\bar{M}_{\mathrm{n}}=$ $80 \mathrm{~kg} \mathrm{~mol}^{-1}$ at different grafting densities.

\subsection{Closing remarks to Chapter 3}

In the last chapter the preparation of polymers with tailored chain lengths and grafting densities on planar surfaces was demonstrated. Surfaceinitiated RAFT polymerization was extensively investigated to develop strategies to conduct RAFT polymerization on substrates which could be used to perform MIET measurements.

Preliminary studies on silicon substrates showed that the grafting-to approach is limited to small grafting densities due to the steric effect of already grafted polymer chains. Only the application of the grafting-from technique delivered tunable grafting densities in a range of interest. In addition, the molar masses could be easily tailored using RAFT polymerization. The formed polymer layers could be qualitative analyzed by water contact angle measurements, which proved the successful reactions on the surface by changing wettability properties. A quantitative characterization was performed by spectroscopic ellipsometry. The construction of adjusted 
model systems within ellipsometric calculations led to the possibility to measure polymer thicknesses of just a few nanometers.

In order to perform fluorescence spectroscopy the results from the preliminary studies of surface-initiated RAFT polymerization had to be transferred to glass cover slides, which were covered with gold and silicon dioxide. These substrates differed in size and reactivity compared to the previously used silicon substrates. A new set-up to synthesize polymer layers had to be established to yield equal polymerization conditions. Due to a larger reaction chamber the exact number-average molar masses could not be perfectly reproduced. Additionally, the molar mass distributions increased when the polymerization reaction took place in the new set-up to polymerize on glass cover slides. However, the control over the grafting density could be retained and was tuned from $0.1 \mathrm{~nm}^{-2}$ to $0.4 \mathrm{~nm}^{-2}$. For each regime of tethered polymer the length of the polymer chains was varied from low to high molar masses.

After labelling with a fluorescent dye the prepared polymer layer of different grafting densities could be investigated using fluorescence lifetime imaging. From fitted TCSPC curves fluorescence lifetimes were calculated and converted into polymer layer thicknesses using a chain end distribution model. Thicknesses varied with the polymer grafting density in accordance with theoretical descriptions of surface-tethered polymers. Interesting tendencies of the thicknesses of $\mathrm{p}$ (DMAEMA) swollen in aqueous solution at $\mathrm{pH}=7$ and $\mathrm{pH}=2$ were obtained. At $\mathrm{pH}=2$ the influence of electrostatic repulsion within the chains and between neighboring polymer chains could be identified.

The results presented in this chapter should be considered as a starting point for future studies. It was proved that MIET can be utilized successfully with fluorescently labelled synthetic polymer chains. The evaluation of results also revealed the high complexity of the topic which requires even more intensive interdisciplinary collaboration in order to address all parameters. Several potential improvements were presented and discussed. 


\section{Chapter 4}

\section{Bringing structure into the world of polymers}

\subsection{Preface}

"Small is beautiful." [82] This tender title of a scientific article and the abundance of publications concerning nano- and microscience illustrates the fascination of the scientific world to synthesize and study species on the nanometer scale. [83] "There's plenty of room at the bottom." was the title of the famous lecture held by Richard P. Feynman in 1959 in which he clarified his visions to manipulate individual atoms. [82]

These days it is possible to create structures at a nanometer scale using electron-beam-induced deposition and produce computer chips which are $7 \mathrm{~nm}$ small. ${ }^{[84,85]}$ Creating structured surfaces is one of the major tasks in micro- and nanotechnology. ${ }^{[8]}$ Here, polymeric systems can be a smart tool to tune surface properties, for instance permeability, roughness, wettability or biocompatibility. [87]

Numerous approaches to tailor nanostructured polymer surfaces were recently established. ${ }^{[8]}$ Using electron beam lithography feature sizes down to $70 \mathrm{~nm}$ can be generated. [86,89] Dip-pen nanolithography, nanoshaving and scanning electrochemical microscope etching are further examples for the fabrication of polymeric nanopatterned surfaces. $90-92$

A major advantage of using polymer layers is their immediate response to external stimuli such as temperature, solvent quality or light. [18,93] The response to external stimuli often leads to a conformational change within the polymeric layer. Examples are block-copolymer brushes, which can undergo conformational change by microphase separation due to its immiscible polymer blocks under certain experimental conditions. ${ }^{94,95]}$ Thermoresponsive polymers, for instance, show an adaptive behaviour to different temperatures and collapse or swell at critical solution temperatures. ${ }^{[88]}$ 
The combination of temperature and different $\mathrm{pH}$ value forces a blockcopolymer of a thermoresponsive and a $\mathrm{pH}$-responsive block to rearrange in a preferential segregation depending on the experimental conditions. ${ }^{966}$

The morphology transition cannot just be induced by different temperatures and $\mathrm{pH}$, but also by altering solvent quality. For instance, a two component mixed brush with one hydrophilic and one hydrophobic homopolymer segregates if the brush is exposed to a hydrophilic solvent. [97] The hydrophilic homopolymer forms the top of the brush and the surface becomes hydrophilic. The surface can be switched into a hydrophobic polymer layer when the brush is exposed to a hydrophobic solvent. The mixed polymer brush adopts the thermodynamic property of the current solvent.

In the present work, structured polymer layers with features of several nanometers were obtained using only an individual homopolymer. Polystyrene was anchored onto a non-adsorbing substrate with a low reduced grafting density and exposed to constrained dewetting. As a consequence polymer chains collapse onto the substrate to minimize polymer-solvent interaction. ${ }^{35,36]}$ The formed polymer aggregates constitute a polymeric nanostructure with different types of possible nanopatterns, which are shown in Figure 4.1. The morphology of the nanostructure mainly depends on the reduced grafting density, but does the polymer topology also influence the type of morphology?

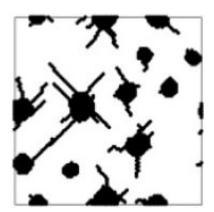

(a)

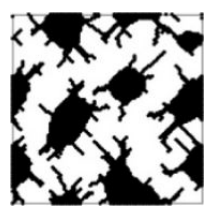

(b)

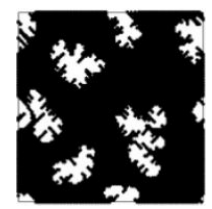

(c)

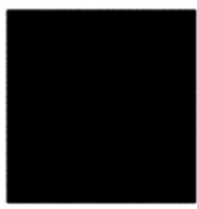

(d)

Figure 4.1: Simulated nanostructures mediated by constrained dewetting of grafted polymer onto a non-adsorbing surface. Increasing grafting density from (a) to (d) yields different morphologies. 36

To adress this question polystyrene was synthesized via reversible additionfragmentation chain transfer (RAFT) polymerization. This controlled polymerization technique provides the possibility to control end-group functionality and polymer topology. ${ }^{[98]}$ The thiocarbonylthio group of the RAFT 


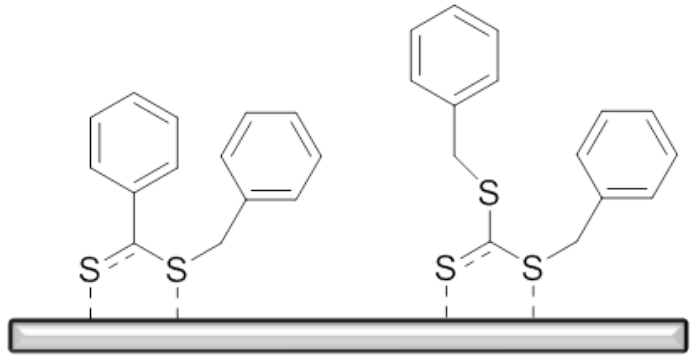

(a)

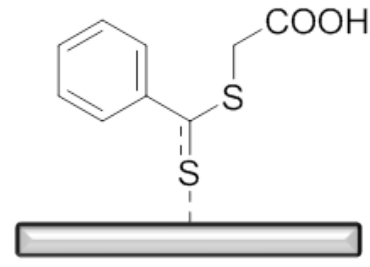

(b)

Figure 4.2: Binding modes of RAFT agents on gold surfaces. (a) Shows a possible configuration proposed by Duwez et al, whereas in (b) monodentate binding proposed by Blakey et al is illustrated. $100[101$

moiety was already introduced as an anchoring group to flat gold surfaces and gold nanoparticles. ${ }^{[99-101]}$ Figure 4.2 illustrates two possible configurations proposed in literature. [100,101] A recent publication demonstrates the successful anchoring of a thiol terminated polymer chain onto gold substrates. ${ }^{[102]}$ Considering these investigations, it is still unknown, if a polymeric system can be immobilized onto a flat gold surface solely via its containing RAFT moiety. To answer this question and prove the presence of polymer on the gold surface, atomic force microscopy and water contact angle measurements were conducted within this project.

As already mentioned, the RAFT polymerization technique also provides the opportunity to synthesize defined polymer topologies. For example, branched macromolecules can be achieved by a RAFT agent with star topology. 99,103 Employing the R-group approach, each arm of the star polymer contains a RAFT functionality at the terminal end that can function as an anchoring group.

Using the constrained dewetting methodology provides the possibility to further functionalize the uncovered regions on the substrate. Especially new ways for the preparation of noble metallic nanostructures, such as gold and silver, are demanded. ${ }^{[104]}$ The most interesting characteristic of these nanostructured metal surfaces is the enhanced absorption in the ultraviolet and visible spectral regions. ${ }^{[05]}$ This phenomenon is exploited in surface enhanced Raman spectroscopy, an application to identify and characterize a wide range of substances. Therefore, investigations on the assembly of gold nanoparticles on polymer functionalized substrates were conducted. 
Two questions, which had to be answered, were:

- Can colloidal clusters of nanoparticles within a polymeric nanostructure be generated?

- Can the stimuli-responsive characteristics of the polymer layer still be retained?

This would be highly attractive for multifunctional, stimuli-responsive surfaces.

\subsection{Fundamentals}

In the following section, the substrate preparation and preliminary studies on the formation of nanostructues via constrained dewetting are introduced.

\subsubsection{Preparation of ultraflat metal surfaces}

The substrate has to meet suitable conditions for the immobilization of the polymer layer and the formation of structures with nanometer dimensions. Therefore, a suitable substrate needs to be:

- clean and active enough to bind sulphur atoms,

- ultraflat to not influence the morphology of the nanopattern.

There are several possibilities to prepare appropriate gold surfaces. Many substrates with different qualities in their roughness are commercially available, however, sufficiently flat substrates have a very high cost. Furthermore, there are three common possibilities to prepare suitable substrates oneself. One method is template stripping (TS). ${ }^{106 l}$ After depositing the gold layer on a very smooth mica substrate, the gold film is glued upside-down on to a mechanical support. The mica substrate is then stripped off to yield a flat metal surface. Another method to prepare suitable substrates is sputtering. ${ }^{107]}$ Here solid gold is bombarded with highly energetic particles so that surface atoms are emitted into the gas phase, followed by their deposition on the substrate. This method results in a low roughness but often suffers from the poor adhesion of the gold particles to the substrate. This disadvantage can be circumvented by the deposition of a gold layer by evaporation. ${ }^{[108]}$ Before the evaporation of a gold nanolayer a thin adhesion 


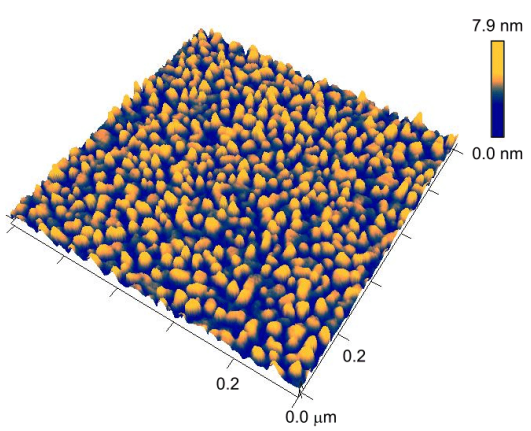

(a)

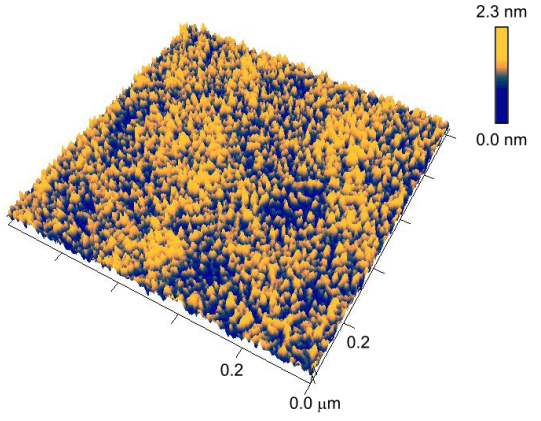

(b)

Figure 4.3: AFM images of glass substrates evaporated with different thicknesses of gold. Image (a) shows a gold layer with a thickness of $50 \mathrm{~nm}$ that forms a globular structures. Image (b) depicts a $10 \mathrm{~nm}$ thick gold layer with high homogeneity.

layer can be deposited on the substrate. Hereby, atomically flat surfaces over extended areas are achieved.

In this work, glass substrates with titanium as an adhesion layer and subsequently a thin gold nanolayer were used. The glass substrates were cleaned thoroughly to serve as a suitable template for ultraflat surfaces (see Section 6.2). A commonly used literature parameter to quantify the roughness of a surface is the root mean squared roughness $R_{\mathrm{q}}$ that can be calculated via the image analysis software NanoScope Analyis. The purification of the glass substrates yielded a very low roughness of $R_{\mathrm{q}}=0.24 \mathrm{~nm}$ over a $3 \times 3 \mu \mathrm{m}$ image.

Such ultraflat substrates are essential for the vapor deposition of atomically flat metal surfaces. During the evaporation process of the adhesion layer and subsequent gold film, there are several important parameters that have a great impact on the morphology of the gold nanolayer. [108] First, the deposition of an ultraflat metal film is mainly dependent on the thickness of the evaporated layer. It also depends on how fast the metal is transferred into the gas phase, called evaporation rate, and the temperature during the vapor deposition.

Figure 4.3 shows two images recorded by atomic force microsocopy (AFM) that exhibits glass cover slides evaporated with $2 \mathrm{~nm}$ titanium as an adhesion layer and different thicknesses of the gold nanolayer. In Figure 4.3 
(a) the gold layer has a thickness of $50 \mathrm{~nm}$ whereas in (b) a representative image of a $10 \mathrm{~nm}$ thin gold film is shown. Both samples were deposited at ultrahigh vacuum, room temperature and an evaporation rate of $1 \AA \mathrm{s}^{-1}$. The increase of the thickness of the evaporated gold led to a higher surface roughness. The root mean square roughness started from $R_{\mathrm{q}}=0.33 \mathrm{~nm}$ for $10 \mathrm{~nm}$ thin gold layer and raised to $R_{\mathrm{q}}=1.15 \mathrm{~nm}$ when the thickness was increased to $50 \mathrm{~nm}$. A globular gold structure appeared as it is shown in Figure 4.3. ${ }^{[108]}$ The variation of the evaporation rate was studied as well and yielded very low values for the root mean sqaure roughness for rates up to $3 \AA \mathrm{s}^{-1}$. The best conditions to yield an ultraflat metal surface for the imaging of polymeric structures with nanometer dimensions were very thin gold layers up to $10 \mathrm{~nm}$ deposited with an evaporation rate of $1 \AA \mathrm{s}^{-1}$.

Surface cleanliness of a fresh evaporated gold nanolayer was studied by water contact angle (WCA) measurements. In numerous publications the nature of clean gold and its hydrophilicity is discussed controversially. [109] The WCA of clean gold ranges from $10^{\circ}$ to $80^{\circ}$. The freshly prepared gold substrates used in this study had a contact angle of $\theta=80^{\circ}$. To clean and activate metal surfaces plasma treatment is used very often. ${ }^{[102]}$ Hence, the influence of air plasma on the surface morphology was investigated accordingly.

After a short treatment of $30 \mathrm{~s}$ with $50 \mathrm{~W}$ air plasma a hydrophilic gold surface with a water contact angle of $\theta=35^{\circ}$ was recorded. However, this activation treatment led to a very high surface roughness with an extreme change of its morphology. Figure 4.4 shows the AFM images of two gold substrates with and without air plasma cleaning. Additionally the water contact angle of both surfaces are illustrated. At the left side a freshly prepared gold substrate without further purification is shown. The right images demonstrates the influence of a short air plasma treatment. Although the contact angle decreases to $35^{\circ}$, the AFM image shows sharp, elevated patterns and the homogeneity of the metal surface is destroyed. During air plasma treatment the temperature of the substrates is increasing because of the high amount of applied energy. A possible explanation for the observed structural change can therefore be the expansion of the metal surfaces. 


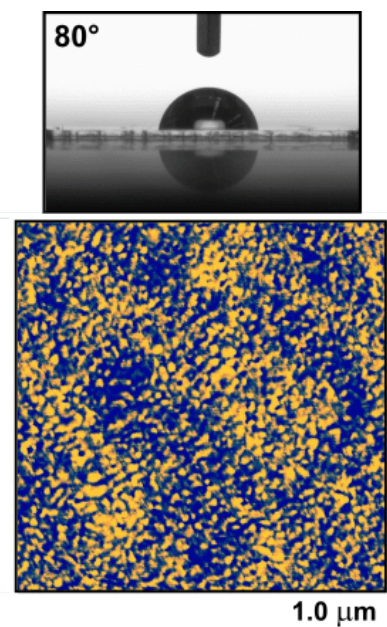

(a) before plasma treatment

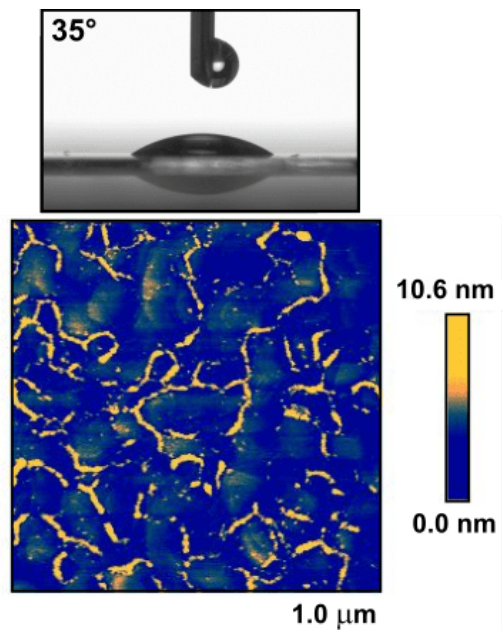

(b) after plasma treatment

Figure 4.4: Surface properties of evaporated gold nanolayer on glass substrates before and after air plasma treatment.

\subsubsection{Characterization of thin polystyrene layers}

The morphology of a nanostructured surface formed by constrained dewetting of a polymer layer depends on the grafting density of the tethered polymer. ${ }^{[35,36]}$ A commonly used literature parameter to define a thin polymer layer is the grafting density $\sigma$ in grafted chains per $\mathrm{nm}^{2}$. [15] It is determined as follows:

$$
\sigma=\frac{h \cdot \rho \cdot N_{\mathrm{A}}}{\bar{M}_{\mathrm{n}}}
$$

where $h$ is the thickness of the polymer layer, $\rho$ the bulk density of the polymer composition, $N_{\mathrm{A}}$ the Avogadro's number and $\bar{M}_{\mathrm{n}}$ the number averaged molar mass of the tethered polymer.

The distance $D$ between the grafting points of immobilized polymer chains can be derived from the grafting density $\sigma:[18]$

$$
D=\sigma^{-(1 / 2)} \text {. }
$$


To compare different architectures of immobilized polymer molecules the reduced grafting density $\Sigma$ is a more specific parameter to describe how much space a polymer molecule occupies on the substrate. ${ }^{[15]}$ A linear polymer chain with a particular molar mass takes up a different amount of space than a star shaped polymer with the same molar mass. The reduced grafting density $\Sigma$ is proportional to the root mean square radius of gyration $\left\langle s^{2}\right\rangle^{1 / 2}$ that describes the dimensions of a polymer molecule under specific conditions like temperature and solvent. Thus $\Sigma$ is defined as the number of macromolecules that occupy a defined area on the surface compared to the area that a free not interacting polymer chain employs at the same experimental conditions. The reduced grafting density is related to the grafting density $\sigma$ as follows: ${ }^{[18]}$

$$
\Sigma=\sigma \cdot \pi \cdot\left(\left\langle s^{2}\right\rangle^{1 / 2}\right)^{2}
$$

The radius of gyration for linear polystyrene chains is literature known and determined as $\left\langle s^{2}\right\rangle_{\operatorname{lin}}^{1 / 2}=1.23 \cdot 10^{-2} \cdot \bar{M}_{\mathrm{n}}^{0.5936}$ in toluene at $25^{\circ} \mathrm{C}$. [110] For branched polymer architectures the radius of gyration $\left\langle s^{2}\right\rangle_{\text {br }}$ can be calculated from $\left\langle s^{2}\right\rangle_{\text {lin }}$ using the classical Zimm-Stockmayer prediction: [111,112]

$$
\left(\frac{\left\langle s^{2}\right\rangle_{\mathrm{br}}}{\left\langle s^{2}\right\rangle_{\text {lin }}}\right)_{M}=g_{\mathrm{s}}=\frac{3 f-2}{f^{2}}
$$

with the contraction factor $g_{\mathrm{s}}$ and the number of monodisperse arms $f$ of the branched polymer.

The obtained reduced grafting densities for linear and four-arm star polystyrene with different molar masses are summarized in Figure 4.5. The thickness of the immobilized polystyrene layer was determined by nulling ellipsometry when a homogeneous polymer layer was present. Ellipsometry revealed thicknesses up to $5 \mathrm{~nm}$ for linear polystyrene with $\bar{M}_{\mathrm{n}}=22 \mathrm{~kg} \mathrm{~mol}^{-1}$. For $\bar{M}_{\mathrm{n}}=40 \mathrm{~kg} \mathrm{~mol}^{-1}$ and $\bar{M}_{\mathrm{n}}=60 \mathrm{~kg} \mathrm{~mol}^{-1}$ layer thicknesses were obtained to be $2 \mathrm{~nm}$ at maximum. Thus, polymer layers made out of polystyrene with $\bar{M}_{\mathrm{n}}=22 \mathrm{~kg} \mathrm{~mol}^{-1}$ yielded the highest possible reduced grafting densities $\Sigma$ whereas layers with $\bar{M}_{\mathrm{n}}=60 \mathrm{~kg} \mathrm{~mol}^{-1}$ resulted in the lowest possible reduced grafting densities $\Sigma$.

Since the gold substrate is a non-adsorbing surface the reason for this tendency is connected to the behaviour of a polymer chain diluted in a 
solvent. As already mentioned above the radius of gyration can be used to describe the dimensions of a macromolecule. Because of the increase of the degree of polymerization, the radius of gyration of the polymer chain is also increasing. Larger polymer chains occupy more space and therefore, for similar reduced grafting densities, larger distances are observed for increasing molar masses.

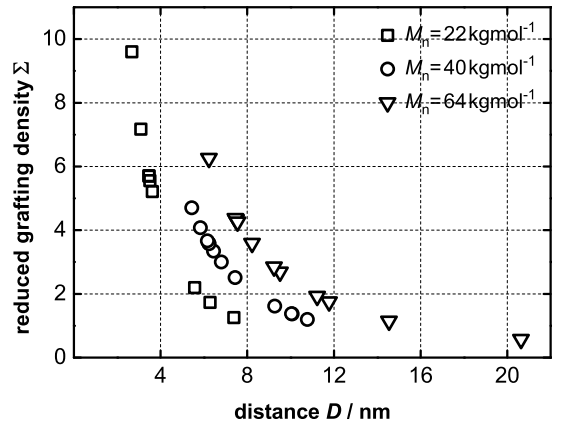

(a) Linear PS

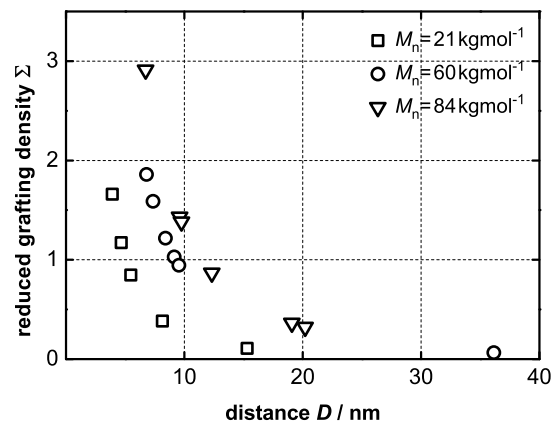

(b) Star shaped PS

Figure 4.5: Reduced grafting density $\Sigma$ and distance $D$ between the grafting points for (a) linear polystyrene and (b) four-arm star polystyrene with three different molar masses tethered onto gold substrates.

The right graph of Figure 4.5 shows the reduced grafting density and the corresponding distances of four-arm star polystyrene grafted onto gold substrates. Again, three different molar masses were investigated. The polymer films exhibited thicknesses up to $3 \mathrm{~nm}$. Regarding the increase of the degree of polymerization there is no clear tendency for the reduced grafting densities. For all three molar masses low and high values for $\Sigma$ were obtained. The maximum values for the reduced grafting densities for grafted linear polystyrene are between $\Sigma=6.3$ for the highest molar mass and $\Sigma=9.6$ for the lowest molar mass. The highest value for the reduced grafting density of star shaped polystyrene is at 2.9, which is significantly lower than for linear polymer chains. The star shaped polymers were synthesized via star RAFT agents using the R-group approach. 99] Through this approach, each arm of the star polymer contains a RAFT functionality at the terminal end that can be a possible anchoring group. The more RAFT groups are attached to the surface, the more space is occupied. Although 
a star shaped polymer with a comparable degree of polymerization has a smaller radius of gyration the four possible terminal anchoring groups can occupy more space on the substrate than a linear polymer chain with just one grafting point. Figure 4.6 shows linear grafted polymer chains and an exemplary grafting mode of a four-arm star shaped macromolecule.
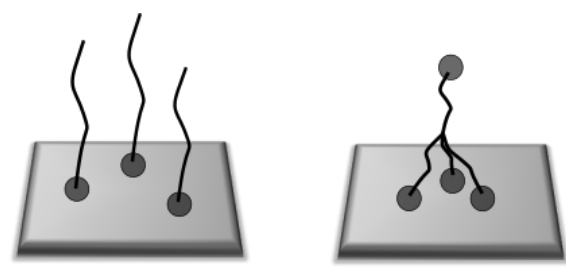

Figure 4.6: Linear polymer chains with one grafting point (left) compared to a four-arm star shaped macromolecule with multiple possible grafting points (right).

The comparison of the distances of both polymer topologies also yields information about hydrodynamic properties of linear and branched polymer architectures. Considering the same molar mass and the same reduced grafting density, distances between the grafting points are higher for linear polymer compared to the branched tethered macromolecule. This can be explained by the contraction of the branched architecture. Taking into account the distances between the grafting points and applying equation 4.4 leads to the following relation:

$$
\left(\frac{D_{\mathrm{br}}}{D_{\text {lin }}}\right)_{M, \Sigma}=g_{\mathrm{D}}=0.622 \pm 0.009 .
$$

The value of the contraction factor $g_{D}$ agrees well with published data of hydrodynamic studies on branched polystyrene in toluene. Douglas experimentally determined a contraction factor of 0.631 and compared it with theoretical simulation, which predicted a factor of 0.625. [111] The star shaped architecture leads to a smaller hydrodynamic volume due to topological constraints, so that the distances between grafted polymer molecules decrease as well. 


\subsubsection{Preliminary studies on constrained dewetting}

The following section gives an overview of different experimental conditions during the constrained dewetting procedure and the resulting impact on the morphology of the nanostructure. Parameters that were investigated are:

- Reversibility of the surface morphologies.

- Different solvent qualities.

- Fast versus slow dewetting.

- Solvent temperature during dewetting procedure.

- Thermal stability of the nanostructured surface.

\section{Reversibly tunable morphologies}

In this section the reversibility and modification of the morphology through different solvent qualities are explained.

The obtained structure on the substrate depends on the grafting density of the polymer and the quality of the solvent. These phenomena can be seen in Figure 4.7 which shows the dewetting process for a thin polystyrene layer of linear architecture with $\bar{M}_{\mathrm{n}}=60 \mathrm{~kg} \mathrm{~mol}^{-1}$ and $\Sigma=3.6$. If the polymer is immersed in a good solvent the polymer chains swell and stretch away from the surface. By drying the substrate, a unique polymer film should develop. The AFM image (a) in Figure 4.7 shows a homogeneous polystyrene layer after the immersion in toluene and finally drying. The roughness $R_{\mathrm{q}}$ of these structures was very low and ranged from $0.25 \mathrm{~nm}$ to $0.33 \mathrm{~nm}$. The difference in height that is shown in the color bar next to the AFM images varied around $2.5 \pm 0.2 \mathrm{~nm}$ for homogeneous morphologies.

When the same substrate was immersed again in toluene and the solvent quality was subsequently reduced by the addition of acetone, the structure shown in Figure 4.7 (b) was formed. The AFM image shows an irregular nanostructure consisting of separated micelles and worm-like formations. The roughness $R_{\mathrm{q}}$ and the difference in height were increasing to $0.66 \mathrm{~nm}$ and $4.7 \mathrm{~nm}$, respectively. The nanostructure could be reversibly converted to a homogeneous layer when the substrate was wetted with toluene again (see Figure $4.7(\mathrm{c})$ ). 
(a)

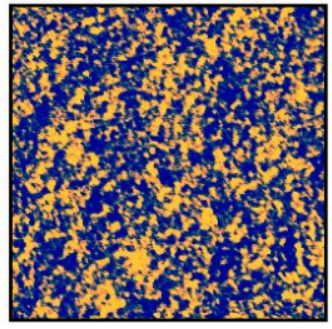

(b)

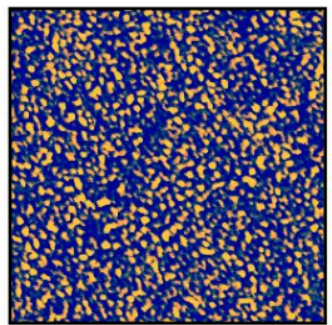

(c)

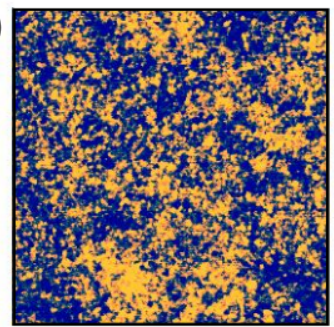

(d)

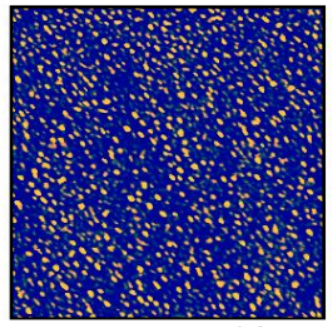

$1.0 \mu \mathrm{m}$

\section{$2.4 \mathrm{~nm}$}
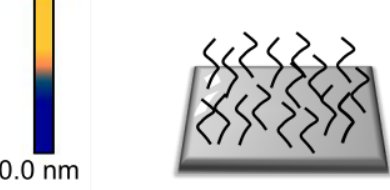

constrained

dewetting

with aceton

\section{$4.7 \mathrm{~nm}$}
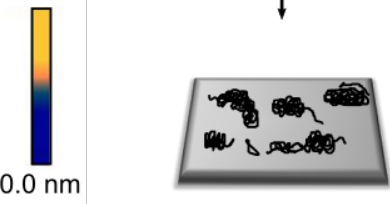

wetting

$2.5 \mathrm{~nm}$

with toluene
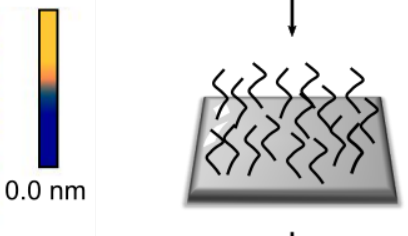

strained

dewetting

with water

\section{$6.8 \mathrm{~nm}$}
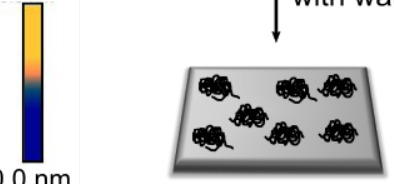

Figure 4.7: Constrained dewetting of linear polystyrene with two different solvents.

(a) homogeneous polymerlayer mediated by toluene. (b) nanostructure of polystyrene arranged with acetone (c) unique polymerlayer mediated by toluene after a dewetting process (d) nanostructure of polystyrene arranged with water. 
If the substrate was deposited in dimethylformamide and the solvent quality was subsequently reduced by the addition of water a regular morphology of micelles was obtained over extended areas. The corresponding AFM image is shown in Figure 4.7 (d). The constrained dewetting with dimethylformamide and water yielded the highest roughness $R_{\mathrm{q}}=0.94 \mathrm{~nm}$ and height difference of $6.8 \mathrm{~nm}$. Since water is a very poor solvent for polystyrene compared to acetone, the polymer chains are more constrained so that the formed features in water were quenched to a smaller feature diameter and a larger height. The nanostructures caused by constrained dewetting of polymer chains tethered to a surface are dependent on the chosen solvent conditions. ${ }^{[35,36]}$ In poor solvents the polymer chains collapse to avoid polymer-solvent interaction. This is balanced by the tendency of the polymer chains to stretch away from the surface. The poorer the solvent quality, the more prone are the polymer chains to minimize polymer-solvent interaction. The monomer-monomer interaction becomes effectively attractive during dewetting process. 36

The reversibility of the wetting and dewetting procedure and the corresponding structures was tested ten times and resulted in the same corresponding polymer morphology each time. This demonstrates the elegance of this method: To produce tunable nanostructured surfaces that can be initiated by just modifying the solvent composition. Furthermore the reversibility of the nanostructured surfaces over numerous times shows the strength of the gold-sulphur bond. The morphologies also depend on the grafting density and therefore it can be concluded that no polymer chains were cleaved from the surface through many dewetting operations.

\section{Dewetting, fast and slow}

According to literature the constrained dewetting procedure is described as the constant slow reduction of the solvent quality (slow dewetting). Therefore, the substrate with tethered polymer is immersed in a solvent that has high solvent-monomer interactions. The solvent quality is constantly reduced by adding solutions of both, good and bad solvent while the concentration of the poor solvent is further increased. Ultimately, the amount of the bad solvent dominates significantly. During preliminary studies of the constrained dewetting procedure a fast approach was investigated (fast dewetting). Here, the surface was immersed in a good solvent so the polymer chains could swell. The substrate was removed and a solvent layer on the substrate was retained. Finally, a minor amount of a poor 
solvent was dropped onto the sample. The obtained morphologies for linear polystyrene with $\bar{M}_{\mathrm{n}}=60 \mathrm{~kg} \mathrm{~mol}^{-1}$ and $\Sigma=1.8$ are shown for fast and slow dewetting in Figure 4.8. Constrained dewetting was conducted with dimethylformamide as the good solvent and water was used to reduce the solvent quality.

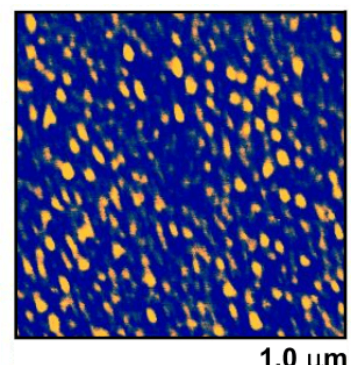

(a)

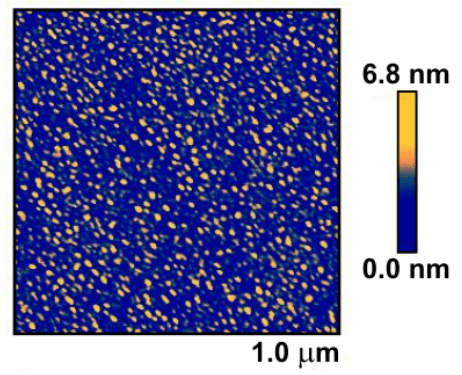

(b)

Figure 4.8: Nanostructured surfaces mediated by constrained dewetting of linear polystyrene $\left(\bar{M}_{\mathrm{n}}=60 \mathrm{~kg} \mathrm{~mol}^{-1}\right)$ with water. The left AFM image shows a slow dewetting process, whereas the right image shows a nanostructure formed via a fast dewetting method.

With both approaches a nanostructured surface could be obtained. Figure 4.8 (a) shows the nanopattern formed during the slow dewetting method. Big micelles with a considerable amount of irregularities were formed. The average feature size of these micelles was $d=55 \pm 10 \mathrm{~nm}$. The AFM image in Figure 4.8 (b) shows the obtained nanostructure after fast constrained dewetting. Smaller micelles formed with a feature size of $d=31 \pm 3 \mathrm{~nm}$. In addition the height difference of the structure is slightly larger for the smaller micelles than for the micelles formed during the slow dewetting process.

Immersion in a good solvent caused the polymer chains to stretch away from the surface and form a uniform layer. When the solvent quality was constantly reduced towards the poor solvent regime, the polymer chains collapsed to reduce solvent monomer interactions. If the solvent quality was quenched in a short time frame, the elastic stretched polymer chains were frozen in place thus high micelles with a small diameter were formed. However, if the solvent quality is reduced very slowly, the polymer chains still had the possibility to move and form the favoured structure with the lowest surface energy to decrease the solvent monomer interaction. A small 
number of big micelles occupy a smaller surface than a large number of micelles with a small diameter.

\section{Temperature dependence of solvent dewetting}

Furthermore, the influence of the solvent temperature during the dewetting procedure was studied. Since the solubility of polystyrene in water depends on the temperature, differences of the obtained polymer morphologies were investigated. Three solvent temperatures were measured, $\theta=0$, room temperature and $70{ }^{\circ} \mathrm{C}$. Figure 4.9 summarizes AFM images in 3D of grafted linear polystyrene with $\bar{M}_{\mathrm{n}}=22 \mathrm{~kg} \mathrm{~mol}^{-1}$ and a reduced grafting density of $\Sigma=1.2$ that was dewetted with nanopure water.

Figure 4.9 (a) shows the polymer morphology after dewetting with water at room temperature. The surface had a very high regularity and yielded the highest roughness $R_{\mathrm{q}}=1.02 \mathrm{~nm}$ and height difference of $6.6 \mathrm{~nm}$. On the other hand, the AFM images of structures formed with cold or hot water showed a less uniform structure. This is also evident comparing the roughness and the height difference collected in Table 4.1. Both $R_{\mathrm{q}}$ and $\Delta$ height for cold and hot water were lower than for the morphology resulting from dewetting at room temperature (rt). A lower temperature had an influence on the solubility of polystyrene in water. As previously mentioned, a poor solvent led to a morphology with a moderate regularity and high roughness. However, the temperature also has an influence on the mobility of the polymer chains. It is possible that a poorer flexibility of the chains led to irregular nanostructures because the formation of polymer domains to avoid solvent monomer interaction was hindered. The same argumentation can be made for the structure mediated by hot water. Here, the chains could be too flexible and thus a structure towards a homogeneous layer was formed. For comparison: A uniform polymer layer typically showed a roughness of approximately $R_{\mathrm{q}} \approx 0.3 \mathrm{~nm}$.

Table 4.1: Parameters of surface morphology to describe the influence of water temperature during dewetting process.

\begin{tabular}{cccc}
\hline$\theta /{ }^{\circ} \mathrm{C}$ & $R_{\mathrm{q}} / \mathrm{nm}$ & $\Delta$ height $/ \mathrm{nm}$ & regularity \\
\hline \hline 0 & 0.62 & 4.5 & low \\
$\mathrm{rt}$ & 1.02 & 6.6 & high \\
70 & 0.56 & 4.0 & low \\
\hline
\end{tabular}




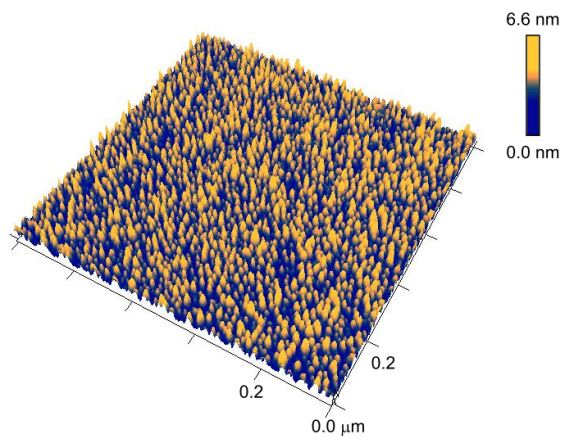

(a) room temperature

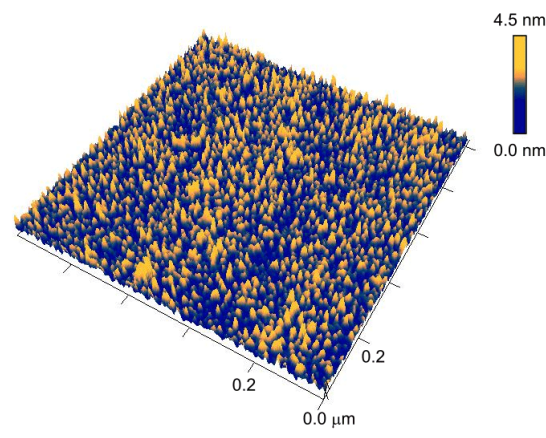

(b) $\theta=0{ }^{\circ} \mathrm{C}$

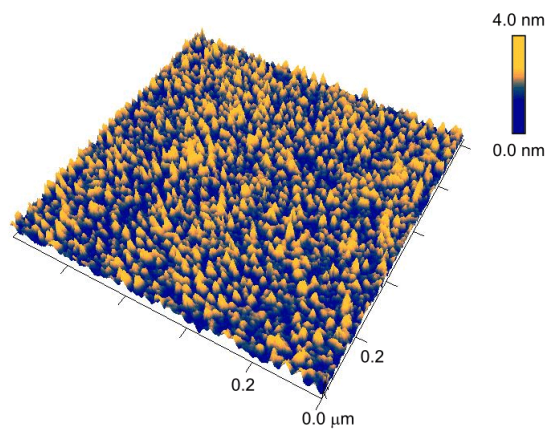

(c) $\theta=70^{\circ} \mathrm{C}$

Figure 4.9: AFM images of surface morphologies via constrained dewetting with dimethylformamide and different temperature water. (a) At room temperature. (b) Cold water at $\theta=0{ }^{\circ} \mathrm{C}$. (c) Hot water at $\theta=70^{\circ} \mathrm{C}$.

Furthermore, the temperature stability of a nanostructured surfaces was investigated. A polystyrene film with $\bar{M}_{\mathrm{n}}=22 \mathrm{~kg} \mathrm{~mol}^{-1}$ and $\Sigma=5.2$ was dewetted with room temperature water to form a wormlike nanopattern. Afterwards, the sample was tempered to $70{ }^{\circ} \mathrm{C}$ under argon for 48 hours. AFM measurements yielded the following results shown in Figure 4.10. After tempering the structure turned to a regular network of polymer domains. The height difference and $R_{\mathrm{q}}$ of the tempered surface decreased from $6.9 \mathrm{~nm}$ to $3.9 \mathrm{~nm}$ and $0.88 \mathrm{~nm}$ to $0.55 \mathrm{~nm}$, respectively. Because of the higher temperature the macromolecules were more flexible and could 
arrange in a favored uniform polymer layer. The height of the worm-like structure was decreasing because the polymer chains were migrating in the direction of the substrate. As a consequence, the domains grew in size until they formed a network. A further tempering would most likely lead to a homogeneous layer.

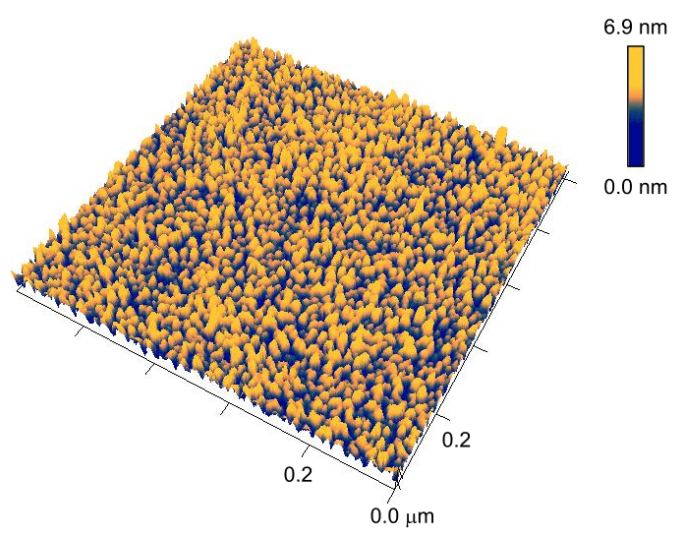

(a)

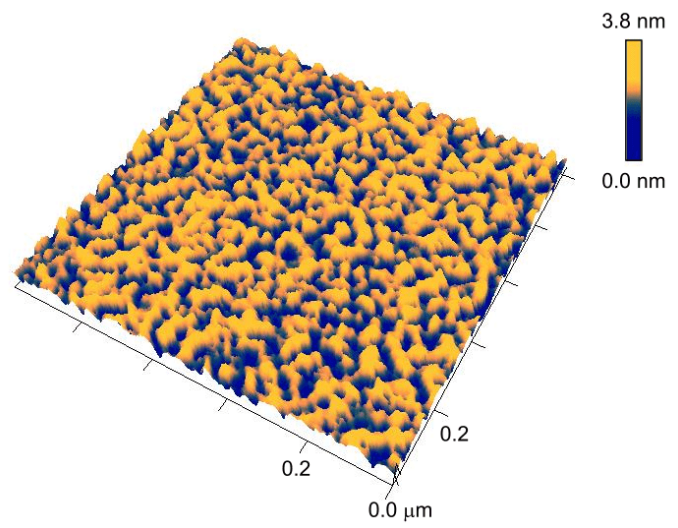

(b)

Figure 4.10: Representative AFM images to investigate the thermal stability of a nanostructured surface. (a) Wormlike structured polystyrene layer via constrained dewetting with water. (b) Formed structure after 48 hours tempering at $70{ }^{\circ} \mathrm{C}$. 


\subsubsection{Wettability of nanostructured surfaces}

It is known from literature that nanostructured surfaces can have an different wetting behaviour compared to the equivalent uniform layer. [113] It ranges between very high wettability with very low water contact angles and superhydrophobicity with contact angles around $150^{\circ}$. 114] These extremely hydrophobic surfaces are obtained when a nanostructured surface is thinly coated with a material of low surface energy. Using this procedure one can mimic surfaces that are known from nature, e.g. leaves with the so called lotus effect. [113] Surfaces that exhibit a high hydrophilicity are obtained by applying thin coatings of high-surface-energy materials to nanopatterned surfaces. [114]

Based on this knowledge, the influence of the structured polystyrene layer on the wetting behaviour was investigated. Therefore, samples of linear polystyrene with $\bar{M}_{\mathrm{n}}=40 \mathrm{~kg} \mathrm{~mol}^{-1}$ and different grafting densities on a gold surface were dewetted with water and analyzed using water contact angle measurements. Figure 4.11 gives a representative overview of three different structures and the corresponding water contact angles (WCA). Figure 4.11 (a) shows the AFM image of a polymeric structured surface where spherical micelles were predominant. The corresponding water contact angle was determined to $(78 \pm 2)^{\circ}$. The same sample but with a uniform polymer layer (see Figure 4.11 (c)) yielded a WCA of $(69 \pm 2)^{\circ}$ and represents a homogeneous polystyrene layer irrespective of the grafting density. Investigations of another sample that formed a network of polystyrene revealed a contact angle of $(81 \pm 2)^{\circ}$ and is shown in Figure 4.11 (b).

That means a nanostructured polystyrene layer increases the hydrophobicity compared to the uniform polystyrene substrate. Table 4.2 summarizes the obtained data and the calculated roughness $R_{\mathrm{q}}$. It is obvious that the water contact angle of the polystyrene surfaces were increasing related to $R_{\mathrm{q}}$. This phenomenon was first analyzed in 1936 by Wenzel, who observed different wettability properties for textile materials. ${ }^{[115,116]} \mathrm{He}$ also pointed out that the surface roughness is the primary parameter that has an influence on the hydrophilicity of a substrate. 
Table 4.2: Wettability properties of homogeneous and structured polystyrene surfaces.

\begin{tabular}{lccc}
\hline sample & $\Sigma$ & $R_{\mathrm{q}} / \mathrm{nm}$ & WCA \\
\hline \hline homogeneous & 1.0 & 0.29 & $69 \pm 2^{\circ}$ \\
\hline \multirow{2}{*}{ structured } & 1.0 & 1.31 & $78 \pm 2^{\circ}$ \\
& 3.6 & 1.53 & $81 \pm 2^{\circ}$ \\
\hline
\end{tabular}

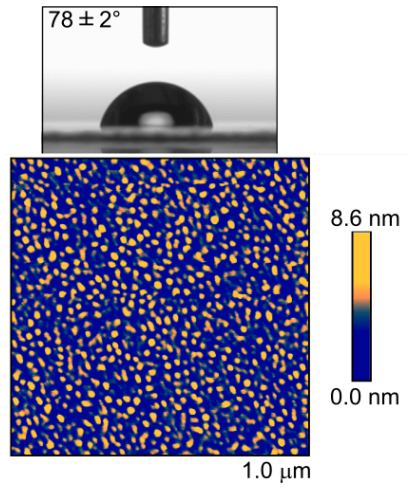

(a)

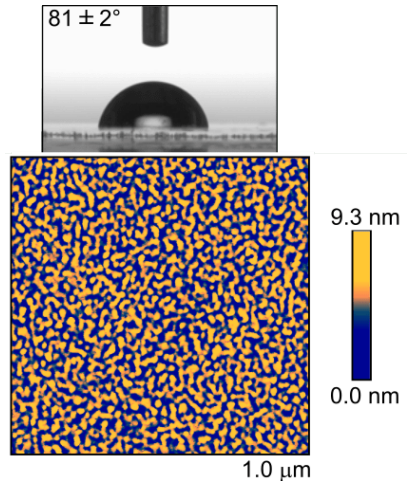

(b)

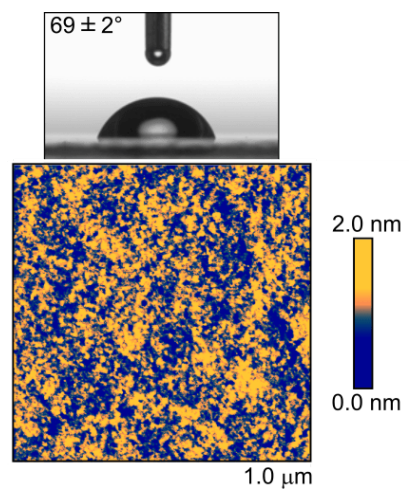

(c)

Figure 4.11: AFM images and wettability properties of homogeneous and structured polystyrene surfaces. (a) Nanostructure with spherical micelles, (b) a network of polymeric domains and (c) a uniform layer. 


\subsubsection{Conclusion}

In the last section it was pointed out that an ultraflat gold substrate is indispensable for the imaging of structured surfaces on a nanometer scale. The atomically flat metal surface was obtained by vapor deposition under ultrahigh vacuum and nominal evaporation rates. An anchoring of polystyrene with different architecture via the RAFT functionality was successful and could be analyzed by spectroscopic ellipsometry.

With these samples first constrained dewetting experiments could be conducted. The reversibility between homogeneous and structured surface via constrained dewetting with dimethylformamide and water was observed. Additionally, the specific morphology obtained by dewetting with water could be varied by modifying the solvent composition. Another parameter to successfully change the surface structure was the subsequent tempering of the substrate. This procedure led to a coalescence of the polymeric domains where a network appears to be a transition structure before a uniform polystyrene layer can form.

Furthermore, the constrained dewetting procedure was investigated with regards to solvent temperature during dewetting. An improvement was the development of a faster dewetting procedure that led to ordered nanostructures with a very high surface roughness.

Finally it was shown that changing the polymeric morphology could be used to modify the surface wettability.

In the following section the correlation between the reduced grafting density $\Sigma$ of polystyrene with different molar masses and topology and the formed nanostructure is discussed. Therefore, the improved fast constrained dewetting procedure with solvents at room temperature was used.

\subsection{Tunable nanostructures mediated by RAFT polymers}

The formation of nanostructures on gold surfaces was examined for two different polymer architectures. As starting point, linear polystyrene carrying a terminal RAFT group to mediate the binding to the gold substrate was chosen. After establishing this method to design nanostructured surfaces, four-arm star polystyrene was used and the influence of the different polymer architecture on the nanostructure formation was investigated. Polystyrene was synthesized via reversible addition-fragmentation chain transfer (RAFT) polymerization. The RAFT moiety at the terminal end 
of the polymer chain should also function as the anchoring group. Polymer with three different molar masses for each polymer architecture were used for film formation on gold substrates. The studied molar masses are summarized in table 4.3 .

Table 4.3: Studied molar masses of the linear and four-arm star polystyrene (PS).

\begin{tabular}{ccc}
\hline$/ \mathrm{kg} \mathrm{mol}^{-1}$ & linear PS & star PS \\
\hline \hline $\bar{M}_{\mathrm{n}, 1}$ & 21 & 22 \\
$\bar{M}_{\mathrm{n}, 2}$ & 40 & 60 \\
$\bar{M}_{\mathrm{n}, 3}$ & 64 & 84 \\
\hline
\end{tabular}

\section{Quantitative analysis of atomic force microscopy images}

The visual inspection of the nanostructure morphologies obtained from AFM measurements can be misleading. Therefore, a strategy to quantitatively analyze the differently structured domains needed to be developed. Using the image processing and analysis program ImageJ structures were specified through the calculation of circularities. Within this section, the image analyzing procedure is introduced. The AFM image corresponding to L6 in Figure 4.14 is used to demonstrate the analysis of the morphology.

Differently structured domains can be segmented by their circularity which is a parameter for the roundness of an object. The circularity of mathematical perfect circles is one, whereas the circularity of objects with edges and corners tend towards zero. Image $J$ calculates circularity $C$ using the following relationship:

$$
C=4 \pi \cdot \frac{\text { Area of domain }}{[\text { Perimeter of domain }]^{2}} .
$$

Using this ratio the formed nanostructure was segmented into three regimes that are presented in the following table 4.4 .

Further parameters that can be calculated by ImageJ are the number of the domains called counts, the averaged size in $\mathrm{nm}^{2}$ of the features and filled area in \%. Using the averaged size of spherical micelles the diameter can be determined by simple mathematical geometry of a circle $A=\pi(d / 2)^{2}$. The 
Table 4.4: Specification of differently structured domains. Circularities of obtained domains in thresholded AFM images were calculated using ImageJ. Calculations of their filled area led to the segmentation into three regimes.

\begin{tabular}{cl}
\hline Circularity & Morphology \\
\hline \hline $0.0-0.3$ & network \\
$0.3-0.7$ & worm-like micelles \\
$0.7-1.0$ & spherical micelles \\
\hline
\end{tabular}

calculated diameter $d$ represents at the same time the width of a worm-like domain or the width of a network, respectively. This procedure is possible because preliminary studies showed, that spherical micelles are not growing infinitely to bigger circles but connect with near micelles to a worm-like micelle which again fuse to a network structure. Figure 4.12 shows the corresponding masks of the analysis by circularity where (a) represents the original but thresholded AFM image. Figure (b) represents a segment that corresponds to a network with circularity ranging from $0.0-0.3$. In contrast to worm-like segments and spherical micelles shown in (c) and (d), the network structure covers the largest fraction of surface so that the nanostructure corresponding to L6 in Figure 4.14 was assigned to the network regime. The width of this feature was calculated to be $d=13 \mathrm{~nm}$ determined by the diameter of spherical micelles in Figure (d).

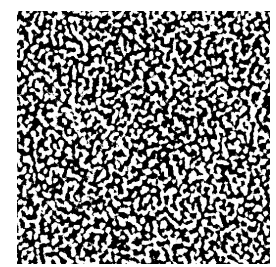

(a) $C=0-1.0$

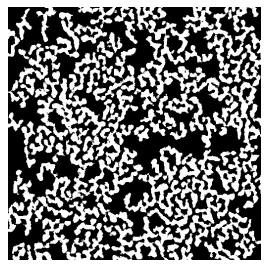

(b) $0.0-0.3$

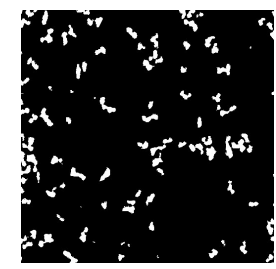

(c) $0.3-0.7$

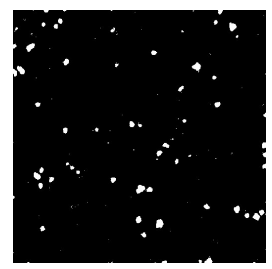

(d) $0.7-1.0$

Figure 4.12: Resulting masks of the segmentation concerning circularity which was calculated using ImageJ. (a) Original but thresholded AFM image. (b) Belongs to area that adopted network structures, (c) worm-like micelles and (d) corresponds to spherical micelles. 


\subsubsection{Linear polymer systems}

The morphology of a nanostructured surface formed by constrained dewetting depends on the grafting density of the tethered polymer. ${ }^{[35,36]}$ Thus, different grafting densities for each polymer were prepared and studied. The immobilization of the polymer was performed in toluene. The formation of nanostructured surfaces was achieved by constrained dewetting where dimethylformamide served as good solvent and nanopure water as poor solvent.

In the following sections the dependencies of the molar mass, grafting density, architecture of the macromolecules and the formed nanostructure is described. Three representative images of the obtained morphologies, which were obtained via atomic force microscopy, and the corresponding height histograms are shown. The depiction of the images was accomplished with the program NanoScope Analysis and the height histograms were plotted in Origin using the raw data. To give a quantitative evaluation, the Euler characteristic $\chi_{\mathrm{E}}$ was calculated using a python script. The image processing program ImageJ was applied to specify the formed structures. Here, the formed domains were investigated regarding their circularity and, in consequence, could be segmented in spherical micelles, worm-like micelles or network structures.

In the following, samples with anchored linear polystyrene with a molar mass of $\bar{M}_{\mathbf{n}}=21 \mathbf{~ k g ~ m o l}^{-1}$ on ultraflat gold substrates are described. The variation of the concentration of the polymer solution and reaction time led to different reduced grafting densities $\Sigma$ from 1.0 to almost 10 (see Figure 4.5). Constrained dewetting of those substrates with water as a poor solvent yielded differently structured surfaces on nanometer scale that could be analyzed with atomic force microscopy (AFM). Figure 4.13 shows representative AFM images of nanostructures of three substrates with increasing grafting densities from L1 to L3. Structures from substrates with $\Sigma$ lower than 1.0 could not be obtained. When $\Sigma$ reached a value of 1.3 a mixed structure of spherical micelles and worm-like features were obtained (see L1). These domains fused to a network of polystyrene with increasing grafting density (see L2 and L3). 
Table 4.5 summarizes the reduced grafting densities $\Sigma$ of the structured surfaces and the calculated Euler characteristic $\chi_{\mathrm{E}}$ - a number to describe a topological area's shape or structure - is indicated. For more theoretical details the reader is referred to Section 2.2.1.

Many separated micelles with a small area showed a large $\chi_{\mathrm{E}}$ whereas for network structures $\chi_{\mathrm{E}}$ tended towards zero or even negative values. The determination of $\chi_{\mathrm{E}}$ of nanostructures corresponding to L1 - L3 reflect this mathematical tendency. L1 counts the highest value of $\chi_{\mathrm{E}}=1560$ which corresponds to separated micelles whereas L2 and L3 form a polymeric network with an Euler characteristic of approximately 500. The parameters determined by a quantitative analysis using ImageJ are also presented in Table 4.5. Here, each AFM image was divided in different segments based on the circularity which represents a certain regime. For each regime that was contained in the image, the percentage area and averaged size in $\mathrm{nm}^{2}$ was calculated. Based on percentage area the dominating regime was determined. L1, the sample with the lowest reduced grafting density, corresponds to a structure dominated by worm-like micelles in contrast to the visual analysis of the AFM image where it appears to consist mostly of spherical micelles. The formed - and observed - polymeric networks in L2 and L3 were proved using the quantitative procedure. From the averaged size of spherical micelles that were extracted from each AFM image, the diameter of the domains was calculated. The nanostructures with low $\Sigma$ had a diameter of $8 \mathrm{~nm}$ whereas L3 showed a slightly larger diameter of $10 \mathrm{~nm}$.

All those structures have just a very short distance of $(9 \pm 5) \mathrm{nm}$ between the features and thus strictly separated domains could not be obtained for polystyrene with $\bar{M}_{\mathrm{n}}=21 \mathrm{~kg} \mathrm{~mol}^{-1}$. A polymer chain of this molar mass has a radius of gyration of approximately $5 \mathrm{~nm}$ under good solvent conditions. This small radius and the confinement in motion of the tethered chains on a flat surface led to the phenomenon that polymeric domains were not well separated and a large surface coverage was obtained. A short chain length is also responsible for the small height difference within the nanostructures as can be seen at the color bar next to the AFM images. No heights larger than $6.0 \mathrm{~nm}$ were achieved, which is also confirmed by the corresponding height histograms. These histograms hardly showed any differences for different nanostructures which is also an evidence for the confined polymer mobility. 
Table 4.5: Calculated reduced grafting density $\Sigma$, Euler characteristic $\chi_{\mathrm{E}}$ and domain size for morphologies resulting from constrained dewetting of RAFT functionalized linear polystyrene with $\bar{M}_{\mathbf{n}}=21 \mathbf{k g ~ m o l}^{-1}$.

\begin{tabular}{ccccccc}
\hline & $\Sigma$ & $\chi_{\mathrm{E}}$ & $\%$ area & $\begin{array}{c}\% \text { area } \\
\text { (dominat. regime) }\end{array}$ & diameter & distance \\
\hline \hline L1 & 1.3 & 1560 & $34 \%$ & $22 \%$ (worm-like) & $8 \mathrm{~nm}$ & $9 \pm 5 \mathrm{~nm}$ \\
L2 & 2.2 & 550 & $52 \%$ & $44 \%$ (network) & $8 \mathrm{~nm}$ & $9 \pm 5 \mathrm{~nm}$ \\
L3 & 5.2 & 500 & $60 \%$ & $53 \%$ (network) & $10 \mathrm{~nm}$ & $9 \pm 5 \mathrm{~nm}$ \\
\hline
\end{tabular}
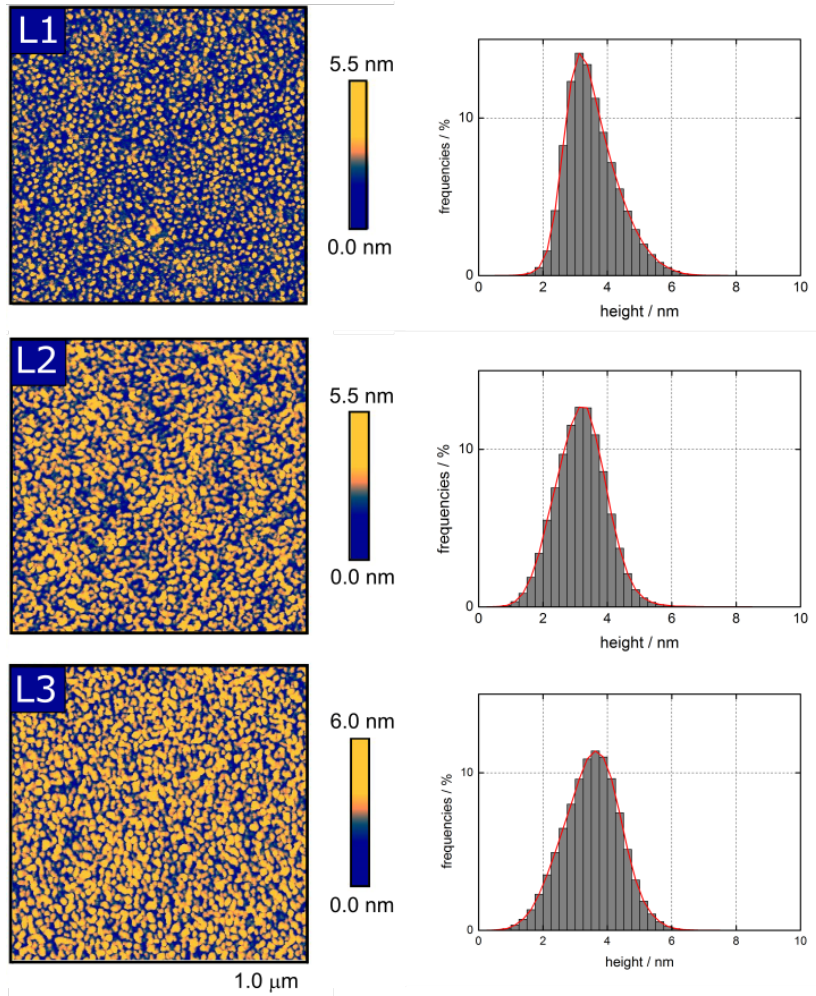

Figure 4.13: Nanostructured surfaces and corresponding height histograms mediated by constrained dewetting of RAFT functionalized linear polystyrene $\left(\bar{M}_{\mathrm{n}}=\mathbf{2 1} \mathbf{k g ~ m o l}^{-1}\right)$ with increasing grafting densities from L1 to L3. 
Then, samples with tethered linear polystyrene with $\bar{M}_{\mathrm{n}}=\mathbf{4 0} \mathrm{kg} \mathrm{mol}^{-1}$ were investigated. Figure 4.14 shows the dependence of grafting density and the formed nanostructures after constrained dewetting with water. With increasing grafting density from L4 to L6 the surface coverage was growing and all three structural regimes, from spherical to worm-like micelles and finally network, were obtained.

Table 4.6 summarizes calculated parameters from the quantitative analysis using the mathematical description by Minkowski measures and the image processing program ImageJ. With increasing reduced grafting density $\Sigma$ the surface topography changed which is demonstrated by the percentage area of the dominating regime. Compared to the samples with a molar mass of $\bar{M}_{\mathrm{n}}=21 \mathrm{~kg} \mathrm{~mol}^{-1}$ where $\Sigma=1.3$ led to worm-like micelles with a diameter of $8 \mathrm{~nm}$, the lowest $\Sigma$, which is $\Sigma=1.6$ for $\bar{M}_{\mathrm{n}}=40 \mathrm{~kg} \mathrm{~mol}^{-1}$ predominantly resulted in spherical micelles with $d=18 \mathrm{~nm}$. The same tendency is observed for the other reduced grafting densities. The diameters of the domains and also the distances between the domains are much larger than for the shorter chain length. It is therefore concluded that well separated polymeric features were obtained. Because of the larger chain length the polymer chain is less constrained by the anchoring to a surface. This led to higher distances and even to larger heights within the structure. Also, the corresponding height histograms show a different behaviour for each morphology which proves more freedom of movement for the longer polymer chains. The shoulders within the distributions indicate the transition between the regimes.

The height differences within the AFM images indicated by the color bar show different maximum values and no clear tendency at first appearance. One possible explanation is demonstrated in Figure 4.15. Here, the three morphologies and a possible progression of the maximum height difference is shown. The height of the polymeric features correlates with the volume of the structure. With increasing grafting density $\Sigma$ the volume and thus the height of the spherical micelle becomes larger. When a certain maximum height is reached, it is thermodynamically favorable for the structure to expand along the surface instead of arising in height. At this point, spherical micelles can fuse to worm-like micelles. Then, the worm-like micelles are increasing in size until they turn to a network with increasing volume. It should be pointed out that the figure only represents a model to explain the correlation and all heights and gradients are arbitrary. The same tendency but for another molar mass is shown in Appendix A. in Figure A1. 
Table 4.6: Calculated reduced grafting density $\Sigma$, Euler characteristic $\chi_{\mathrm{E}}$ and parameter determined using ImageJ for morphologies resulting from constrained dewetting of RAFT functionalized linear polystyrene with $\bar{M}_{\mathrm{n}}=40 \mathrm{~kg} \mathrm{~mol}^{-1}$.

\begin{tabular}{ccccccc}
\hline & $\Sigma$ & $\chi_{\mathrm{E}}$ & $\%$ area & $\begin{array}{c}\% \text { area } \\
\text { (dominat. regime) }\end{array}$ & diameter & distance \\
\hline \hline L4 & 1.6 & 850 & $24 \%$ & $15 \%$ (spherical) & $18 \mathrm{~nm}$ & $13 \pm 8 \mathrm{~nm}$ \\
L5 & 2.5 & 570 & $36 \%$ & $21 \%$ (worm-like) & $15 \mathrm{~nm}$ & $21 \pm 11 \mathrm{~nm}$ \\
L6 & 3.6 & 140 & $49 \%$ & $40 \%$ (network) & $13 \mathrm{~nm}$ & $19 \pm 6 \mathrm{~nm}$ \\
\hline
\end{tabular}
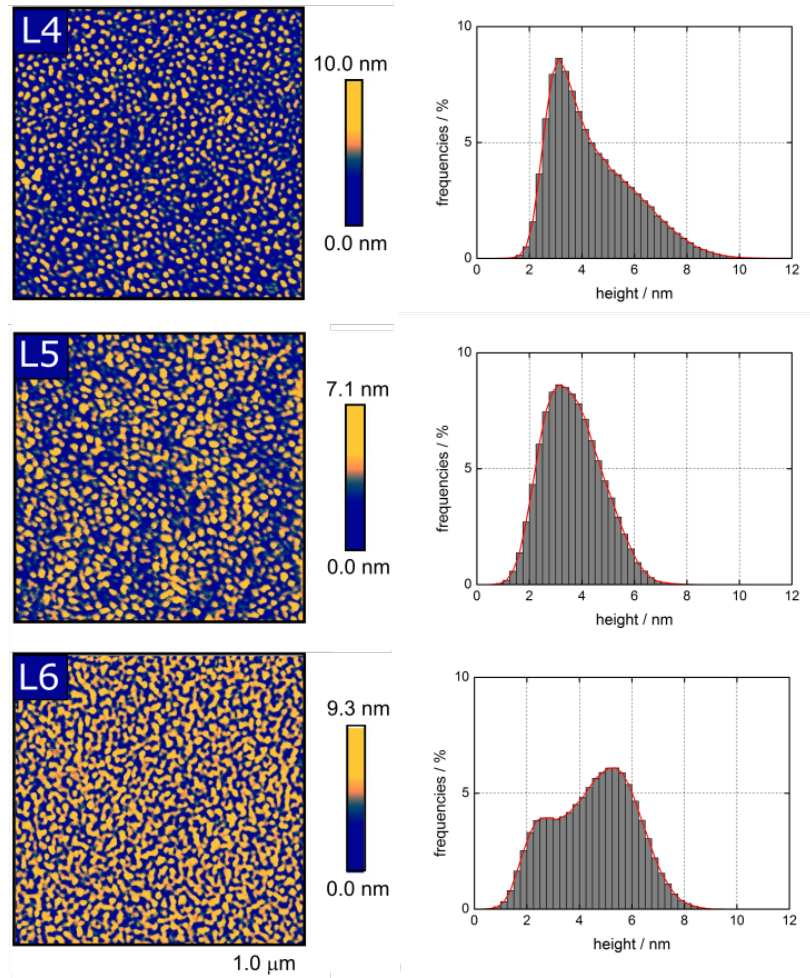

Figure 4.14: Nanostructured surfaces and corresponding height histograms mediated by constrained dewetting of RAFT functionalized linear polystyrene $\left(\bar{M}_{\mathrm{n}}=\mathbf{4 0} \mathrm{kg} \mathrm{mol}^{-1}\right)$ with increasing grafting densities from L4 to L6. 


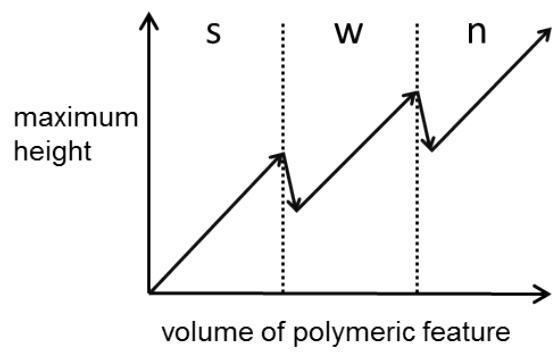

Figure 4.15: Gedanken experiment to explain teh dependence between the maximum height obtained from AFM images the formation of spherical micelles (s), worm-like micelles (w) and network structure (n). With increasing reduced grafting density the volume and thus the height of the domains become larger. When a certain maximum height is reached, it is thermodynamically favorable for the structure to expand along the surface instead of arising in height. At this point, the domains transition into the another regime.

The third investigated linear polystyrene exhibited a molar mass of $\bar{M}_{\mathrm{n}}=64 \mathrm{~kg} \mathrm{~mol}^{-1}$. Three representative AFM images are shown in Figure 4.16, further images with different reduced grafting densities are summarized in Appendix A in Figure A1.

The AFM images are again organized with increasing grafting density from L7 to L9 and show a transition from spherical micelles to worm-like domains. The transition could also be observed in the height histograms. The height distribution of sample L7 shows a sharp peak whereas the other samples L8 and L9 each show two maxima.

Table 4.7 shows calculated parameters from the quantitative analysis using the mathematical description by Minkowski measures and the image processing program ImageJ. The lowest reduced grafting density of $\Sigma=0.6$ shows the highest value of Euler characteristic with $\chi_{\mathrm{E}}=3020$ and predominantly led to spherical micelles. $90 \%$ of the percentage surface area was identified to approximately perfect circles with a circularity of 0.9 and a diameter of $9.4 \mathrm{~nm}$. The threefold grafting density of 1.9 (sample L8) led to spherical micelles as well and the diameter increased to $17.8 \mathrm{~nm}$. The average size of the micelles increased from $70 \mathrm{~nm}^{2}$ to $250 \mathrm{~nm}^{2}$ which coincides with the threefold enlargement of $\Sigma .10 \%$ of the surface coverage of sample L8 correspond to worm-like micelles which can be assigned to the shoulder in the height histogram. 
Table 4.7: Calculated reduced grafting density $\Sigma$, Euler characteristic $\chi_{\mathrm{E}}$ and domain size for morphologies resulting from constrained dewetting of RAFT functionalized linear polystyrene with $\bar{M}_{\mathrm{n}}=64 \mathrm{~kg} \mathrm{~mol}^{-1}$.

\begin{tabular}{ccccccc}
\hline & $\Sigma$ & $\chi_{\mathrm{E}}$ & $\%$ area & $\begin{array}{c}\% \text { area } \\
\text { (dominat. regime) }\end{array}$ & diameter & distance \\
\hline \hline L7 & 0.6 & 3020 & $9 \%$ & $8 \%$ (spherical) & $9 \mathrm{~nm}$ & $39 \pm 16 \mathrm{~nm}$ \\
L8 & 1.9 & 940 & $29 \%$ & $18 \%$ (spherical) & $18 \mathrm{~nm}$ & $17 \pm 8 \mathrm{~nm}$ \\
L9 & 4.4 & 350 & $34 \%$ & $17 \%$ (worm-like) & $18 \mathrm{~nm}$ & $18 \pm 9 \mathrm{~nm}$ \\
\hline
\end{tabular}
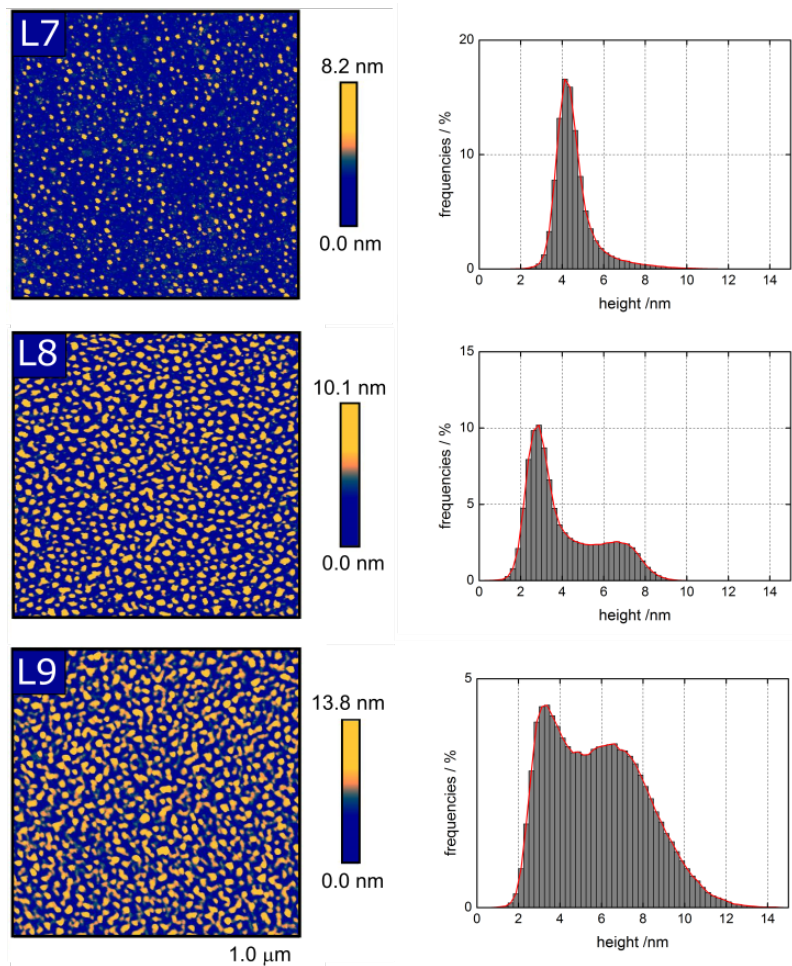

Figure 4.16: Nanostructured surfaces mediated by constrained dewetting of RAFT functionalized linear polystyrene $\left(\bar{M}_{\mathrm{n}}=64 \mathbf{k g ~ m o l}^{-1}\right)$ with increasing grafting densities from L7 to L9. 
The topological analysis of L9, the sample with the highest reduced grafting density of $\Sigma=4.4$, was determined to be a mixture of all three regimes. The classification concerning the circularity of the domains is shown in Figure 4.17. Domains with edges and corners like networks attain to circularities towards zero whereas perfect circles tend towards one. The surface coverage is divided in $17 \%$ worm-like micelles, $9 \%$ spherical micelles and $7 \%$ a polymeric network. This leads to the significant broadening of the height distribution.

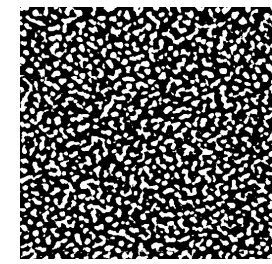

(a) $C=0-1.0$

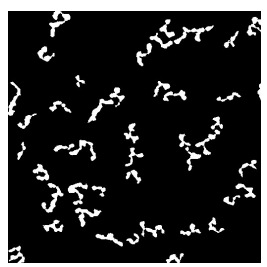

(b) $0.0-0.3$

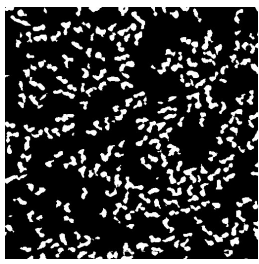

(c) $0.3-0.7$

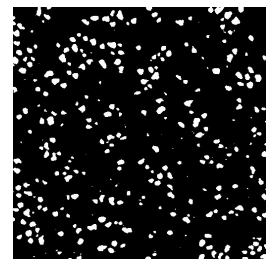

(d) $0.7-1.0$

Figure 4.17: Resulting masks of the segmentation by circularity using ImageJ. (a) Original but thresholded AFM image of sample L9. (b) - (d) Masks corresponding to different circularities $C$, that allows for the specification of the obtained nanostructure. Domains are divided in (b) $7 \%$ a polymeric network, (c) $17 \%$ worm-like micelles and (d) $9 \%$ spherical micelles.

\section{Tuning the morphology by modifying the solvent composition}

In the previous section structured surfaces via constrained dewetting with dimethylformamide as good solvent and water as the poor solvent were introduced. The morphology of the nanostructure is dependent on the grafting density of the anchored polymer chains and the behaviour of the polymeric species in solution. ${ }^{[35,36}$ Under good solvent conditions polymer chains swell to a uniform layer. By decreasing the solvent quality swollen polymer chains collapse to reduce the interaction between polymeric species and solvent. Therefore, the solvent quality during constrained dewetting affects the type of nanopattern. ${ }^{35]}$

Three different molar masses of RAFT functionalized linear polystyrene were investigated concerning the modification of the morphology by changing the solvent composition. First, the formed nanostructures of samples with different reduced grafting densities of linear polystyrene with $\bar{M}_{\mathrm{n}}=21,40$ and $64 \mathrm{~kg} \mathrm{~mol}^{-1}$ dewetted with water were determined. Sec- 
ond, the same samples were reversibly turned into a uniform layer by the immersion in toluene and finally dewetted with aceton. The resulting structures were recorded by atomic force microscopy.

For low molar masses and reduced grafting densities, most nanopatterns turned into a uniform layer with a low roughness. However, for large reduced grafting densities the nanopattern could be modified to a holey polymer layer. A representative AFM image is shown in Figure 4.18. Here, a network of linear polystyrene with $\bar{M}_{\mathrm{n}}=21 \mathrm{~kg} \mathrm{~mol}^{-1}$ after dewetting with water turned into a dense network with a higher surface coverage after dewetting with aceton. Those dense networks are also often called holey polymer layer. ${ }^{[35]}$ Because of the higher surface coverage the height difference within the nanostructure decreased from $6.0 \mathrm{~nm}$ to $4.3 \mathrm{~nm}$ since the height is proportional to the volume of the polymer layer.
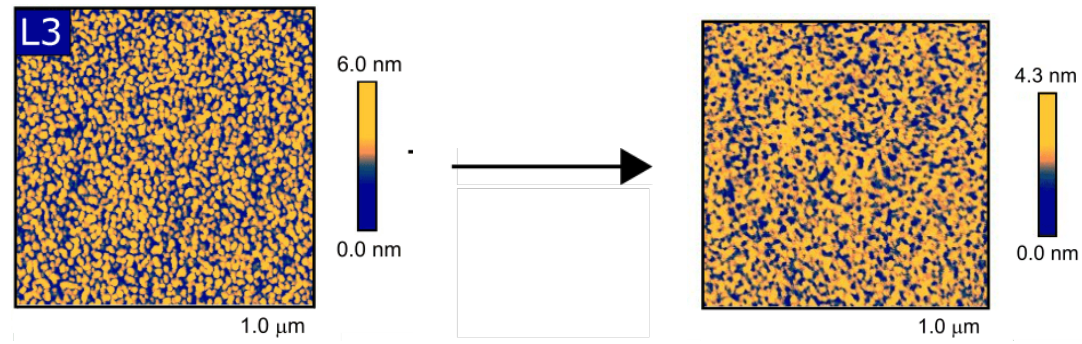

Figure 4.18: Arrangement of grafted polystyrene $\left(\bar{M}_{n}=21 \mathrm{~kg} \mathrm{~mol}^{-1}\right)$. Left AFM image shows the morphology after constrained dewetting with water and right image represents its modification after dewetting with aceton.

The transition was also quantified by the Euler characteristic that changed from $\chi_{\mathrm{E}}=500$ for the water dewetted surface to $\chi_{\mathrm{E}}=-167$ for the holey layer. The Euler characteristic tends to negative values, the more pixel are occupied by polymer. However, the perimeter of the nanostructure has to decrease while the filled area is increasing. This observation was proved by the quantitative analysis with ImageJ. Calculated parameters are summarized in Table 4.8. Both methods yielded a high surface coverage by the tethered polymer. In case of the water dewetted surface the nanopattern consisted of a network at a high percentage and partially featured spherical and worm-like micelles. In case of the aceton dewetted surface, however, $100 \%$ of the patterned polymer layer consisted of the network structure. The perimeter at the same time decreased from $88100 \mathrm{~nm}$ for the mixed structure 
to $11000 \mathrm{~nm}$ for the dense network. Figure 4.19 shows the percentage area occupied by the network structure. The fragmentation in spherical, wormlike micelles and network was achieved by the circularity as done in the previous sections. All micelles were then deducted and only the parts of the nanopattern consisting of network structures became visible. In Figure 4.19 (a) the water dewetted surface is shown. The subtraction of micelles led to larger black spots whereas Figure 4.19 (b) matches the corresponding AFM image in Figure 4.18 .

Table 4.8: Calculated Euler characteristic $\chi_{\mathrm{E}}$, the percentage area of the polymer, the percentage area of the network structured layer and the perimeter of morphologies mediated by different solvent compositions. The determined parameters belong to samples with RAFT functionalized linear polystyrene with $\bar{M}_{\mathrm{n}}=21 \mathrm{~kg} \mathrm{~mol}^{-1}$ and $\Sigma=4.4$.

\begin{tabular}{ccccc}
\hline dewetting solvent & $\chi_{\mathrm{E}}$ & $\%$ total area & $\%$ area network & perimeter \\
\hline \hline water & 500 & $60 \%$ & $53 \%$ & $88100 \mathrm{~nm}$ \\
aceton & -167 & $66 \%$ & $66 \%$ & $11000 \mathrm{~nm}$ \\
\hline
\end{tabular}

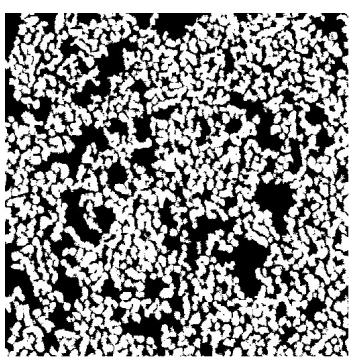

(a)

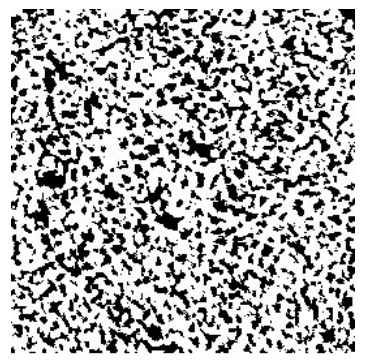

(b)

Figure 4.19: Network fragments corresponding to sample L3. (a) Percentage area of network structure after dewetting with water. (b) Percentage area of network structure after dewetting with aceton.

The broadening of the nanostructure was obtained for high reduced grafting densities for $\bar{M}_{\mathrm{n}}=21$ and $40 \mathrm{~kg} \mathrm{~mol}^{-1}$. For linear polystyrene with a higher molar mass of $\bar{M}_{\mathrm{n}}=64 \mathrm{~kg} \mathrm{~mol}^{-1}$, the broadening of the morphology for almost any grafting density could be obtained. Figure 4.20 
shows a representative AFM image of the modified morphology initiated by a different solvent composition. The AFM image at the left side shows the nanopattern after water dewetting. $62 \%$ of the covered surface were spherical micelles with a diameter of $18 \mathrm{~nm}$. Constrained dewetting with aceton resulted in a nanopattern that predominantly consisted of worm-like micelles and a network with $50 \%$ and $30 \%$ area of the covered surface, respectively. The diameter of the domains increased to $21 \mathrm{~nm}$ whereas the height decreased from $10.1 \mathrm{~nm}$ to $8.3 \mathrm{~nm}$.
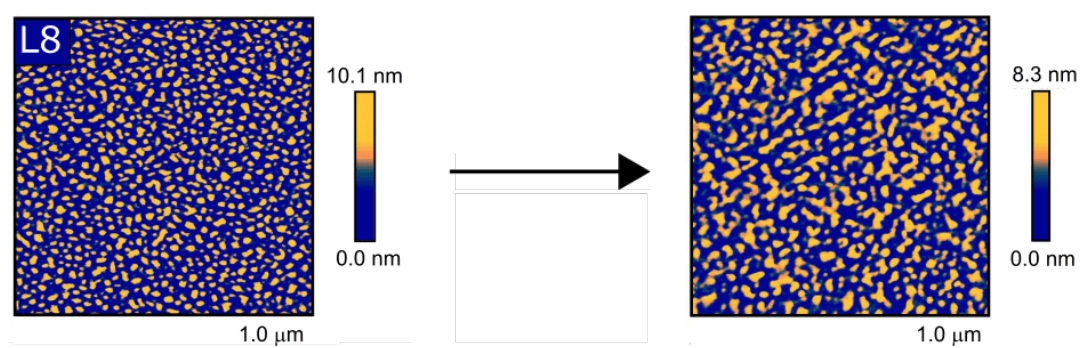

Figure 4.20: Arrangement of grafted polystyrene $\left(\bar{M}_{\mathrm{n}}=64 \mathrm{~kg} \mathrm{~mol}{ }^{-1}\right)$. Left AFM image shows the morphology after constrained dewetting with water and right image represents its modification after dewetting with aceton.

\subsubsection{Star polymer systems}

The morphology of a nanostructured surface formed by constrained dewetting mainly depends on the reduced grafting density of the tethered polymer. ${ }^{[35] 36]}$ As an additional parameter, different polymer topologies were investigated. The last section presented the investigations on linear polystyrene with three different molar masses. In this section formed nanopatterns mediated by four-arm star RAFT polystyrene will be introduced. The star shaped polymer was obtained via R-star RAFT agent and hence exhibits one RAFT moiety at the terminal end of each arm. Consequently four groups to anchor onto a surface are available. Star shaped polymers with three different molar masses were investigated. Ideally, all arms of these polymers exhibit similar chain lengths due to the controlled nature of the RAFT mechanism. The polymers were anchored onto ultraflat gold surfaces with different grafting densities. The immobilization of the polymer was performed in toluene. The formation of nanostructured sur- 
faces was achieved by constrained dewetting where dimethylformamide served as good solvent and nanopure water as poor solvent.

In the following sections the correlation of the molar mass, grafting density, architecture of the macromolecule and the formed nanostructure is described. Three representative images of the obtained morphologies that were obtained by atomic force microscopy and the corresponding height histograms are shown. The depiction of the images was accomplished with the program NanoScope Analysis whereas the height histograms are plotted in Origin using the raw data. To give a quantitative assertion the Euler characteristic $\chi_{\mathrm{E}}$ was calculated using a python script. The image processing program ImageJ was applied for further analysis of the nanostructures as described in the previous sections.

The immobilization of four-arm star RAFT polystyrene with a molar mass of $\bar{M}_{\mathbf{n}}=\mathbf{2 2} \mathbf{~ k g ~ m o l}^{-1}$ led to low reduced grafting densities $\Sigma$ ranging from 0.1 to 1.7. With a contraction factor of approximately 0.6 , the radius of gyration decreased from $4.7 \mathrm{~nm}$ for linear polystyrene to $2.8 \mathrm{~nm}$ for star shaped polymer chains. ${ }^{[111]}$ However, even at low grafting densities constrained dewetting with water led to structures with a high surface coverage. AFM images of the obtained morphologies for different grafting densities are collected in Figure 4.21 where $\Sigma$ is increasing from sample S1 to S3. The corresponding Table 4.9 summarizes calculated Euler characteristics $\chi_{\mathrm{E}}$, percentage total area and percentage area of the dominating regime.

Low and high grafting densities showed a high surface coverage of nearly $50 \%$ or even higher. Every formed structure exhibited a network as the major component. This fact is attributed to the low molar mass and the larger confinement due to the star topology. The possible anchoring on the flat surface over each arm led to a stronger immobilization but was opposed by the mobility penalty. Thus, networks consisting of domains with small averaged areas and very low height differences were formed.

The Euler characteristic $\chi_{\mathrm{E}}$ decreased with increasing $\Sigma$ as was expected. The samples S3 and L1 with the same molar mass and reduced grafting density but different architecture led to completely different structures. Grafted macromolecules with star topology exclusively formed a dense network after constrained dewetting with water. Linear polystyrene, however, formed $61 \%$ worm-like and $21 \%$ spherical micelles of the total covered area. The ratio of (perimeter)/(number of connected components) gives the perimeter per domain. Sample S3 was calculated to have $115 \mathrm{~nm}$ per 
Table 4.9: Calculated reduced grafting density $\Sigma$, Euler characteristic $\chi_{\mathrm{E}}$ and parameter determined using ImageJ for morphologies resulting from constrained dewetting of four-arm star polystyrene with $\bar{M}_{\mathrm{n}}=22 \mathrm{~kg} \mathrm{~mol}^{-1}$. RAFT functionalized linear polystyrene L1 with the same molar mass is shown for comparison.

\begin{tabular}{ccccc}
\hline & $\Sigma$ & $\chi_{\mathrm{E}}$ & $\%$ area & $\%$ area (dominat. regime) \\
\hline \hline S1 & 0.4 & 1010 & $45 \%$ & $37 \%$ (network) \\
S2 & 0.8 & 390 & $46 \%$ & $38 \%$ (network) \\
S3 & 1.2 & -180 & $61 \%$ & $61 \%$ (network) \\
\hline L1 & 1.3 & 1560 & $34 \%$ & $17 \%$ (worm-like) \\
\hline
\end{tabular}
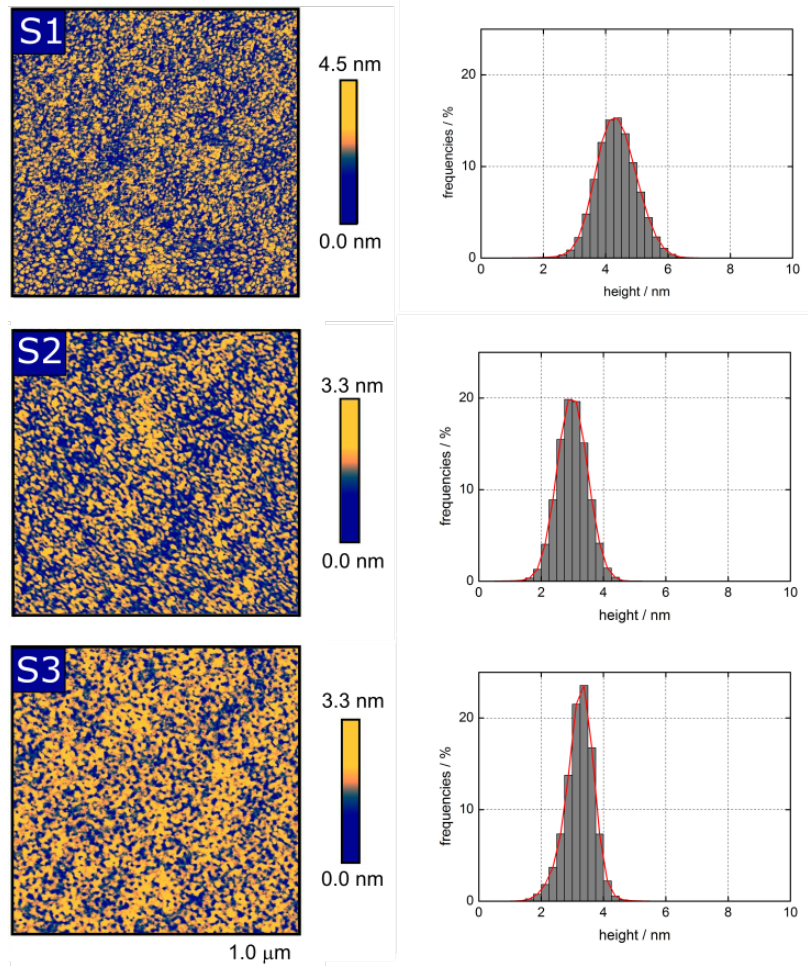

Figure 4.21: Nanostructured surfaces mediated by constrained dewetting of four-arm star polystyrene $\left(\bar{M}_{\mathrm{n}}=\mathbf{2 2} \mathbf{k g ~ m o l}^{-1}\right)$ with increasing grafting densities from S1 to S3. 
domain while L1 had $50 \mathrm{~nm}$ per domain. The fact that $\Sigma$ had the same value but the structure consisted of domains with a higher perimeter reflects, that the branched polymer molecule with more than one anchoring point is more constrained. This is illustrated in Figure 4.22.

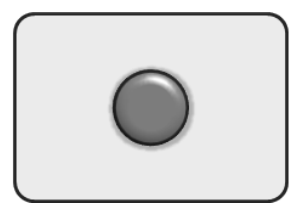

(a) Perimeter of linear polymer

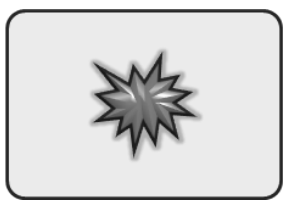

(b) Perimeter of star polymer

Figure 4.22: Schematic depiction of resulting perimeters per domain for polymer molecules with different architecture. Due to multiple possible anchoring groups at the star polymer, a domain with more edges and corners is formed.

More nanostructures mediated by constrained dewetting of grafted fourarm star polystyrene with $\bar{M}_{\mathrm{n}}=22 \mathrm{~kg} \mathrm{~mol}^{-1}$ were investigated by AFM and ImageJ. The corresponding images and calculated parameters are collected in Appendix A in Figure A2. It should be noted, that for samples with $\bar{M}_{\mathrm{n}}=22 \mathrm{~kg} \mathrm{~mol}^{-1}$, the calculation of the diameter and distance between the polymeric features was not possible.

The second investigated molar mass of star shaped polystyrene was $\bar{M}_{\mathrm{n}}=\mathbf{6 0} \mathrm{kg} \mathrm{mol}^{-\mathbf{1}}$. For substrates immobilized with these polymer molecules, constrained dewetting with water led to ordered nanostructures depending on the reduced grafting densities. In Figure 4.23 representative AFM images of structured surfaces and corresponding height histograms are shown. A very low reduced grafting density of $\Sigma=0.1$ predominantly resulted in spherical micelles with $\chi_{\mathrm{E}}=1240$. Nanostructures with a very high grafting density of $\Sigma=1.9$ formed a network at a very high percentage. Substrates with intermediate $\Sigma$ yielded mixed structures of all possible domains. Sample S5 with $\Sigma=1.2$ led to a surface coverage of worm-like micelles with $45 \%$. The residual nanostructure consisted of spherical micelles and networks. The calculated diameter of the domains increased while the distance between the features consequently decreased. 
Table 4.10: Calculated reduced grafting density $\Sigma$, Euler characteristic $\chi_{\mathrm{E}}$ and domain size for morphologies resulting from constrained dewetting of four-arm star polystyrene with $\bar{M}_{\mathrm{n}}=60 \mathrm{~kg} \mathrm{~mol}^{-1}$.

\begin{tabular}{ccccccc}
\hline & $\Sigma$ & $\chi_{\mathrm{E}}$ & $\%$ area & $\begin{array}{c}\% \text { area } \\
\text { (dominat. regime) }\end{array}$ & diameter & distance \\
\hline \hline S4 & 0.1 & 1240 & $20 \%$ & $16 \%$ (spherical) & $14 \mathrm{~nm}$ & $21 \pm 11 \mathrm{~nm}$ \\
S5 & 1.2 & 520 & $33 \%$ & $15 \%$ (worm-like) & $17 \mathrm{~nm}$ & $16 \pm 6 \mathrm{~nm}$ \\
S6 & 1.9 & 30 & $69 \%$ & $69 \%$ (network) & $18 \mathrm{~nm}$ & $9 \pm 3 \mathrm{~nm}$ \\
\hline
\end{tabular}
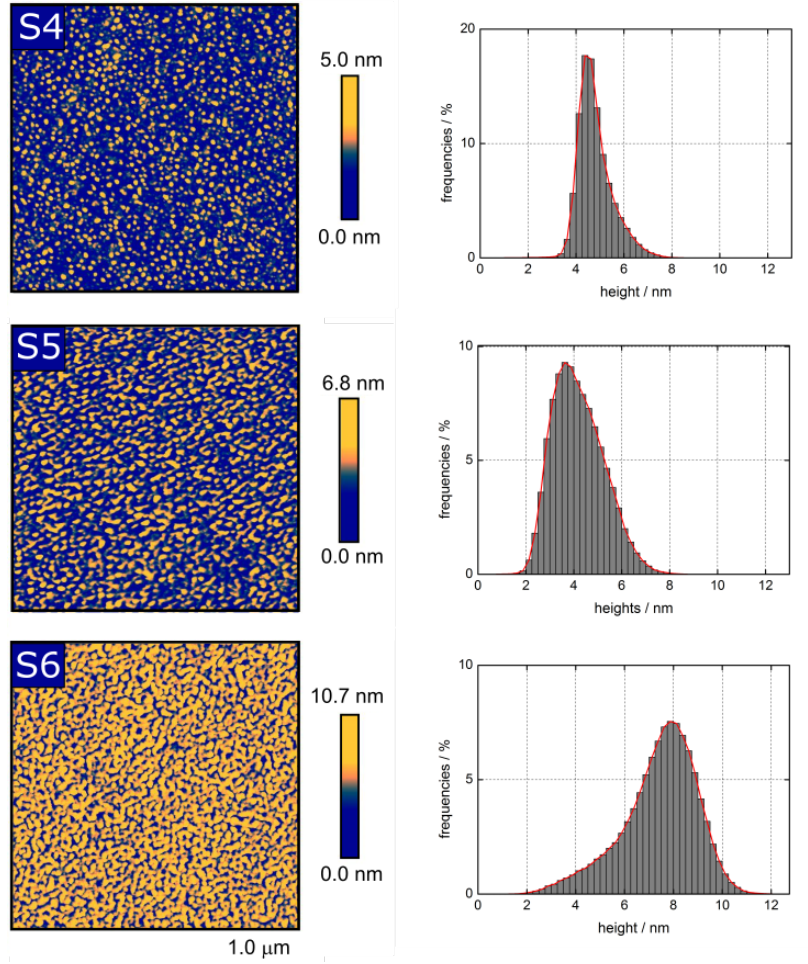

Figure 4.23: Nanostructured surfaces mediated by constrained dewetting of four-arm star polystyrene $\left(\bar{M}_{\mathrm{n}}=\mathbf{6 0} \mathrm{kg} \mathrm{mol}^{-\mathbf{1}}\right)$ with increasing grafting densities from S4 to S6. 
In the following, two samples of the same molar mass and same reduced grafting density $\Sigma$ but different polymer architecture, linear and fourarm star polystyrene, will be compared. Both samples were dewetted with water in the same manner. As a reminder, to compare different polymer architectures, the reduced grafting density $\Sigma$ was introduced. $\Sigma$ is proportional to the root mean square radius of gyration $\left\langle s^{2}\right\rangle^{1 / 2}$ that describes the dimensions of a polymer chain under specific temperature and solvent conditions. Thus, $\Sigma$ is defined as the number of macromolecules that occupy a defined area on the surface compared to the area that a free, non-interacting polymer molecule occupies under the same experimental conditions. In Figure 4.24 the composition of the obtained nanostructures for linear and star shaped polystyrene with $\Sigma=1$ and $\bar{M}_{\mathrm{n}}=60 \mathrm{~kg} \mathrm{~mol}^{-1}$ are shown.
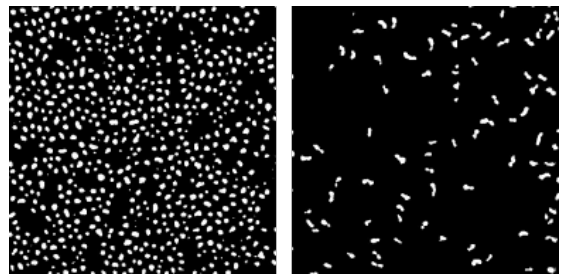

(a) Linear PS

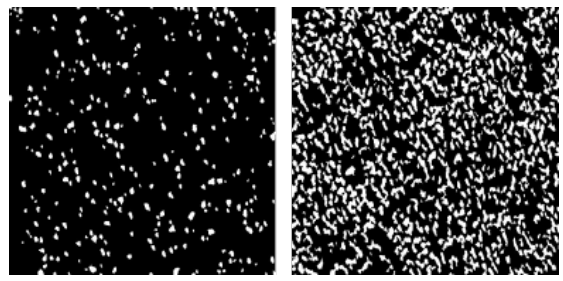

(b) Star PS

Figure 4.24: Composition of obtained nanostructures mediated by (a) linear polystyrene and (b) star shaped polystyrene. Both samples had the same molar mass $\left(\bar{M}_{\mathrm{n}}=60 \mathrm{~kg} \mathrm{~mol}^{-1}\right)$ and the same reduced grafting density $(\Sigma=1.0)$ Separation of each regime was conducted using calculated circularities. Left masks correspond to spherical micelles and right masks to worm-like micelles. (Corresponding AFM images can be found in Appendix A in Figure A1 and A3).

Images in 4.24 (a) show the isolated regimes, that were formed after dewetting the surface covered with linear polystyrene. It predominantly 
formed spherical micelles with $80 \%$ of the covered surface (left image) and a small amount of worm-like micelles (right image). The AFM image of star shaped polystyrene also yielded two dominating regimes of different domains. However, the amount of spherical micelles was determined to be less then $20 \%$ of the covered surface and the worm-like micelles dominated with $79 \%$ (see Figure 4.24 (b)). Although the two substrates had the same reduced grafting density of polystyrene the star shaped polystyrene led to a higher surface coverage and consequently to another morphology.

Since star shaped polymers spread along the surface the height difference within the nanostructure was lower and had a value of $9 \mathrm{~nm}$ compared to $14.5 \mathrm{~nm}$ for linear macromolecules. Both types of domains of the different architectures had the same value of diameter of $17 \mathrm{~nm}$ but the ratio of perimeter per domain showed differences. Domains of linear polystyrene had a perimeter of $48 \mathrm{~nm}$ whereas star shaped polystyrene formed features with $93 \mathrm{~nm}$ per domain. Features formed with star shaped polymers were more flat and had a larger perimeter. The immobilization of these macromolecules with four RAFT functionalities at the terminal end of each arm led to a higher constraint of the polymer molecule. Features with more edges and corners were formed (see Figure 4.22).

This phenomenon was confirmed by further AFM image acquisition of samples with $\Sigma=1.9$. Again two different morphologies were formed. Corresponding investigations of the composition of the nanostructures are shown in Figure 4.25. Linear polystyrene formed a mixture of $40 \%$ spherical and $60 \%$ worm-like micelles whereas star shaped polystyrene formed a dense network. The possible immobilization via four arms led again to a morphology that is expanded along the surface. The height difference within the nanostructure resulted in only $7 \mathrm{~nm}$.

The twofold reduced grafting density led to an increase in the surface coverage of $30 \%$ and $50 \%$ for linear and star shaped polystyrene, respectively. This was also observed for other molar masses. The multiple anchoring of star shaped polymer led to a transition of the regimes at lower reduced grafting density $\Sigma$.

More nanostructures mediated by constrained dewetting of grafted fourarm star shaped polystyrene with $\bar{M}_{\mathrm{n}}=60 \mathrm{~kg} \mathrm{~mol}^{-1}$ were investigated by AFM and ImageJ. The corresponding images and calculated parameters are collected in appendix A in Figure A3. 


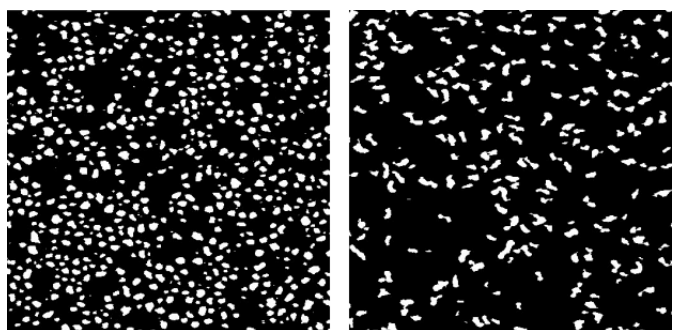

(a) Linear PS

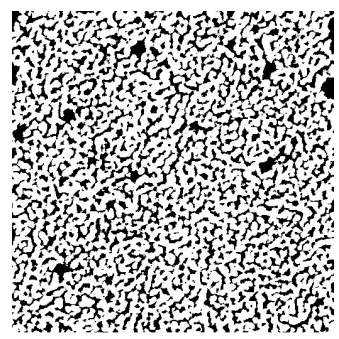

(b) Star PS

Figure 4.25: Composition of obtained nanostructures mediated by (a) linear polystyrene and (b) star shaped polystyrene. Both samples had the same molar mass $\left(\bar{M}_{\mathrm{n}}=60 \mathrm{~kg} \mathrm{~mol}^{-1}\right)$ and the same reduced grafting density $(\Sigma=1.9)$ Separation of each regime was conducted using calculated circularities. Left masks correspond to spherical micelles and right masks to worm-like micelles. (Corresponding AFM images can be found in Figure 4.16 and 4.23).

A third molar mass of $\bar{M}_{\mathbf{n}}=\mathbf{8 4} \mathbf{~ k g ~ m o l}^{-1}$ of four-arm star polystyrene was investigated. The variation of reduced grafting density led to different types of morphologies. With increasing $\Sigma$ the surface coverage increased and regimes of spherical and worm-like micelles and networks were obtained. Figure 4.26 shows AFM images of obtained nanostructures and the corresponding height histograms. All height histograms of nanostructures formed by star shaped polymers showed a monomodal distribution whereas height histograms of linear polystyrene often exhibited distributions with two maxima that clearly indicated the transition between the regimes. This crossover from one into another regime could not be observed for histograms of star shaped polystyrene. This is presumably due to the compact structure of the star polymer. The chain stretching is limited by the center and the anchoring points of the stars on the surface. Higher grafting densities rather led to fusion of the anchored polymers than to a growing in height. Thus, even low grafting densities led to network structures compared to nanostructures obtained with linear polystyrene. 
Table 4.11: Calculated reduced grafting density $\Sigma$, Euler characteristic $\chi_{\mathrm{E}}$ and domain size for morphologies resulting from constrained dewetting of star polystyrene with $\bar{M}_{\mathrm{n}}=84 \mathrm{~kg} \mathrm{~mol}^{-1}$.

\begin{tabular}{ccccccc}
\hline & $\Sigma$ & $\chi_{\mathrm{E}}$ & $\%$ area & $\begin{array}{c}\% \text { area } \\
\text { (dominat. regime) }\end{array}$ & diameter & distance \\
\hline \hline S7 & 0.3 & 710 & $15 \%$ & $9 \%$ (spherical) & $15 \mathrm{~nm}$ & $31 \pm 16 \mathrm{~nm}$ \\
S8 & 1.4 & 460 & $36 \%$ & $17 \%$ (network) & $14 \mathrm{~nm}$ & $14 \pm 6 \mathrm{~nm}$ \\
S9 & 2.9 & -60 & $46 \%$ & $38 \%$ (network) & $18 \mathrm{~nm}$ & $18 \pm 6 \mathrm{~nm}$ \\
\hline
\end{tabular}
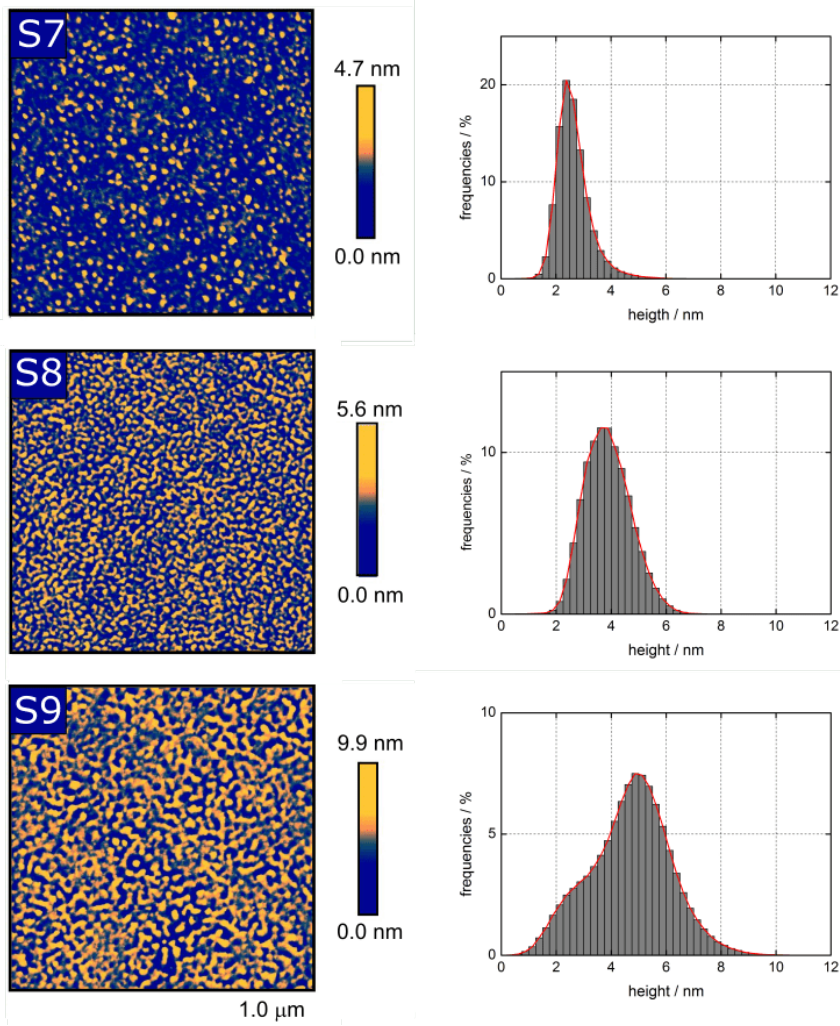

Figure 4.26: Nanostructured surfaces mediated by constrained dewetting of four-arm star polystyrene $\left(\bar{M}_{\mathbf{n}}=84 \mathbf{k g ~ m o l}^{-1}\right)$ with increasing grafting densities from S7 to S9. 


\subsubsection{Conclusion}

In the last section a quantitative analysis of atomic force microscopy images was introduced. Using the Minkowski measures, the topological Euler characteristic and geometric descriptors were calculated to describe the shape of the covered surface and the connectivity of the domains. More parameters were determined using the image processing program ImageJ, where the formed nanostructures were assigned to one of the three morphology regimes, spherical, worm-like micelles or network. The assignment was achieved via segmentation of the nanostructure by calculating the circularity of the domains.

The quantitative description was then used to analyze the formed nanostructures resulting from constrained dewetting of polystyrene. Here, two different polymer topologies were investigated: Linear and four-arm star polystyrene. Both polymer species were synthesized via RAFT polymerization and the sulphur containing RAFT group was used to anchor the polymer onto a gold substrate without prior activation or chemical modification of the substrate. The tuning of morphologies was investigated regarding the following factors:

- reduced grafting density $\Sigma$,

- molar mass,

- solvent quality during dewetting process.

For both polymer topologies, an increasing reduced grafting density led to a fusion of spherical micelles to worm-like micelles and finally to a network structure. However, for star shaped polystyrene even lower reduced grafting densities resulted in network structures compared to nanostructures obtained with linear polystyrene. This was attributed to the limited chain mobility of the branched molecules and the constraint, which results from multiple anchoring points of the star polymers on the surface.

The molar mass of the polymeric species also had a significant impact on the morphology. Low molar masses of $\bar{M}_{\mathrm{n}} \approx 20 \mathrm{~kg} \mathrm{~mol}^{-1}$ led to networks with densely populated features. For larger molar masses the obtained nanostructures varied in morphology depending on the reduced grafting density.

An advantage of constrained dewetting of homogeneous polymer layers is the reversibility of the nanostructures. It was possible to change the morphology of the nanostructure again into a homogeneous polymer layer 
and also turning it into another shape by altering the solvent quality during the dewetting process. Instead of the solvent pair dimethylformamide and water, toluene and acetone was used to dewet the polystyrene layer. The formed nanostructures tend to flatter structures with a higher surface coverage compared to the dewetting process with water. Thus, it was possible to switch between micellar and network structures for the same sample only by changing the solvent quality.

In the following section the arrangement of gold nanoparticles within the polystyrene layer is presented. The potential of polymeric nanostructures as a template to form an ordered metallic nanostructure was explored.

\subsection{Selective self-assembly of AuNPs}

\subsubsection{Preface}

Noble metal nanoparticles and their optical properties have been a fascinating topic since early centuries. ${ }^{[17]}$ In the fourth century, colloidal gold and silver nanoparticles were used to give the famous Lycurgus Cup an impressive colouring effect depending on the incidence of light. ${ }^{[118]}$ Later, metallic nanoparticles were used to give a beautiful glittering and rich colors to ceramic pottery and glass windows. The interest increased to understand this phenomenon.

First scientific investigations were conducted to understand electromagnetic and optical properties of nanoparticles. [117] Based on this research surface-enhanced Raman scattering (SERS) became a constantly increasing field of interest. [119,120] This highly sensitive spectroscopic technique enhances Raman scattering of adsorbed molecules on a nanostructured metal surface. Thus, the detection of single molecules and observation of highly informative spectra require substrates ehich ensures the right interaction of analyte and metal surface. ${ }^{[120]}$ In recent years the impact of different size and shape of noble metal nanoparticles, for instance silver and gold, was investigated. ${ }^{[121,122]}$ It was found out, that not sparse, but dense colloidal clusters of nanoparticles yield a far larger enhancement of the anti-Stokes Raman scattering of molecules. ${ }^{[117]}$ A fast and easy substrate preparation yielding the desired properties and the establishment of different substrates to increase chemical selectivity of nanostructures are nowadays the main challenges. [123] 
To generate well dispersed gold nanoparticles in any solvent, the particles need to be stabilized with specific ligand shells. Within this project, two different synthesis routes led to either citrate-stabilized gold nanoparticles in water or tetraoctylammonium bromide-capped (TOAB) gold nanoparticles dispersible in toluene. Regarding the polarity of these solvents, two potential experiments can be conducted since polystyrene shows different polymer-solvent interactions in water and toluene. First, a preformed polymeric nanostructure could serve as a template and force a certain arrangement of gold nanoparticles. Therefore, citrate-stabilized gold nanoparticles in water could be used, as the preformed nanostructure would be retained under these solvent conditions. In addition, gold substrates functionalized with four-arm star polystyrene could be immersed in a dispersion of TOABcapped gold nanoparticles in toluene. The swelling of the polystyrene layer in toluene expose free RAFT functionalities that act as a possible anchoring group to gold nanoparticles. A subsequent dewetting of these star-particle layer may also result in a metallic nanostructure.

To investigate the influence of a polymeric layer, preliminary studies of the arrangement of nanoparticles on non-functionalized gold substrates were conducted. Questions that had to be answered were:

- How does the substrate influence the nanoparticle environment?

- Which assemblies are formed without a polymeric template?

- Is a polymeric template necessary to precisely arrange nanoparticles?

Therefore, non-functionalized gold substrates were immersed in gold nanoparticle dispersions.

The obtained structures were investigated via atomic force microscopy. The depiction of the images was accomplished with the program NanoScope Analysis. The image processing program ImageJ was applied for further analysis of the nanostructures concerning the size of the obtained domains and their surface coverage.

\section{Distribution of AuNPs on non-functionalized substrates}

First, the distribution of citrate-stabilized gold nanoparticles (AuNPs) on ultraflat gold substrates without grafted polystyrene was investigated. The size and shape of metal particles were studied by transmission electron 
microscopy (TEM). A representative TEM image is shown in Appendix A in Figure A9, where well dispersed gold nanoparticles with an average diameter of $13 \mathrm{~nm}$ were obtained.

Ultraflat gold substrates were immersed in low concentrated dispersions of gold nanoparticles for different time intervals and subsequently investigated via AFM. Figure 4.27 shows the resulting structure after an immersion of one minute in a low concentrated dispersion. Even after this very short treatment, a mixed structure of separated and aggregated gold nanoparticles was obtained The two largest aggregates formed diameters of $32 \mathrm{~nm}$ and $45 \mathrm{~nm}$. Several nanoparticles attached to the gold substrate and formed an irregular structure on the metallic surface, so that particles diameters of $8 \mathrm{~nm}$ were observable.

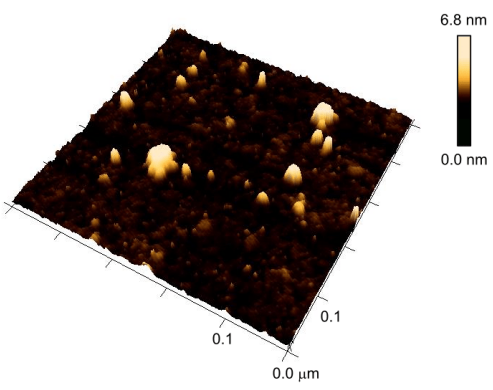

(a) Distribution after one minute.

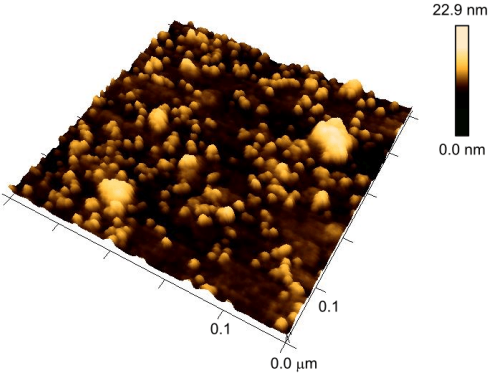

(b) Distribution after two hours.

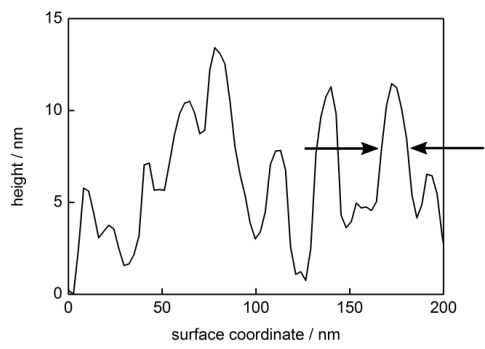

(c)

Figure 4.27: Distribution of gold nanoparticles on non-functionalized gold substrates. AFM images in (a) and (b) show the distribution of gold nanoparticles after one minute and two hours immersion, respectively. Figure 4.27 (c) shows the corresponding cross section of the structure shown in (b). 
Although, $13 \mathrm{~nm}$ sized gold nanoparticles were used, the height difference within the surface yielded only $6.8 \mathrm{~nm}$. Based on these observations, it is assumed that nanoparticle-surface interactions may distorted the stabilizing nanoparticle environment and larger agglomerations were formed. 117] Thus a structure of irregular nanoparticles with different heights, feature sizes and circularities was formed. Figure 4.27 (b) shows an AFM image of a gold substrate that was immersed in a dispersion of gold nanoparticles for two hours. The mixed structure of separated and aggregated nanoparticles was confirmed. Since a well dispersed solution of gold nanoparticles was used only homogeneously distributed nanoparticles should be obtained. However, the observation of larger aggregates on the substrate led to the assumption that the interaction between particle and substrate permits a diffusion of the nanoparticles along the surface. Consequently many gold nanoparticles may encounter each other and form aggregates.

The corresponding cross section in Figure 4.27 (c) of the AFM image shows the irregular structure formation after two hours. The formed features had different sizes and heights. Between the arrows in the middle of the peak in Figure 4.27 (c) the diameter of the particle was calculated to be approximately $(19 \pm 6) \mathrm{nm}$. From TEM measurements an average diameter of $13 \mathrm{~nm}$ was calculated. The difference can be assigned to tip artifacts during topographic imaging via atomic force microscopy. The geometrical shape of the used probe tip always has an influence on the shape of the features that have to be recorded. The height of the features is not affected whereas the lateral size is dependent on the radius of the apex and the cone angle of the tip. Figure 4.28 gives an overview about possible tip artefacts. The cone shape of the tip can cause broadening of features as it is shown on the left side. The spherical shape of attached particles can not be reproduced because of the pyramidal shape of the probe. Surfaces with holes show similar artifacts, which is demonstrated by the probe trace at the right side.
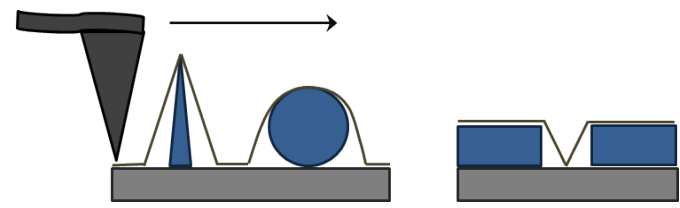

Figure 4.28: Illustration of possible tip artifacts during topographic imaging via atomic force microscopy. Through the geometrical shape of the probe tip, the lateral size of the structure can not be reproduced.. 


\subsubsection{AuNP assemblies via polymeric templates}

In the following section investigations on polymeric templates are demonstrated. A preformed polystyrene nanostructure should serve as a template to precisely arrange gold nanoparticles.

Linear polystyrene with a molar mass of $\bar{M}_{\mathrm{n}}=64 \mathrm{~kg} \mathrm{~mol}^{-1}$ was grafted onto an ultraflat gold substrate. Only substrates with low reduced grafting densities were used for dewetting experiments with dimethylformamide and water as the solvent pair. A polymeric nanostructure was formed and at the same time non-functionalized substrate was exposed. The obtained structure was used as a template and immersed in a dispersion of citratestabilized gold nanoparticles (AuNP) in water. From TEM measurements a diameter of $13 \mathrm{~nm}$ was calculated.

Figure 4.29 shows the obtained assemblies of gold nanoparticles after different functionalization intervals. From Figure 4.29 (a) to (c) AFM images in $2 \mathrm{D}$ and $3 \mathrm{D}$ of particle arrangements with increasing time of immersion are presented. After 15 minutes three percent of the surface was covered with gold nanoparticles. AFM images at the right in Figure 4.29 show the corresponding magnified 3D images. Here, the polymeric nanostructure can be observed which was retained through the whole functionalization time. The gold nanoparticles were embedded in the polymeric nanostructure. In contrast to the substrates without polymer layer, the nanoparticles were homogeneously distributed and did not change in size or geometry. Additionally, no aggregates could be obtained. Based on these observations, it can be assumed that the polymeric nanostructure stabilizes the arrangement of the nanoparticles.

After 30 minutes treatment with AuNPs the surface coverage increased to ten percent. Again, a homogeneous distribution of particles could be observed. In some regions gold nanoparticles formed clusters of several particles. However, the proximity had no influence on the symmetry of the nanoparticle environment. The longest treatment of 75 minutes yielded very dense colloidal clusters of nanoparticles where the surface coverage reached $24 \%$. The polymeric nanostructure was still observable and stabilized the particles even at close proximity.

A cross section of the covered surface after 75 minutes treatment with gold nanoparticles is shown in Figure 4.30. It becomes clear that gold nanoparticles stayed separated despite close proximity. The smaller features represent the polymeric nanostructure that stabilizes the colloidal clusters of nanoparticles. The diameter of the gold nanoparticles was determined to 

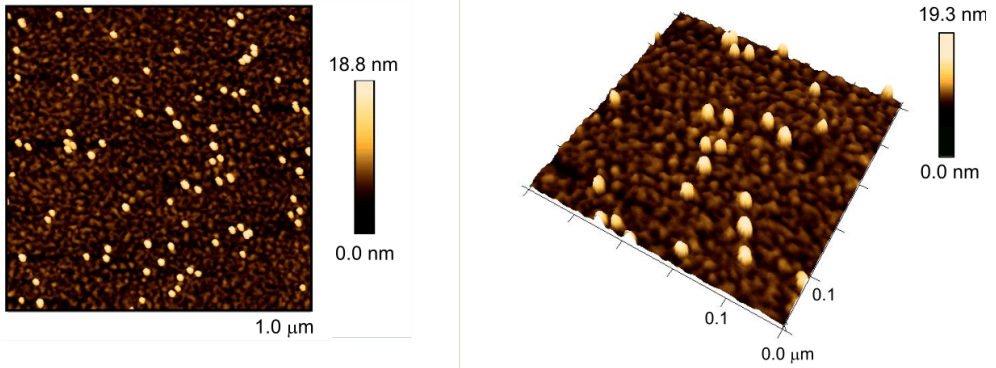

(a) 15 minutes immersion in AuNP dispersion.
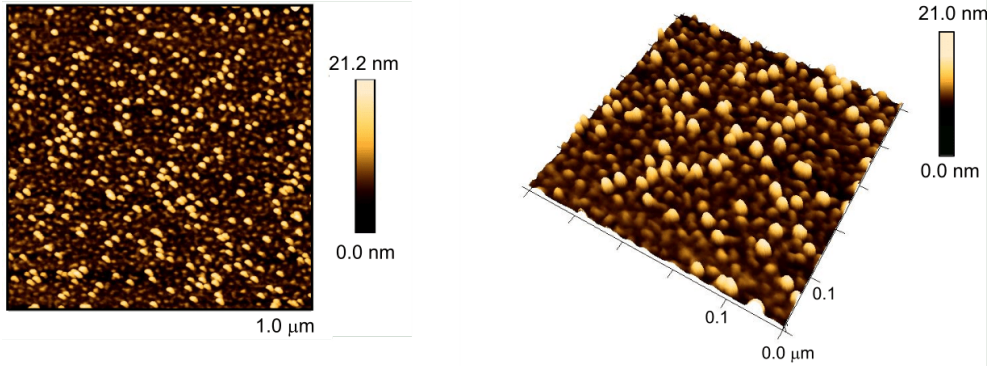

(b) 30 minutes immersion in AuNP dispersion.
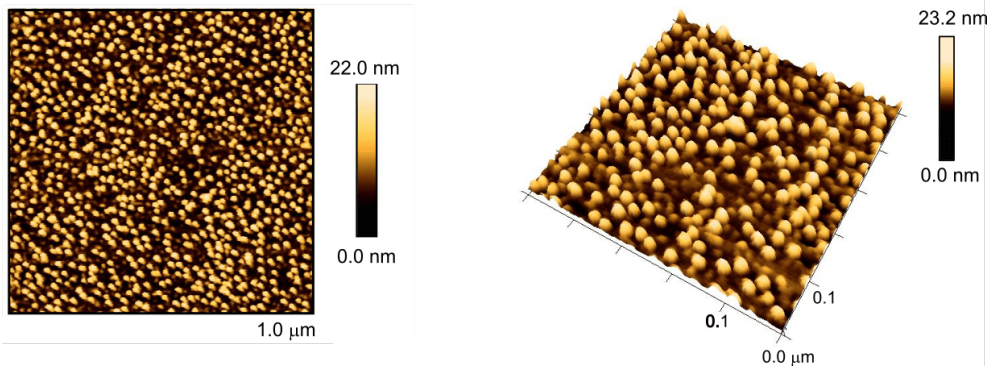

(c) 75 minutes immersion in AuNP dispersion.

Figure 4.29: AFM images in 2D at the left and corresponding magnified 3D AFM images at the right. Preformed polystyrene nanostructures on gold substrates were immersed in a dispersion of citrate-stabilized gold nanoparticles in water. From (a) to (c) the functionalization time was increasing. 
be $(17 \pm 3) \mathrm{nm}$. The difference to $d=13 \mathrm{~nm}$ determined via TEM measurements can again be attributed to tip artifacts during AFM imaging. However, compared to $d=(19 \pm 6) \mathrm{nm}$ from non-functionalized substrates the average diameter decreased and showed a smaller error interval. This fact confirms the stabilizing effect of polystyrene which prevents the gold nanoparticles to lose the spherical geometry on the one hand and aggregation on the other hand.

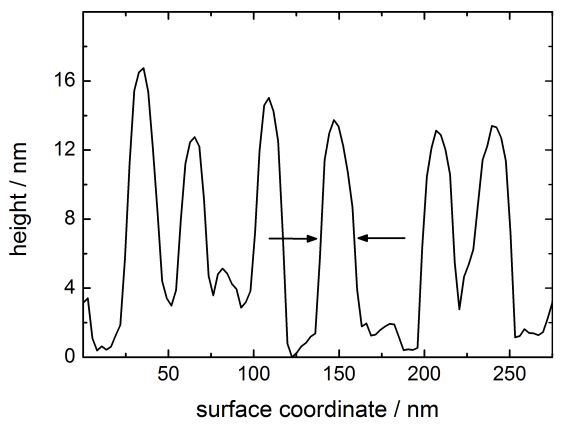

Figure 4.30: Cross section of a gold substrate with immobilized polystyrene nanostructure, which was immersed in a citrate-stabilized dispersion of gold nanoparticles in water. The functionalization time was 75 minutes. Diameter of gold nanoparticles was determined to be $(17 \pm 3) \mathrm{nm}$, which is indicated by the arrows.

\subsubsection{Tunable composite nanostructures on gold surfaces}

The formation of nanostructures via constrained dewetting of a polymeric layer involves numerous advantages. Besides easy substrate preparation, a main advantage is the reversibility between a homogeneous polymer layer and the formed nanostructure. This was already presented and explained in Section 4.2 .3 for polymeric species. In this regard, reversible composite nanostructures of star shaped polystyrene and gold nanoparticles were also investigated. Furthermore, the dependence between an increasing grafting density of star shaped polystyrene within the composite material and the formed morphology was determined.

Therefore, four-arm star polystyrene with one RAFT functionality at the terminal end of each arm was grafted onto ultraflat gold substrates. Afterwards substrates were immersed in a dispersion of tetraoctylammonium 
bromide-capped (TOAB) gold nanoparticles in toluene. A significantly smaller particle diameter of $5 \mathrm{~nm}$ was chosen to retain the reversibility between the homogeneous and structured morphology. Figure 4.31 shows the transition between these two morphologies for polystyrene and a functionalization time of gold nanoparticles of six hours. Polystyrene had a molar mass of $\bar{M}_{\mathrm{n}}=84 \mathrm{~kg} \mathrm{~mol}^{-1}$ and was grafted with $\Sigma=2.9$. On the left side the AFM image of a substrate after wetting in toluene is shown. The height difference and root mean square roughness $R_{\mathrm{q}}$ were determined to be $6 \mathrm{~nm}$ and $0.85 \mathrm{~nm}(1 \times 1 \mu \mathrm{m})$, respectively. For comparison, a homogeneous polymer layer typically has a height difference of approximately $2.5 \mathrm{~nm}$ and $R_{\mathrm{q}}=0.3 \mathrm{~nm}$. Both surface parameters increased, but no periodically nanostructured surface was obtained.

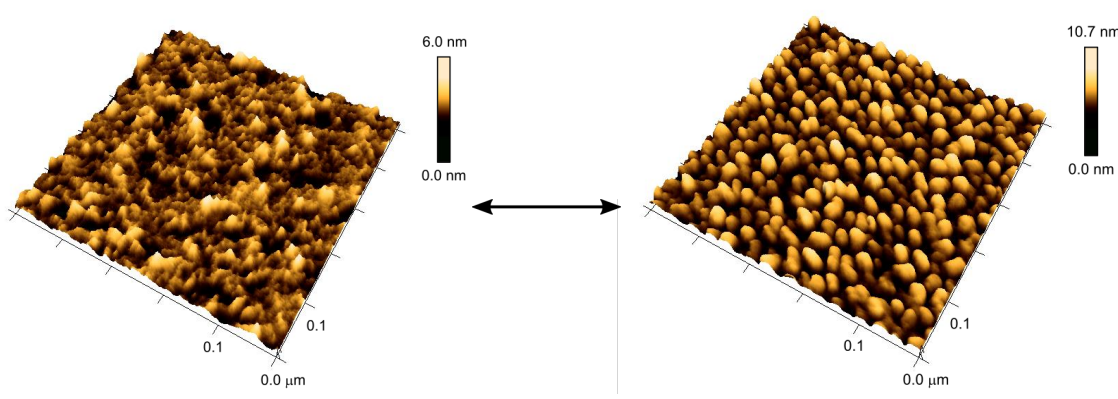

Figure 4.31: Reversibility of composite nanostructure of star shaped polystyrene ( $\bar{M}_{\mathrm{n}}=$ $84 \mathrm{~kg} \mathrm{~mol}^{-1}$ and $\left.\Sigma=2.9\right)$ and TOAB-capped gold nanoparticles $(d=5 \mathrm{~nm})$. The functionalization time was six hours. AFM images are magnified to $500 \times 500 \mathrm{~nm}$. On the left side, a representative 3D AFM image of the functionalized surface and finally immersion in toluene is shown. No periodically structured surface was obtained. After dewetting with water, a globular structure was recorded (see AFM image at the right). The surface could be reversibly switched between these two nanostructures.

This functionalized substrate was immersed in dimethylformamide and subsequently dewetted with water. An AFM image of the formed nanostructure is shown on the right side of Figure 4.31. A periodic globular nanostructure of polystyrene with gold nanoparticles was formed. The height difference and root mean square roughness within the structure increased to $10.7 \mathrm{~nm}$ and $1.71 \mathrm{~nm}(1 \times 1 \mu \mathrm{m})$, respectively. Using ImageJ the diameter of the multicomponent features was determined to be $21 \mathrm{~nm}$. Before the functionalization with gold nanoparticles the polymeric nanos- 
tructure formed a network with $d=18 \mathrm{~nm}$ after dewetting (compare sample S9 in Figure 4.26). Thus, the incorporation of gold nanoparticles changed the morphology from a network structure to a spherical symmetry with an increased diameter. A further incorporation of gold nanoparticles led to an irreversible nanostructure that is described later in this chapter.

Another composite nanostructure that can be altered by constrained dewetting is shown in Figure 4.32 . Here, a substrate with grafted fourarm star shaped polystyrene with $\bar{M}_{\mathrm{n}}=60 \mathrm{~kg} \mathrm{~mol}^{-1}$ and $\Sigma=1.9$ was immersed in a gold nanoparticle dispersion for twelve hours. The formed morphology without constrained dewetting is shown at the left side of Figure 4.32. The root mean square roughness increased to $1.19 \mathrm{~nm}(1 \times 1 \mu \mathrm{m})$ by the incorporation of nanoparticles. After constrained dewetting with dimethylformamide and water the structure turned into a morphology with spherical features. The roughness of the surface further increased to $1.67 \mathrm{~nm}$ $(1 \times 1 \mu \mathrm{m})$. The large amount of gold nanoparticles led to a high roughness for both structures and could be changed reversibly by a wetting-dewetting procedure.

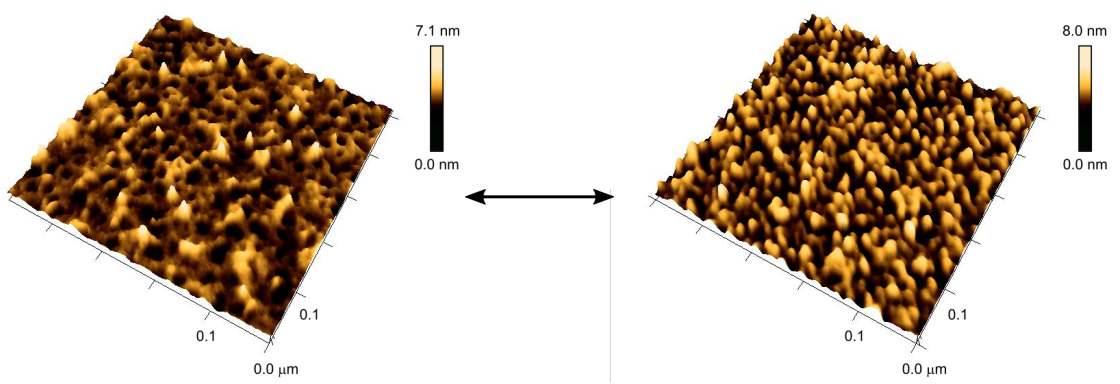

Figure 4.32: Reversibility of composite nanostructure of star shaped polystyrene $\left(\bar{M}_{\mathrm{n}}=\right.$ $60 \mathrm{~kg} \mathrm{~mol}^{-1}$ and $\left.\Sigma=1.9\right)$ and gold nanoparticles $(d=5 \mathrm{~nm})$. The functionalization time was twelve hours. AFM images are magnified to $500 \times 500 \mathrm{~nm}$. On the left side, a representative 3D AFM image of the functionalized surface and finally immersion in toluene is shown. Compared to a homogeneous surface, the root mean square roughness and height difference increased after the functionalization. After dewetting with water, a further nanostructure was recorded (see AFM image at the right). The surface could be reversibly switched between these two nanostructures. 
In summary, reversible nanostructures were obtained for high molar masses and high reduced grafting densities. Shorter chain lengths and polymer layers of low grafting density led to structures as shown in Figure 4.33. Here, a substrate with grafted star shaped polystyrene with a molar mass of $\bar{M}_{\mathrm{n}}=22 \mathrm{~kg} \mathrm{~mol}^{-1}$ and incorporated gold nanoparticles was dewetted and investigated by AFM. The nanostructure showed no difference between wetted and dewetted substrate. The chains were to short to form a structure in cooperation with gold nanoparticles.

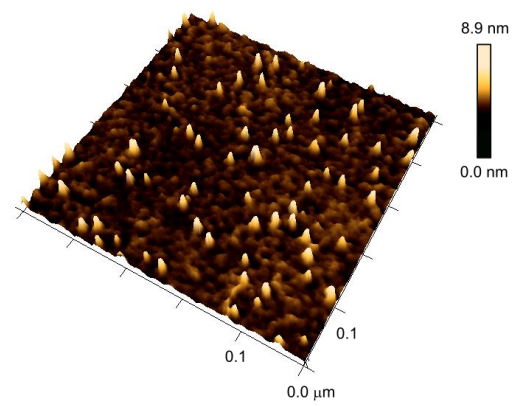

Figure 4.33: Assembly of TOAB-capped gold nanoparticles within a star shaped polystyrene layer with a molar mass of $\bar{M}_{\mathrm{n}}=22 \mathrm{~kg} \mathrm{~mol}^{-1}$. The functionalization time was four hours. AFM image is magnified to $500 \times 500 \mathrm{~nm}$. No difference between wetted and dewetted surface could be observed.

In order to fully explore the scope of this method, substrates with different reduced grafting densities of star shaped polystyrene were further exposed to a dispersion of TOAB-capped gold nanoparticles in toluene $(d=5 \mathrm{~nm})$ for an overall functionalization time of 24 hours. These substrates belong to the samples introduced in Figure 4.31 in the beginning of this section. The molar mass of the polymer was $M_{n}=84 \mathrm{~kg} \mathrm{~mol}^{-1}$. The formed nanostructures were investigated using AFM measurements and recorded AFM images are shown in Figure 4.35. The reduced grafting density of star shaped polystyrene is increasing from (a) to (c). The calculation of the Minkowski measures and the determination of the geometric characteristics using ImageJ was conducted to quantitatively analyze the depicted AFM images. The obtained values are summarized in Table 4.12. Additionally, the whole geometric analysis via ImageJ is shown in the Appendix A Figure A6. 
Table 4.12: Determined reduced grafting density $\Sigma$ of star shaped polystyrene, Euler characteristic $\chi_{\mathrm{E}}$ and domain size for morphologies with multicomponent assemblies of star shaped polystyrene with $\bar{M}_{\mathrm{n}}=84 \mathrm{~kg} \mathrm{~mol}^{-1}$ and TOAB-capped gold nanoparticles $(d=5 \mathrm{~nm})$. The functionalization time with gold nanoparticles of gold substrates with immobilized polystyrene was 24 hours

\begin{tabular}{cccc}
\hline & $\Sigma$ & $\chi_{\mathrm{E}}$ & diameter \\
\hline \hline (a) & 0.3 & 140 & $38 \mathrm{~nm}$ \\
\hline (b) & 1.4 & 210 & $\begin{array}{l}35 \mathrm{~nm} \\
15 \mathrm{~nm}\end{array}$ \\
\hline (c) & 2.9 & 540 & $21 \mathrm{~nm}$ \\
\hline
\end{tabular}

Differences of the shape and size of the domains within the formed nanostructure were even observable by visual evaluation of the depicted AFM images. The formed nanostructures show significant differences with increasing reduced grafting density. AFM images of samples with the lowest reduced grafting density of $\Sigma=0.3$ of star polystyrene showed the largest domains, that are decreasing in size with the increase in $\Sigma$. For the mean reduced grafting density of $\Sigma=1.4$ a mixed structure of small and large domains was observed. Compared to the colloidal arrangement in preformed polymeric nanostructures, that were described in Section 4.4.2. the spherical structure of the gold nanoparticles can not be observed within these structures. Based on this observation, it is assumed that domains consist of several nanoparticles incorporated in polystyrene. Additional AFM images to samples with further reduced grafting densities can be found in the Appendix in Figure A5 to clearly illustrate the observed tendency.

The observed change in domain size and shape was approved by the calculation of the Euler characteristic $\chi_{\mathrm{E}}$, that was increasing with increasing reduced grafting density. The values for $\chi_{\mathrm{E}}$ of these structures range between 140 and 540, which is compared to the values for pure polymeric nanostructures relatively small. This is expected because the obtained morpholgies could not be clearly assigned to spherical, worm-like or network structures. They did not differ in the type of the morphology, but showed differences in the size of the assemblies. The lowest reduced grafting density of polystyrene resulted in domains with the largest diameter, whereas the nanostructure with the highest reduced grafting density showed signifi- 
cantly smaller domains. The intermediate structure showed a mixture of large and small domains. This tendency was confirmed by the calculation of the diameter via ImageJ by the fragmentation in circularities. For domains with circularities tending to one, a nearly spherical geometry could be obtained, so that the calculation of the diameter was determined using the assumption of a perfect circle. For more theoretical details the reader is referred to Section 4.3. The assemblies on the substrate with a low reduced grafting density of polystyrene in picture (a), a diameter of $38 \mathrm{~nm}$ was determined. The assemblies in picture (c) showed a diameter of only $21 \mathrm{~nm}$. For the substrate with intermediate $\Sigma$ two species were obtained, larger assemblies with a diameter of $35 \mathrm{~nm}$ and smaller assemblies that showed a diameter of $15 \mathrm{~nm}$. This geometric tendency could also be observed for the cross sections of the AFM images, that are shown in Figure 4.35. The assemblies decrease in size with the increase of the reduced grafting density of polystyrene.

Based on these observations, it can be concluded that a large reduced grafting density of polymer enables a homogeneous distribution of gold nanoparticles on the surface, but limits the nanoparticle assembly in size. The surrounding polymer serves as a protective shell - the more polymer is available the smaller the aggregates. A schematic illustration is shown in Figure 4.34. The assumption of domains consisting of polymer and nanoparticle was confirmed by the measurement of quantitative nanomechanical mapping during AFM recordings. A logarithmic Derjaguin-Muller-Toporov (DMT) profile is shown in Figure A7 in Appendix A. Using a logarithmic DMT model, it can be distinguished between soft and stiff materials. This modulus showed a difference within the obtained domains, which supported the assumption of a multicomponent nature of the obtained domains.

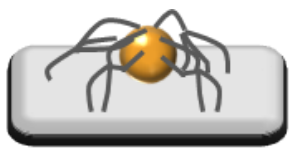

(a)

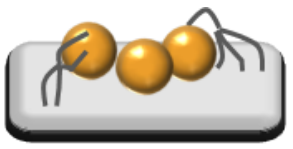

(b)

Figure 4.34: Illustration to demonstrate the formation of small and large assemblies. (a) A higher grafting density of star polystyrene can form a protective shell, thus smaller domains can be obtained. (b) A lower grafting density leads to larger domains, because of the aggregation of gold nanoparticles. 

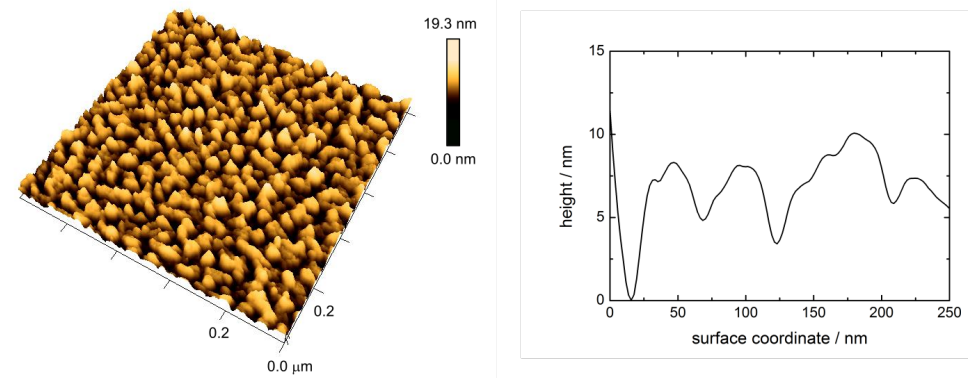

(a) Star PS with $\Sigma=0.3$ and incorporated AuNPs
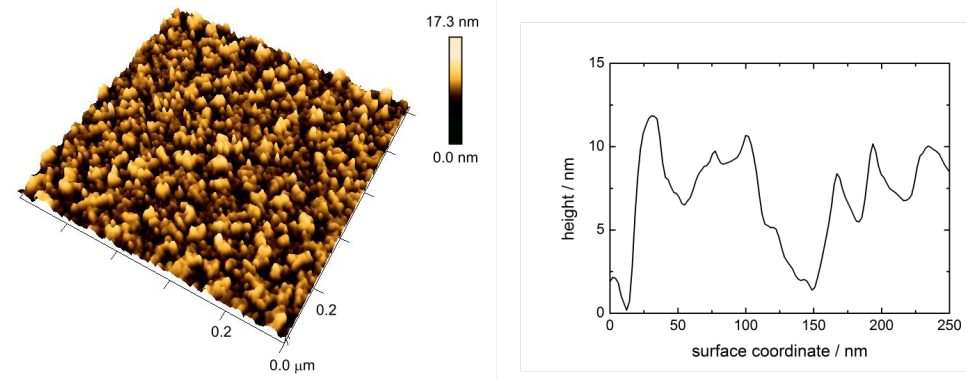

(b) Star PS with $\Sigma=1.4$ and incorporated AuNPs
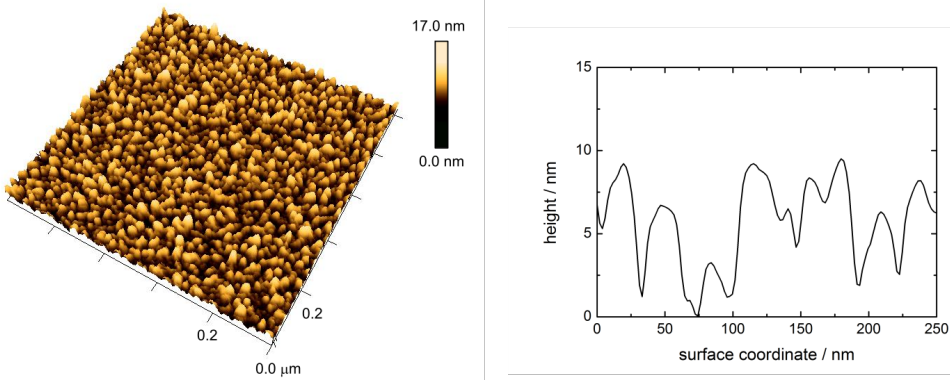

(c) Star PS with $\Sigma=2.9$ and incorporated AuNPs

Figure 4.35: Representative AFM images and corresponding cross sections of nanostructures with multicomponent assemblies of star shaped polystyrene with $\bar{M}_{\mathrm{n}}=$ $84 \mathrm{~kg} \mathrm{~mol}^{-1}$ and gold nanoparticles. The reduced grafting density of polystyrene is increasing from picture (a) to (c). 


\subsubsection{Conclusion}

In the last section the assembly of gold nanoparticles on differently functionalized gold substrates was illustrated.

First, the interaction of citrate-stabilized gold nanoparticles with nonfunctionalized substrates was investigated. Here, an irregular structure of separated nanoparticles and aggregates including random numbers of nanoparticles was formed. With increasing functionalization time the aggregates were growing. It was assumed, that the interaction between the particles and the substrate still allows a vertical diffusion of the particles along the surface. Based on this vertical diffusion, particles were able to form and grow aggregates, since a well dispersed mixture was used. A further interesting observation is the loss of spherical geometry of the gold nanoparticles. AFM images showed an irregular environment of the attached nanoparticles which was also assigned to the particle-substrate interaction.

Additionally, the influence of a polymeric template on the arrangement of gold nanoparticles on gold substrates was studied. Polystyrene was attached via its RAFT group to the surface and dewetted to form a polymeric nanostructure. The substrate was then immersed in a dispersion of citratestabilized gold nanoparticles in water, which ensures the preservation of the polymeric nanostructure. Depicted AFM images showed the arrangement of the particles into the polymeric nanostructure. With increasing functionalization time, the percentage covered surface by gold particles was increasing until they formed dense colloidal clusters. The spherical geometry was not distorted by the interaction with the gold substrate or other gold nanoparticles in proximity.

Furthermore, the arrangement of gold nanoparticles within a homogeneous polymeric layer of four-arm star polystyrene was investigated. Here, the advantage of reversibility between morphologies resulting from wetting and dewetting procedures of polymeric layers should be transferred to a composite material. For that, a gold substrate with grafted star polystyrene was immersed in a dispersion of TOAB-capped gold nanoparticles in toluene for different time intervals. For short treatments a homogeneous structure could be reversibly turned into a periodic nanostructure of polymer and gold nanoparticles. This phenomenon was particularly obtained for polymer layers with large reduced grafting densities. In order to fully explore the scope of this method, substrates with different reduced grafting densities of star shaped polystyrene were exposed to 
gold nanoparticles for a long reaction time of 24 hours. Depending on the reduced grafting density, periodic nanostructures with different size of domains were formed. It is assumed, that the formed domains consisted of several nanoparticles and polystyrene. No separated polymeric domains or separated gold nanoparticles were obtained during nanomechanical mapping. The diameter of the domains decreased with increasing reduced grafting density. This observation was assigned to a protective shell of polymer around nanoparticles that is increasing with a larger reduced grafting density and limits the aggregation of gold nanoparticles on the substrate.

\subsection{Closing remarks to Chapter 4}

In the last chapter a method to form structured polymer layers consisting of only an individual homopolymer was presented. Via constrained dewetting of thin polymer layers structured surfaces with features of several nanometers were obtained. For this purpose, polystyrene was synthesized via RAFT polymerization. This controlled method provides the opportunity to synthesize macromolecules with different architectures and at the same time end-group functionality. The RAFT group at the terminal end could be used as the anchor to gold substrates without any activation or chemical modification of the substrate. Therefore, a very easy preparation of substrates with grafted linear and star-shaped polystyrene could be established. To the best of my knowledge there is no published data that introduced an equivalent method on flat gold substrates.

First studies of the constrained dewetting of polystyrene layers demonstrated the versatility of this method. The investigations on the influence of different solvent qualities, temperature or fast and slow dewetting led to an enhanced procedure compared to ones previously published. ${ }^{[102]}$ Using this time- and labor-efficient strategy the tailoring of polymeric nanostructures by the control of the reduced grafting density was achieved. For both architectures, linear and star shaped polystyrene, three different types of morphologies could be obtained. Low reduced grafting densities yielded spherical micelles. These features grew to worm-like micelles, which then fused to a network with increasing reduced grafting density. The size of the domains could be tailored by the variation of the molar mass and polymer topology.

Major advantages of constrained dewetting of thin polymer layers were: 
- Reversibility between homogeneous and structured surface is obtained efficiently,

- Different nanopatterns were tuned by the application of different solvent qualities.

So for a substrate with a defined reduced grafting density several nanostructures were accessible and could be switched from one to another only by the immersion in different solvents.

Formed nanostructures were recorded using atomic force microscopy. The quantitative description of the AFM images was achieved with a combination of Minkowski quantities and the image processing program ImageJ. The Euler characteristic and geometric descriptors were calculated to describe the shape of the covered surface and the connectivity of the domains.

Finally, the influence of the polymer layers on the self-assembly of gold nanoparticles on gold substrates was investigated. Without grafted polymer an irregular structure of attached particles was formed, aggregates and separated ones were obtained. This result was assigned to the interaction between gold nanoparticles and gold substrate. A nanostructure of linear polystyrene was introduced to serve as a template. Without any activation of the nanostructured gold substrate, gold nanoparticles were incorporated into the nanopattern and formed dense colloidal clusters, where the spherical geometry of the particles was retained.

The advantageous characteristics of constrained dewetting and the controlled assembly of gold nanoparticles on gold surfaces could be combined. A multicomponent layer of star shaped polystyrene and gold nanoparticles could be turned into a homogeneous or structured surface reversibly depending on the surrounding solvent. With the further incorporation of gold nanoparticles, morphologies with features of different size could be developed. The domain size could be tailored by the adjustment of the reduced grafting density of the polymeric species.

Altogether, the developed constrained dewetting of stimuli-responsive thin polymer films offers a tool to form surfaces with features on the nanometer scale. The easy preparation of dense colloidal clusters of gold nanoparticles is also highly attractive for applications in fields of nanotechnology. Prominent examples are surface catalysis or the use as a substrate for surface enhanced Raman scattering. ${ }^{[117]}$ 


\section{Chapter 5}

\section{Closing remarks and outlook}

The first part of this thesis dealt with the tailored functionalization of planar surfaces with $\mathrm{pH}$-responsive polymers and their characterization via fluorescence microscopy combined with metal-induced energy transfer (MIET). This work was motivated by SFB 937. Initially, silicon substrates were modified with $\mathrm{p}$ (DMAEMA) via surface-initiated RAFT polymerization, where every reactions step was investigated in great detail. The modification was successful with regards to the controlled variation of the grafting density for different molar masses of p(DMAEMA). Then, the developed strategy was transferred to glass cover slides covered with gold and a silicon dioxide spacer. Samples of different grafting densities and molar masses were labelled with a fluorescent dye and subsequently investigated via MIET and Fluorescence correlation spectroscopy (FCS). These constitute, to the author's best knowledge, the very first successful MIET measurements of synthetic polymer chains grafted on planar substrates. Investigations in different solvent environments were conducted for all samples and their average fluorescence lifetimes were determined. Thicknesses obtained from the MIET measurements varied with the polymer grafting density in accordance with literature-based theoretical descriptions of surface-tethered polymers. When the samples were exposed to aqueous solutions at different $\mathrm{pH}$ the influence of segment-segment and polymerpolymer interactions on the calculated layer thickness could be observed. The expansion of the polymer layer led to longer fluorescence lifetimes, and consequently higher thickness values.

The MIET measurement results were verified by spectroscopic ellipsometry. Undoubtedly, the MIET method offers significantly more potential than the determination of distances of fluorophores to determine layer thicknesses. The method allows for spatial resolution on the nanometer and 
even sub-nanometer level. [124,125] As a complementary method to MIET, fluorescence correlation spectroscopy can be utilized because it is capable of monitoring lateral motions and hence provides resolution in two dimensions. The combination of both methods offers the potential to analyze dynamics of single molecules in all three dimensions.

In the frame of this work, the description of intensity fluctuations from MIET-FCS measurements of high density polymer brushes using autocorrelation functions was achieved. First results are exemplarily shown in Figure 5.1 .

Figure 5.1 (a) exhibits the entire calculated autocorrelation function. A drop in correlation indicates a process with intensity fluctuations. Figure 5.1 (b) and (c) show the region of interest of the autocorrelation function. Polymer dynamics are typically found in the $\mu$ sange. ${ }^{[53]}$ In this time range a correlation component was absent in the dry state but was observed in aqueous solution. In the dry state the fluorescent dye is incorporated in a nearly immobile polymer layer. When the polymer layer is exposed to aqueous solution the polymer chains swell and a correlation component is present. The timescale of the diffusion was $\tau=1 \cdot 10^{-6} \mathrm{~s}$ and could be attributed to the vertical diffusion of tethered polymer chain ends, since the time scale is between translational motion and rotational diffusion of free polymer chains. ${ }^{[53]}$ It has to be noted that the results show a preliminary state of the MIET-FCS studies. As can be seen in Figure 5.1 the autocorrelation function is relatively noisy. Therefore, with the data at hand it was not possible to obtain a meaningful value for the vertical diffusion time. The further improvement of the sample preparation and the analysis process are hence considered necessary to obtain a vertical diffusion time for tethered polymers in the future.

It was the goal of the project A05 of the SFB 937 to lay the experimental and theoretical groundwork for an enhanced understanding of dynamics in biological systems. The use of conventional synthetic polymers on surfaces as model systems, that were used in this work, appears to be a promising approach for this matter. A broad spectrum of monomers offers a wide scope and a cost-efficient strategy. Additionally, the effects of specific functionalities on the motion of polymer brushes can be analyzed individually. This is a distinct advantage compared to the biological models which usually exhibit high complexity. Despite considerable effort regarding the precise preparation of surface-attached polymers via RAFT polymerization, the experimental set-up yielded polymers with dispersities of 1.3 to 1.4. The use of polymers with even lower dispersities would surely 


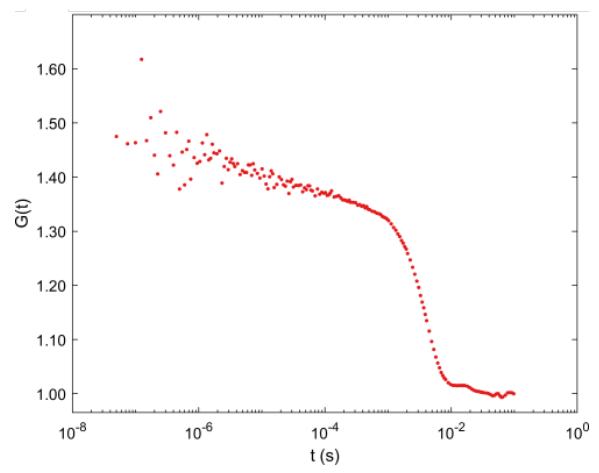

(a) autocorrelation function

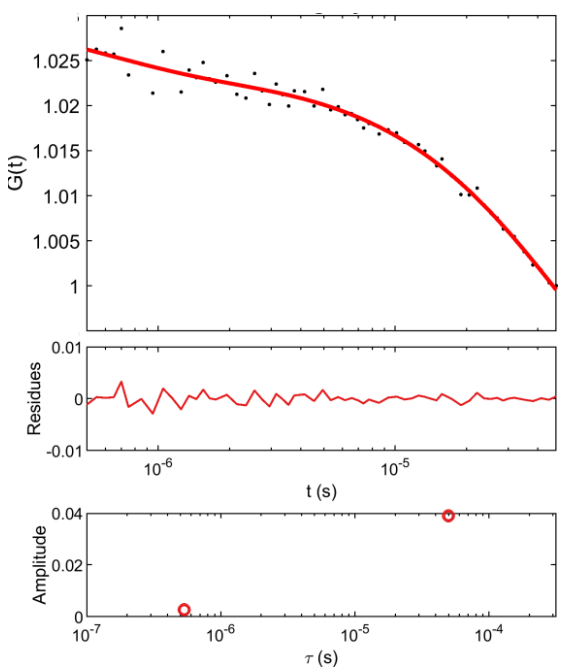

(b) dry polymer brush

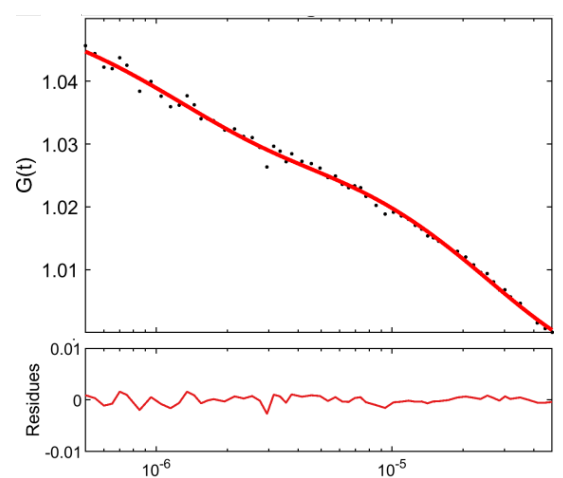

$t(s)$

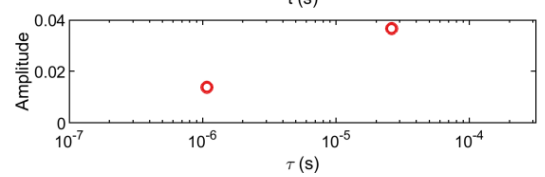

(c) polymer brush in solution

Figure 5.1: fluorescence lifetimes. Autocorrelation curves were kindly provided by Jan Christoph Thiele of the Enderlein group.

facilitate the interpretation of experimental results obtained during MIET measurements. Therefore, monomers with fewer functionalities than the utilized DMAEMA could be used as the likelihood of side-reactions during the polymerization reaction decreases, yielding better defined polymers. Since the glass transition temperature needs to be below room temperature 
to observe a cyclic motion, alkyl acrylates with longer side chains appear to be suitable candidates for future studies.

So far, only the tip of the iceberg was unveiled, showing that the interplay of FCS and MIET measurements with the preparation of suitable samples is highly demanding. The combination of the measurements of vertical and lateral diffusion to investigate a cyclic motion within a polymer brush, therefore, is considered a long-term goal that requires more interdisciplinary collaboration.

In the second part of this thesis, the scope of constrained dewetting to tailor nanostructured surfaces was explored. Linear and star shaped polystyrene was prepared via reversible addition-fragmentation chain transfer (RAFT) polymerization. The polymers were attached to gold surfaces by exploiting the affinity of the RAFT group to gold substrates. This step was achieved withoutany additional modification of the surface. Both macromolecular architectures could be utilized to produce nanostructured surfaces that ranged from spherical micelles to a network structure. The nanostructures could be tuned in their morphology by adjusting the grafting density and solvent quality. It was discovered, that they can be reversibly switched for the same sample simply by changing the solvent used in the process.

Utilizing star shaped polymers fewer polymer molecules are required to realize, for instance, network structures because of the more compact structure of star shaped polymers. Also, thinner polymeric features were achieved with star polymers because the multiple RAFT groups per macromolecule impose a stronger confinement on the polymer chains. Regarding the characterization of nanostructure morphologies, it was found that visual evaluation exclusively can be misleading. In order to achieve an unambiguous characterization, a quantitative analysis using Minkowski quantities, especially the Euler characteristic, was conducted. The quantitative analysis was completed by fragmentation of the obtained topographic images through the calculation of circularities. The classification into micelles or networks was achieved.

The potential of the obtained nanostructures was explored by the assembly of gold nanoparticles on the polymeric substrates. Compared to previously reported results, ${ }^{[102]}$ this was achieved elegantly without additional modification of the gold surface after the polymer immobilization. Since the gold nanoparticles could be synthesized in water and toluene two potential routes were offered for the assembly of nanoparticles. When gold 
nanoparticles in aqueous solution were deposited on polymeric substrates after dewetting, the gold nanoparticles were embedded into the structure and formed a nanoparticle monolayer with colloidal clusters. When gold nanoparticles in toluene were deposited on homogeneous polymer substrates, aggregates of controllable size were obtained. Their size could be controlled by the reduced grafting density of the polymer. Control over the size of nanoparticle aggregates is particularly interesting for applications in catalysis ${ }^{[8]}$ and surface-enhanced Raman spectroscopy $[105,117]$ because optimum performance is limited to specific size ranges of the particles.

For surface-enhanced Raman spectroscopy, other metals such as silver and palladium are also of particular interest. ${ }^{[105,126]}$ Expertise for the preparation of nanoparticles of both metals is already available in the Vana group [127]. It would hence be promising to investigate if control over aggregate sizes is also achievable via the established approaches for these metals. Additionally, palladium nanoparticles are an excellent candidate for reactions in surface catalysis. ${ }^{[128]}$ The polymeric nanostructures without nanoparticles are interesting for applications as microfluidic devices. [129] The introduction of photo-switchable moieties into the polymer chain might allow the development of polymeric nanostructures, whose morphology can be switched via UV-irradiation. Additionally, it might also be worthwhile to use photo-crosslinkable monomers ${ }^{[130]}$ to stabilize the obtained nanostructures reversibly against temperature and solvents.

As has been demonstrated in this work: Polymers on surfaces offer an amazing spectrum for research and applications. Small really is beautiful. 



\section{Chapter 6}

\section{Experimental section}

\subsection{Instrumental setups}

\section{Atomic force microscopy}

Atomic force microscopy (AFM) characterization was performed using a Multimode 8 AFM (Bruker) with a NanoScope V controller at ambient environment. Soft probes (ScanAsyst-Air-HR) with a nominal spring constant of $0.4 \mathrm{~N} \mathrm{~m}^{-1}$, a nominal resonance frequency of $70 \mathrm{kHz}$ and a nominal tip radius of $2 \mathrm{~nm}$ were purchased from Bruker. The ScanAsyst-HR in Air mode was used as the imaging mode with a scan rate of $0.97 \mathrm{~Hz}$ and a resolution of $512 \times 512$ samples per line. For the measurement of mechanical properties of the samples the PeakForce-Quantitative Nanomechanical Mapping (PeakForce-QNM) mode was used.

The depiction of the images was accomplished with the program NanoScope Analysis (Bruker). For the calculation of the Minkowski quantities the software Anaconda (Python distribution) was used. The specification of formed domains and the classification of the nanostructures, the image processing program ImageJ was applied.

\section{Spectroscopic ellipsometry}

The thicknesses of the thin polymer films were characterized with a spectroscopic ellipsometer Nanofilm EP4 (Accurion). Measurements were performed using nulling ellipsometry over a wavelength range of 375 to $800 \mathrm{~nm}$ with a laser stabilized xenon arc lamp with grating monochromator at an angle of incidence of light of $50^{\circ}$.

The film thickness of the silicon dioxode layer generated by air plasma on silicon substrates was determined directly after the treatment at one spot. 
The film thicknesses of $p$ DMAEMA on silicon substrates were determined by fitting the optical properties of the polymer layer with a Cauchy model. The presented values are an average of three measurements at three different spots on the substrates.

Film thicknesses of $p$ DMAEMA on silicon substrates swollen in different solvents were measured using light guides and the $5 \mathrm{x}$ objective. After the alignment in a dry state the polymer layer was exposed to the solvent for at least $15 \mathrm{~min}$. The measurement was then carried out analogous to the setup parameters in the dry state.

Polymer film thickness on glass coverslips with a gold layer were conducted at least at three different spots for statistical significance and presented as an average. To prevent back side reflection from thin transparent substrates a beam cutter was used. The characteristic optical parameters of the glass coverslip was determined using imaging ellipsometry from $385-800 \mathrm{~nm}$ at twelve points (constant energy interval) and described by a Cauchy dispersion relation $\left(A_{\mathrm{n}}=1.531, B_{\mathrm{n}}=7740 \mathrm{~nm}^{2}\right)$. The subsequent gold deposition via a titanium adhesion layer was analyzed with the same experimental procedure to obtain the optical characteristics. The dispersion for the titanium layer was taken from the database of the Accurion EP4 modelling software (referenced as from Public Sopra Database). For the gold dispersion a Drude-Lorentz-Lorentz model was applied and fitted (Drude: $A=6.849 \cdot 10^{1} \mathrm{ev}^{2}, \Gamma=0.026 \mathrm{eV}$; Lorentz 1 : Frequency $=4.844 \mathrm{eV}$, Strength $=127.350 \mathrm{ev}^{2}$, Damping $=2.340 \mathrm{eV}$; Lorentz 2 : Frequency $=2.918 \mathrm{eV}$, Strength $=7.790 \mathrm{ev}^{2}$, Damping $=0.893 \mathrm{eV}$ ). The polystyrene dispersion from Kasarova S. N. et al. was used to describe the optical characteristics of the polymer film. ${ }^{[131]}$ Spectroscopic imaging ellipsometry of the polymer films on the glass coverslips with a gold layer was performed from $400-750 \mathrm{~nm}$ at 12 points (constant energy interval). The obtained dispersion characteristics were used for the thickness calculation of all three layers.

The obtained dispersion characteristics were used for the thickness modelling of all three layers. Therefore the theoretical $\Psi \Delta$ values are calculated by a four $\times$ four Berremann algorithm and compared to the experimental spectra of $\Delta \Psi$. A levenberg marquardt routine optimizes the parameters for thickness and optical constant to minimize the deviations of theoretical and experimental data. The values of the best fit are taken for the thickness of the single layers. 


\section{Fluorescence lifetime correlation spectroscopy}

A custom-built confocal setup was used to conduct the fluorescence lifetime measurements. A $640 \mathrm{~nm}, 40 \mathrm{Mhz}$ pulsed diode laser generated the excitation light (PDL 800-B driver with LDH-D-C-640 diode, PicoQuant). The light beam was directed through a quarter-wave-plate modified to generate circular polarisation. Then, the beam was coupled into a single-mode fibre (PMC-460Si-3,0-NA012-3APC-150-P and fibre coupler 60SMS-1-4-RGBV-1147 , both Schäfter + Kirchhoff) and re-collimated by an objective after the fibre. The light beam passed a clean-up filter (UPlanSApo $10 \times 0.40$ N.A., Olympus) and subsequently a 90/10 beam splitter reflected the beam into the microscope and separated it from the emission. After directing the reflected beam into a laser scanning system (FLIMbee, PicoQuant) it was directed into a custom sideport of the microscope (Olympus IX73). Three galvo mirrors in the scanning system are imaged onto the backfocal plane of the objective (UApo N 100× 1.49 N.A. oil, Olympus) with $180 \mathrm{~mm}$ and $90 \mathrm{~mm}$ achromatic lenses. A manual xy stage (Olympus) and a z-piezo stage (Nano-ZL100, MadCityLabs) were used in order to move the sample. To collect the fluorescence in the sample, the same objective was used and the fluorescence was then descanned in the scanning system. After passing the 90/10 beam splitter, the fluorescence light was focused onto a pinhole (100 $\mu \mathrm{m}$, Thorlabs) with a $180 \mathrm{~mm}$ achromatic lens. A long-pass filter (647 LP Edge Basic, Semrock) was utilized to block backscattered laser light. A $100 \mathrm{~mm}$ lens collimated the light which was then split by a 50/50 beam spliter into two paths. Each path was passed through a bandpass filter (692/40 BrightLine, Semrock). A lens ( $f 030 \mathrm{~mm}$, Thorlabs) focused the light onto a detector ( $\tau$-SPAD, PicoQuant). The signal of the photon detector was recorded by a time-correlated single photon counting (TCSPC) system (HydraHarp 400, PicoQuant). Measurements were acquired with the SymPhoTime 64 software (PicoQuant). The software controlled the TCSPC system and the laser scanner. In a typical experiment, a pixel size of $100 \mathrm{~nm}$ was selected with a pixel dwell time of $1 \mathrm{~ms}$ and a TCSPC resolution of 16 ps.

\section{Nuclear magnetic spectroscopy}

${ }^{1} \mathrm{H}-\mathrm{NMR}$ spectra were measured with a VARIAN UNITY 300 instrument at room temperature using residual solvent proton signals as internal standard. 


\section{Plasma treatment}

Air plasma was used to clean and activate silicon substrates. It was carried out using a plasma oven Zepto from electronic Diener Plasma Surface Technology. The purification was accomplished at $100 \mathrm{~W}$ for $1000 \mathrm{~s}$.

To activate the silicon dioxide layer on glass cover slips air plasma with $20 \mathrm{~W}$ for $10 \mathrm{~s}$ was used.

\section{Size exclusion chromatography}

Size exclusion chromatography was performed with THF at $35^{\circ} \mathrm{C}$ as eluent using an AGILENT 1260 INFINITY system. The set-up comprised an isocratic HPLC-pump and an autosampler, a PSS SDV guard column $(8 \times 50 \mathrm{~mm})$ and three PSS SDV separation columns $(8 \times 300 \mathrm{~mm}$, particle size $=5 \mu \mathrm{m}$, pore sizes $=10^{3} \AA, 10^{5} \AA$ Aand $\left.10^{6} \AA\right)$. As detection system an RI detector and an $80 \mathrm{~Hz}$ UV detector that was set to a wavelength of $310 \mathrm{~nm}$ was used. The flow rate of the mobile phase was $1.0 \times 10^{-3} \mathrm{~L} \mathrm{~min}^{-1}$. Polymer samples were dissolved in THF with toluene as the internal standard and filtered through a syringe filter with a polytetrafluoroethylene (PTFE) membrane with a pore size of $0.45 \mu \mathrm{m}$. The concentration of the polymer solutions was $3 \mathrm{~g} \mathrm{~L}^{-1}$. The system was calibrated using PSS polystyrene standards of low dispersity for the measurement of polystyrene samples. For the SEC characterization of $p$ DMAEMA it was used a universal calibration using the Mark Houwink coefficients $\alpha=0.729$ and $K=0.00498 \mathrm{~mL} \mathrm{~g}^{-1}$. [132]

\section{Water contact angle}

Contact angle was measured with an OCA 15EC (OCA Measuring Instruments) via the TBO Video-based contact angle measuring system by Dataphysics. A drop of nanopure water $(2 \mu \mathrm{L})$ was placed at five different spots on the surfaces. The presented values are an average of the measurements.

\subsection{Materials}

\section{Commercially acquired substances}

The used solvents acetone, dichloromethane, dimethylformamide, ethanol, methanol, tetrahydrofuran, and toluene were purchased in pro analysi grade 
and used as received.

Anhydrous dichlormethane (МЕRCK), 3-aminopropyldimethylethoxysilane (ABCR), Atto 655-maleimide (MercK), butylamine (Alfa Aesar), 4-cyano4-[(dodecylsulfanylthiocarbonyl)sulfanyl]pentanoic acid (ABCR), hydrogen tetrachloroaurate trihydrate $\mathrm{ABCR}$ ), 2-mercapto-2-thiazoline (МERCK), $N, N^{\prime}$-dicyclohexylcarbodiimide (MERCK), propyldimethoxysilane (ABCR), sodium borohydride (MERCK), tetra- $N$-octylammonium bromide (ABCR), tris(2-carboxyethyl)phosphine hydrochloride (ABCR), (dimethylamino)pyridine (MERCK) were purchased in the highest purity available and used as received.

Magnesium sulfate (99\%, Grüssing) and potassium hydroxid pellets ( $\geq 85 \%$, MERcK) were used as received.

Nanopure (type I) water was obtained using a Millipore filtration system equipped with a UV lamp (electric resistivity was over $18.2 \mathrm{M} \Omega \mathrm{cm}$ ) and filtered through a $0.05 \mu \mathrm{m}$ cellulose filter before usage.

\section{Monomers}

Monomers used in this work were 2-(dimethylamino)ethyl methacrylate (DMAEMA) and styrene, which are shown in Figure 6.1. All monomers were freshly purified from inhibitor prior to use. DMAEMA (99\%, MERCK) was passed through a column of inhibitor remover (МеRсK). Styrene was passed through a column of basic aluminum oxide (basic, Brockmann I, 150 mesh, Merck) before use.

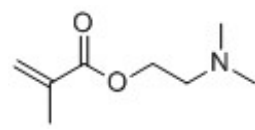

(a) DMAEMA

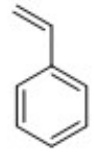

(b) Styrene

Figure 6.1: Investigated monomers within this project.

\subsection{Substrate preparation}

\section{Silicon wafer}

Silicon wafer were sliced into pieces of $1 \times 1 \mathrm{~cm}$, washed three times with aceton and simultaneously sonicated in an ultrasonication bath for three 
minutes. The substrates were blow dried using argon and finally purified and activated by air plasma for $1000 \mathrm{~s}$ and $100 \mathrm{~W}$. The height of the generated silicon dioxide layer was measured by nulling ellipsometry before usage.

\section{Glass coverslips with gold nanolayer}

Glass coverslips (Menzel, thickness $150 \mu \mathrm{m}$, refractive index 1.52) were placed in a solution of $10 \mathrm{~g}$ potassium hydroxide pellets in $50 \mathrm{~mL}$ of water and $150 \mathrm{~mL}$ ethanol and simultaneously treated with ultrasonication for $10 \mathrm{~min}$. Afterwards the glass coverslips were washed three times with nanopure water and treated with ultrasonication for $5 \mathrm{~min}$. The substrates were blow dried using nitrogen and finally treated with air plasma. The cleaned substrates were used for vapor deposition of $2 \mathrm{~nm}$ titanium and $10 \mathrm{~nm}$ of gold if not mentioned otherwise. A thin film of $30 \mathrm{~nm}$ silicon dioxide as a spacer for dynamic MIET measurements was also generated by vapor depostion. The evaporation of every layer was carried out under high-vacuum conditions $\left(\sim 10^{-6} \mathrm{mbar}\right)$ by using an electron beam source (Univex 350, Leybold). As not otherwise mentioned a depostion rate of $1 \AA \mathrm{s}^{-1}$ was used to ensure maximum smoothness on the surface. The thickness of the layers was monitored by using an oscillating quartz unit during deposition. The substrates were directly used or stored in a glove box.

\subsection{Syntheses}

\subsubsection{Activation of RAFT agent 4-cyano-4-[(dodecylsulfanyl- thiocarbonyl)sulfanyl]pentanoic acid}

The activation of the RAFT agent 4-cyano-4-[(dodecylsulfanylthiocarbonyl)sulfanyl]pentanoic acid (CDSPA) was carried out with 2-mercaptothiazoline according to similar reaction parameters reported by Li et al.. 28]

CDSPA (1.0 g, $2.5 \mathrm{mmol}, 1.0$ eq.), 2-mercaptothiazoline ( $0.3 \mathrm{~g}, 2.5 \mathrm{mmol}$, 1.0 eq.) and $N, N^{\prime}$ - dicyclohexylcarbodiimide $(0.6 \mathrm{~g}, 3.0 \mathrm{mmol}, 1.2$ eq.) were dissolved in DCM $(10 \mathrm{~mL})$. After the slow addition of 4-dimethylaminopyridine ( $61 \mathrm{mg}, 0.5 \mathrm{mmol}, 0.2 \mathrm{eq}$.) the suspension was stirred for six hours at room temperature. The reaction mixture was filtered to remove the salt. After the removal of the solvent and a silica gel column chromatography with a hexane/ethylacetate eluent with $\mathrm{v} / \mathrm{v}=5: 4$, the activated CDSPA 


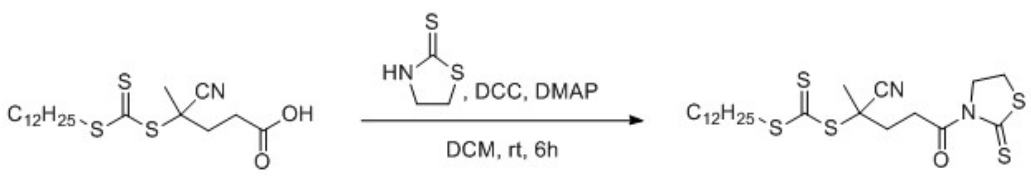

Figure 6.2: Synthesis to activate the RAFT agent.

obtained as a yellow solid (0.6 g, 48\%).

${ }^{1} \mathbf{H}$ NMR (300 MHz, $\left.\mathrm{CDCl}_{3}\right) \delta(\mathrm{ppm}): 0.88\left(\mathrm{t}, 3 \mathrm{H}, \mathrm{CH}_{3}\right) ; 1.26(\mathrm{~m}, 16 \mathrm{H}$, $\left.\mathrm{CH}_{3}-\left(\mathrm{CH}_{2}\right) 8\right) ; 1.69\left(\mathrm{~m}, 2 \mathrm{H}, \mathrm{CH}_{2}-(\mathrm{C}=\mathrm{S})\right) ; 1.89$ (s, $\left.2 \mathrm{H}, \mathrm{S}-\mathrm{C}(\mathrm{CN})\left(\mathrm{CH}_{2}\right)\right) ; 2.55$ $\left(\mathrm{m}, 2 \mathrm{H}, \mathrm{C}(=\mathrm{O})-\mathrm{CH}_{2}-\mathrm{CH}_{2}\right) ; 3.32\left(\mathrm{~m}, 2 \mathrm{H}, \mathrm{N}-\mathrm{C}(=\mathrm{S})-\mathrm{S}-\mathrm{CH}_{2}\right) ; 3.35(\mathrm{~m}, 2 \mathrm{H}$, $\left.\mathrm{S}-\mathrm{C}(=\mathrm{S})-\mathrm{S}-\mathrm{CH}_{2}\right) ; 3.57$ (m, $\left.2 \mathrm{H}, \mathrm{C}(=\mathrm{O})-\mathrm{CH}_{2}\right) ; 4.58$ (t, $\left.2 \mathrm{H}, \mathrm{C}(=\mathrm{O})-\mathrm{N}-\mathrm{CH}_{2}\right)$.

\section{Synthesis of the RAFT mask (3-Acetyltetrahydrothiazole-2-thione)}

Triethylamine $(3.26 \mathrm{~g}, 33.0 \mathrm{mmol})$ was added to a solution of 2-mercaptothiazoline $(3.0 \mathrm{~g}, 26 \mathrm{mmol})$ and THF $(60 \mathrm{~mL})$. After 15 minutes acetyle chloride $(2.28 \mathrm{~mL}, 33.0 \mathrm{mmol})$ was added and the solution was stirred for 48 hours at room temperature. The reaction was diluted by the addition of $30 \mathrm{~mL}$ $\mathrm{H}_{2} \mathrm{O}$. The reaction mixture was extracted three times with dichloromethane $(40 \mathrm{~mL})$. Afterwards the combined organic extracts were washed with a saturated solution of sodium chloride $(30 \mathrm{~mL})$ and dried using magnesium sulfate. Flash chromatography with hexane:ethyl acetate $(2: 1)$ was used as a further purification. 3-Acetyltetrahydrothiazole-2-thione was received as a yellowish oil $(2.8 \mathrm{~g}, 17 \mathrm{mmol}, 66.5 \%)$.

${ }^{1} \mathbf{H}$ NMR (300 MHz, $\left.\mathrm{CDCl}_{3}\right) \delta(\mathrm{ppm}): 2.76\left(\mathrm{~s}, 3 \mathrm{H}, \mathrm{CH}_{3}\right) ; 3.28(\mathrm{t}$, $\left.{ }^{3} J_{\mathrm{HH}}=7.5 \mathrm{~Hz}, 2 \mathrm{H}, \mathrm{NCH}_{2}\right) ; 4.56\left(\mathrm{t},{ }^{3} \mathrm{~J}_{\mathrm{HH}}=7.5 \mathrm{~Hz}, 2 \mathrm{H}, \mathrm{NCH}_{2}\right)$.

\subsubsection{SI-RAFT Polymerization from silicon substrates}

\section{Functionalization with 3-aminopropyldimethylethoxysilane}

The cleaned and activated silicon substrates with known height of the silicon dioxide layer were placed in a round bottom polymerization vial. In a general procedure in a glove box a solution of 3-aminopropyldimethylethoxysilane $(19 \mu \mathrm{L}, 0.12 \mathrm{mmol})$ in dry tetrahydrofuran $(1.5 \mathrm{~mL})$ was added to each polymerization vial. If not mentioned otherwise the substrates 
were functionalized for one hour at room temperature, afterwards rinsed with acetone three times and blow dried with argon. The functionalized substrates were directly used.

\section{Immobilization of the activated RAFT agent}

The $\mathrm{NH}_{2}$-modified surfaces were immersed in a round bottom polymerization vial with a solution of activated CDSPA $\left(4 \mathrm{mg}, 8 \cdot 10^{-6} \mathrm{~mol}\right)$ in tetrahydrofuran $(1.5 \mathrm{~mL})$. The samples were purged with argon for five minutes and left over night at room temperature. The substrates were rinsed with acetone three times and blow dried with argon. The functionalized substrates were direclty used.

\section{SI-RAFT polymerization with DMAEMA}

A polymerization mixture of DMAEMA $(1.5 \mathrm{~mL}, 8.9 \mathrm{mmol})$, activated CDSPA $\left(9.0 \mathrm{mg}, 18 \cdot 10^{-3} \mathrm{mmol}\right)$, ACCN $\left(0.9 \mathrm{mg}, 3.6 \cdot 10^{-3} \mathrm{mmol}\right)$ and dimethylformamide $(0.5 \mathrm{~mL})$ was subjected to three freeze-pump-thaw cycles to remove oxygen. The polymerizations wer carried out at $90^{\circ} \mathrm{C}$ for various time intervals. The polymerization was stopped by quenching with oxygen and cooling down with ice water. The free polymer was dissolved in acetone and precipitated in $n$-hexane to remove residual monomer. The polymer was separated by centrifugation. Two dissolving-centrifugation cycles were followed. The substrates were shaken in tetrahydrofuran for one hour to remove not attached polymer. Afterwards the surfaces were rinsed with acetone and dried with argon.

\subsubsection{Substrate preparation for dynamic MIET measurements}

The functionalization with 3-aminopropyldimethylethoxysilane (APDMES) was performed as described in the previous chapter 6.4.2. The difference in grafting density was achieved by increasing the concentration of APDMES [APDMES] $=1 \cdot 10^{-9}$ to $1 \cdot 10^{-4} \mathrm{~mol} \mathrm{~L}^{-1}$ and the functionalization time from one hour to twelve hours. For the highest grafting density the reaction mixture was additionally heated up to $80^{\circ} \mathrm{C}$.

The immobilization of the activated RAFT agent was not conducted for this substrate preparation because of the declared mechanism during the SI-RAFT polymerization at $90^{\circ} \mathrm{C}$ (see Section 3.2.3).

SI-RAFT polymerization with DMAEMA was performed as described in the previous Chapter 6.4.2. 


\section{Aminolysis of the RAFT agent}

The RAFT group of anchored $p$ (DMAEMA) on glass coverslips was removed by the immersion of the substrate in a solution of butylamine (50 $\mu \mathrm{L}$, $0.50 \mathrm{mmol}$ ) and a few crystals of tris(2-carboxyethyl)phosphine hydrochloride in $14 \mathrm{~mL}$ tetrahydrofuran over night. Tris(2-carboxyethyl)phosphine hydrochloride was added to prevent the formation of disulfide bonds. The substrate was then rinsed with copious amounts of nanopure water and aceton. The coverslips were blow dried with argon.

The aminolysis of free polymer was performed analogously. Butylamine (100 $\mu \mathrm{L}, 0.10 \mathrm{mmol}$ ) and a few crystals of tris(2-carboxyethyl)phosphine hydrochloride were added to a solution of free polymer $(0.522 \mathrm{~g}, 0.003 \mathrm{mmol})$ in $5 \mathrm{~mL}$ tetrahydrofuran. The solution was stirred over night at room temperature. The free polymer was precipitated in $n$-hexane. The polymer was separated by centrifugation. Two dissolving-centrifugation (aceton/nhexane) cycles were followed.

\section{Labeling with ATT0655 maleimide}

Aтт0655 maleimide (1 mg) was diluted to $1 \mathrm{nM}$ in anhydrous dimethylformamide and stored at $-18^{\circ} \mathrm{C} .500 \mu \mathrm{L}$ of this solution were added to a mixture of the substrate and a few crystals of tris(2-carboxyethyl)phosphine hydrochloride in $14 \mathrm{~mL}$ tetrahydrofuran. Tris(2-carboxyethyl)phosphine hydrochloride was added to prevent the formation of disulfide bonds. The reaction mixture was left in the dark for $1 \mathrm{~h}$. The substrates were rinsed with dimethylformamide and aceton to remove residual dye and finally blow dried with argon. In case the label density was too low the procedure was repeated.

The labeling of free polymer was performed analogously. Polymer with a thiol functionality at the terminal end $(0.5 \mathrm{~g})$ was dissolved in $5 \mathrm{~mL}$ dimethylformamide. After the addition of a few crystals of tris(2-carboxyethyl)phosphine hydrochloride an aliquot of the dye Atto- 655 maleimide in dimethylformamide is added. The solution is stirred for $1 \mathrm{~h}$ in the dark. The labeled polymer is precipitated in $n$-hexane. The polymer was separated by centrifugation. Two dissolving-centrifugation (aceton/ $n$-hexane) cycles were followed. 


\subsubsection{Syntheses and substrate preparation for the design of nanostructures}

\section{Homopolymerization with styrene}

Styrene polymerizations were conducted in bulk and without any initiator because the monomer is able to polymerize via thermal induced selfinitiation. To result in different molar masses two polymerization mixtures with the [M]:[RAFT] ratios [500]:[1] and [1000]:[1] were prepared. Therefore a solution of CDSPA $(90.0 \mathrm{mg}, 0.22 \mathrm{mmol})$ in styrene $(2 \mathrm{~mL}, 17.5 \mathrm{mmol})$ was prepared. Three vials with the [M]:[RAFT] ratio with [500]:[1] were made out of $390 \mu \mathrm{L}$ of the parent solution $(43.6 \mu \mathrm{mol})$ and $2.10 \mathrm{~mL}$ styrene (18.4 mmol). Another three vials with the [M]:[RAFT] ratio with [1000]:[1] were made out of $196 \mu \mathrm{L}$ of the parent solution $(21.8 \mu \mathrm{mol})$ and $2.30 \mathrm{~mL}$ styrene $(20.1 \mathrm{mmol})$. All polymerization vials were degassed with argon for at least $5 \mathrm{~min}$ and heated up to $110^{\circ} \mathrm{C}$ for $6 \mathrm{~h}, 12 \mathrm{~h}$ and $24 \mathrm{~h}$. The reaction was stopped by quenching with oxygen and cooling down with ice water. The free polymer was dissolved in dichloromethane and precipitated in ice cold methanol to remove residual monomer. The polymer was separated by centrifugation. Two dissolving-centrifugation cycles were followed.

The 4-arm star-shaped polystyrene was synthesized and kindly provided by Wentao Peng. [127

\section{Synthesis of Gold nanoparticles}

Two different kinds of gold nanoparticles (AuNPs) were used within these studies. The citrate-stabilized AuNPs were obtained as a well dispersed mixture in water. These AuNPs $\left(\beta(\mathrm{Au})=0.1 \mathrm{mg} \mathrm{mL}^{-1}\right)$ were synthesized and kindly provided by Wentao Peng. ${ }^{99]}$ The Brust-Schiffrin method gives AuNPs that can be dispersed in nonpolar solvents like toluene and were synthesized as described in the following description. [99]

A solution of hydrogen tetrachloroaurate trihydrate $(71 \mathrm{mg}, 0.18 \mathrm{mmol})$ in $15 \mathrm{~mL}$ nanopure water was added to a solution of tetra- $N$-octylammonium bromide ( $397 \mathrm{mg}, 0.73 \mathrm{mmol}$ ) in $15 \mathrm{~mL}$ toluene. After the reaction mixture was stirred vigorously for at least $5 \mathrm{~min}$ the organic phase is transferred to a new crimp-top vial. Under vigorous stirring a solution of freshly prepared sodium borohydride $(54.4 \mathrm{mg}, 1.44 \mathrm{mmol})$ in $3.75 \mathrm{~mL}$ nanopure water was added dropwise. The reaction mixture wass now stirred very 
gently for further 4 hours. The organic phase was separated and washed with dilute sulfuric acid $(5 \%, 7.5 \mathrm{~mL})$ and nanopure water ( 5 times $7.5 \mathrm{~mL}$ ) and finally dried over magnesium sulfate. The concentration of the AuNPs was estimated to be $\beta(\mathrm{Au}) \approx 1 \mathrm{mg} \mathrm{mL}^{-1}$. The AuNPs were directly used after their synthesis.

The particle size of the respective gold nanoparticles were $5 \mathrm{~nm}$ for the tetraoctylammonium bromide-capped NPs and $13 \mathrm{~nm}$ for the citratestabilized NPs. The particle size was determined using transmission electron microscopy (TEM). Measurements were done by Wentao Peng. He kindly provided the TE micrographs and size distributions that are collected in the Appendix A in Figure A9 and A8.

\section{Immobilization of RAFT-terminated polystyrene}

To control the grafting density and form a periodic nanostructure a freshly cleaned culture dish is needed. The culture dishes were placed in a solution of $10 \mathrm{~g}$ potassium hydroxide pellets in $50 \mathrm{~mL}$ of water and $150 \mathrm{~mL}$ ethanol and simultaneously treated with ultrasonication for $10 \mathrm{~min}$. Afterwards they were washed three times with nanopure water and treated with ultrasonication for $5 \mathrm{~min}$. The dishes were dried using a drying cabinet and finally treated with air plasma. Solutions of RAFT-terminated polystyrene with the concentration ranging from $2 \cdot 10^{-3} \mathrm{mg} \mathrm{mL}^{-1}$ to $2 \mathrm{mg} \mathrm{mL}^{-1}$ in toluene were freshly prepared. The glass coverslips with evaporated gold layer were then immersed in $2 \mathrm{~mL}$ of the polymer solution for various functionalization time that ranged from $10 \mathrm{~s}$ to $120 \mathrm{~s}$. Afterwards the samples were rinsed with copious amounts of toluene to remove physically attached polymer and blow dried using argon.

\section{Nanostructure formation}

The gold substrates with immobilized polystyrene were immersed in dimethylformamide in a freshly cleaned culture dish for at least 3 minutes. The wet substrates were removed and slowly exposed dropwise to nanopure water $(1 \mathrm{~mL})$. The gold substrates with nanostructured polystyrene films were gently blow dried using argon. The analysis of the formed nanostructure via AFM was conducted directly after formation. 


\section{Selective self-assembly of gold nanoparticles}

Substrates with tethered polystyrene were treated with the respective mixture of gold nanoparticles dispersed in water or toluene. If AuNPs dispersed in water are needed the mixture was used as received from the synthesis procedure $\left(\beta(\mathrm{Au}) \approx 0.1 \mathrm{mg} \mathrm{mL}^{-1}\right)$. Solutions of AuNPs in toluene were prepared at the same concentration. After the immersion in the respective AuNP solution for various times from ten minutes to 24 hours, the substrates were removed and rinsed with copious amounts of the used solvent to remove excess nanoparticles. The nanostructured surfaces were finally dried using argon. 


\section{Appendix}

\section{A. Additional figures and tables}

Table A1: Summary of determined number-average molar masses $\bar{M}_{\mathrm{n}}$ and dispersities $Ð$, which were determined via size-exclusion chromatography. Calculated polymer thicknesses from spectroscopic ellipsometry yielded the grafting density $\sigma$, which can be calculated using Equation 3.1. The abbreviations LD, MD and HD represent samples with a low, medium and high grafting density, respectively. The indices 1 and $\mathrm{h}$ indicate lower and higher number-average molar masses.

\begin{tabular}{lcccccc}
\hline & $\mathrm{LD}_{1}$ & $\mathrm{LD}_{\mathrm{h}}$ & $\mathrm{MD}_{\mathrm{l}}$ & $\mathrm{MD}_{\mathrm{h}}$ & $\mathrm{HD}_{\mathrm{l}}$ & $\mathrm{HD}_{\mathrm{h}}$ \\
\hline \hline $\bar{M}_{\mathrm{n}} / \mathrm{kg} \mathrm{mol}^{-1}$ & 13 & 36 & 23 & 36 & 25 & 36 \\
$Ð$ & 1.3 & 1.4 & 1.4 & 1.3 & 1.4 & 1.4 \\
$\sigma / \mathrm{nm}^{-2}$ & 0.09 & 0.11 & 0.19 & 0.21 & 0.39 & 0.31 \\
\hline
\end{tabular}


Further AFM images and hight histograms of nanostructures mediated by linear and star shaped polystyrene are presented. The depiction of the images was accomplished with the program NanoScope Analysis whereas the height histograms are plotted in Origin using the raw data. To give a quantitative assertion the Euler characteristic $\chi_{\mathrm{E}}$ was calculated using a python script. The image processing program ImageJ was applied for further analysis of the nanostructures like the size of the domains and their surface coverage. Calculated parameters are summarized in the respective tables.

AFM images signed with a large letter "L" concern to grafted linear polystyrene and were already discussed in the corresponding sections. The same applies to the large letter " $S$ " for four-arm star polystyrene. AFM images signed with a letter "A" are added images that are only presented in the Appendix.

Table A2: Calculated reduced grafting density $\Sigma$, Euler characteristic $\chi_{\mathrm{E}}$ and specification parameters to classify the formed nanostructures after constrained dewetting with water of linear polystyrene with $\bar{M}_{\mathrm{n}}=64 \mathrm{~kg} \mathrm{~mol}^{-1}$.

\begin{tabular}{lcccccc}
\hline & $\Sigma$ & $\chi_{\mathrm{E}}$ & $\%$ area & $\begin{array}{c}\text { \% area } \\
\text { (dominat. regime) }\end{array}$ & diameter & distance \\
\hline \hline L7 & 0.6 & 3020 & $9 \%$ & $8 \%$ (spherical) & $9 \mathrm{~nm}$ & $39 \pm 16 \mathrm{~nm}$ \\
A1 & 1.1 & 880 & $20 \%$ & $16 \%$ (spherical) & $17 \mathrm{~nm}$ & $27 \pm 10 \mathrm{~nm}$ \\
L8 & 1.9 & 940 & $29 \%$ & $18 \%$ (worm-like) & $18 \mathrm{~nm}$ & $17 \pm 8 \mathrm{~nm}$ \\
A2 & 4.3 & 390 & $36 \%$ & $19 \%$ (worm-like) & $18 \mathrm{~nm}$ & $15 \pm 7 \mathrm{~nm}$ \\
L9 & 4.4 & 350 & $34 \%$ & $17 \%$ (worm-like) & $18 \mathrm{~nm}$ & $18 \pm 9 \mathrm{~nm}$ \\
\hline
\end{tabular}



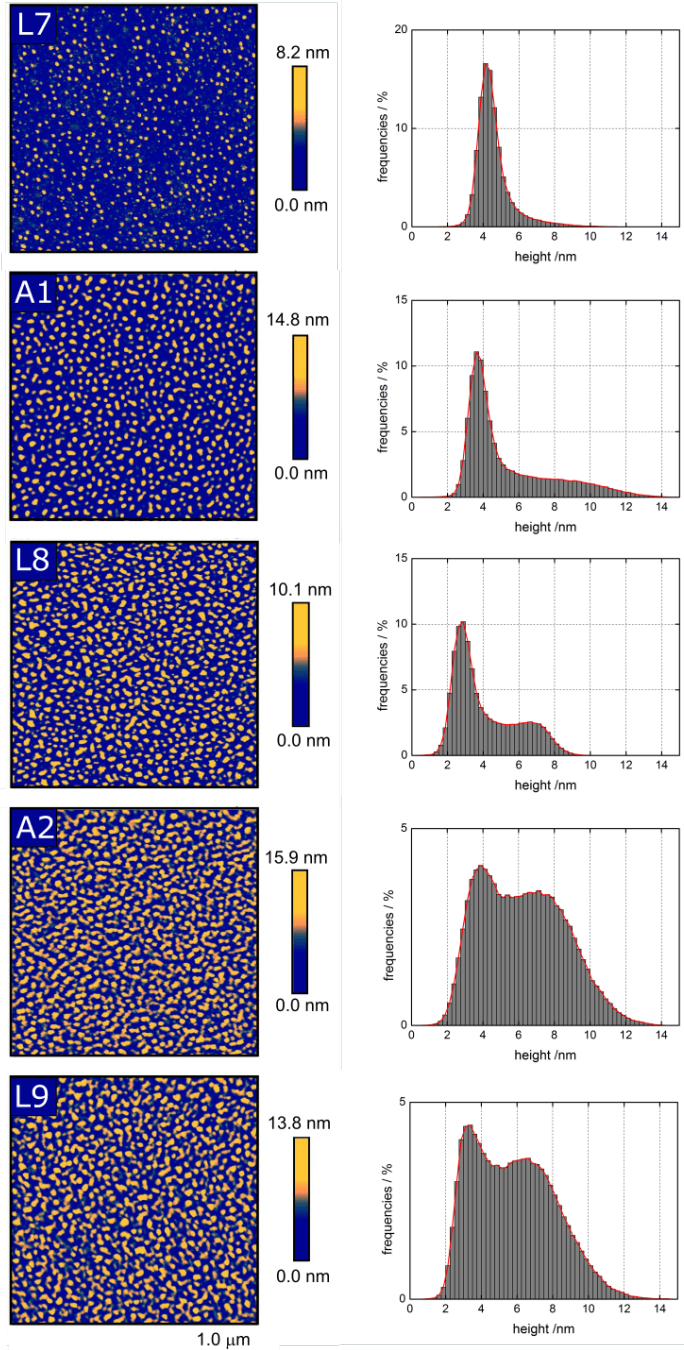

Figure A1: Representative AFM images with corresponding height histograms of nanostructures after constrained dewetting with water for linear polystyrene with $\bar{M}_{\mathrm{n}}=\mathbf{6 4} \mathrm{kg} \mathrm{mol}^{-1}$. The reduced grafting density is increasing from L7 to L9. 
Table A3: Calculated reduced grafting density $\Sigma$, Euler characteristic $\chi_{\mathrm{E}}$ and specification parameters to classify the formed nanostructures after constrained dewetting with water of four-arm star polystyrene with $\bar{M}_{\mathrm{n}}=22,60$ and $84 \mathrm{~kg} \mathrm{~mol}^{-1}$.

\begin{tabular}{|c|c|c|c|c|c|c|}
\hline & $\Sigma$ & $\chi_{\mathrm{E}}$ & $\%$ area & $\begin{array}{c}\% \text { area } \\
\text { (dominat. regime) }\end{array}$ & diameter & distance \\
\hline \multicolumn{7}{|c|}{$\bar{M}_{\mathrm{n}}=22 \mathrm{~kg} \mathrm{~mol}^{-1}$} \\
\hline A3 & 0.1 & 1000 & $48 \%$ & $39 \%$ (network) & - & - \\
\hline S1 & 0.4 & 1010 & $45 \%$ & $37 \%$ (network) & - & - \\
\hline S2 & 0.8 & 390 & $46 \%$ & $38 \%$ (network) & - & - \\
\hline S3 & 1.2 & -180 & $61 \%$ & $60 \%$ (network) & - & - \\
\hline A4 & 1.7 & 90 & $51 \%$ & $46 \%$ (network) & - & - \\
\hline \multicolumn{7}{|c|}{$\bar{M}_{\mathrm{n}}=60 \mathrm{~kg} \mathrm{~mol}^{-1}$} \\
\hline S4 & 0.1 & 1240 & $20 \%$ & 16 \% (spherical) & $14 \mathrm{~nm}$ & $21 \pm 11 \mathrm{~nm}$ \\
\hline A5 & 1.0 & 670 & $36 \%$ & 17 \% (worm-like) & $16 \mathrm{~nm}$ & $14 \pm 6 \mathrm{~nm}$ \\
\hline S5 & 1.2 & 520 & $33 \%$ & $15 \%$ (worm-like) & $17 \mathrm{~nm}$ & $16 \pm 6 \mathrm{~nm}$ \\
\hline A6 & 1.6 & 290 & $54 \%$ & $45 \%$ (network) & $18 \mathrm{~nm}$ & $11 \pm 4 \mathrm{~nm}$ \\
\hline S6 & 1.9 & 30 & $69 \%$ & $69 \%$ (network) & $18 \mathrm{~nm}$ & $9 \pm 3 \mathrm{~nm}$ \\
\hline \multicolumn{7}{|c|}{$\bar{M}_{\mathrm{n}}=84 \mathrm{~kg} \mathrm{~mol}^{-1}$} \\
\hline S7 & 0.3 & 710 & $15 \%$ & $9 \%$ (spherical) & $15 \mathrm{~nm}$ & $31 \pm 16 \mathrm{~nm}$ \\
\hline A7 & 0.9 & 780 & $32 \%$ & $17 \%$ (worm-like) & $10 \mathrm{~nm}$ & $8 \pm 4 \mathrm{~nm}$ \\
\hline S8 & 1.4 & 460 & $36 \%$ & $17 \%$ (network) & $14 \mathrm{~nm}$ & $14 \pm 6 \mathrm{~nm}$ \\
\hline A8 & 1.4 & 120 & $47 \%$ & $38 \%$ (network) & $17 \mathrm{~nm}$ & $14 \pm 5 \mathrm{~nm}$ \\
\hline S9 & 2.9 & -60 & $46 \%$ & $38 \%$ (network) & $18 \mathrm{~nm}$ & $18 \pm 6 \mathrm{~nm}$ \\
\hline
\end{tabular}



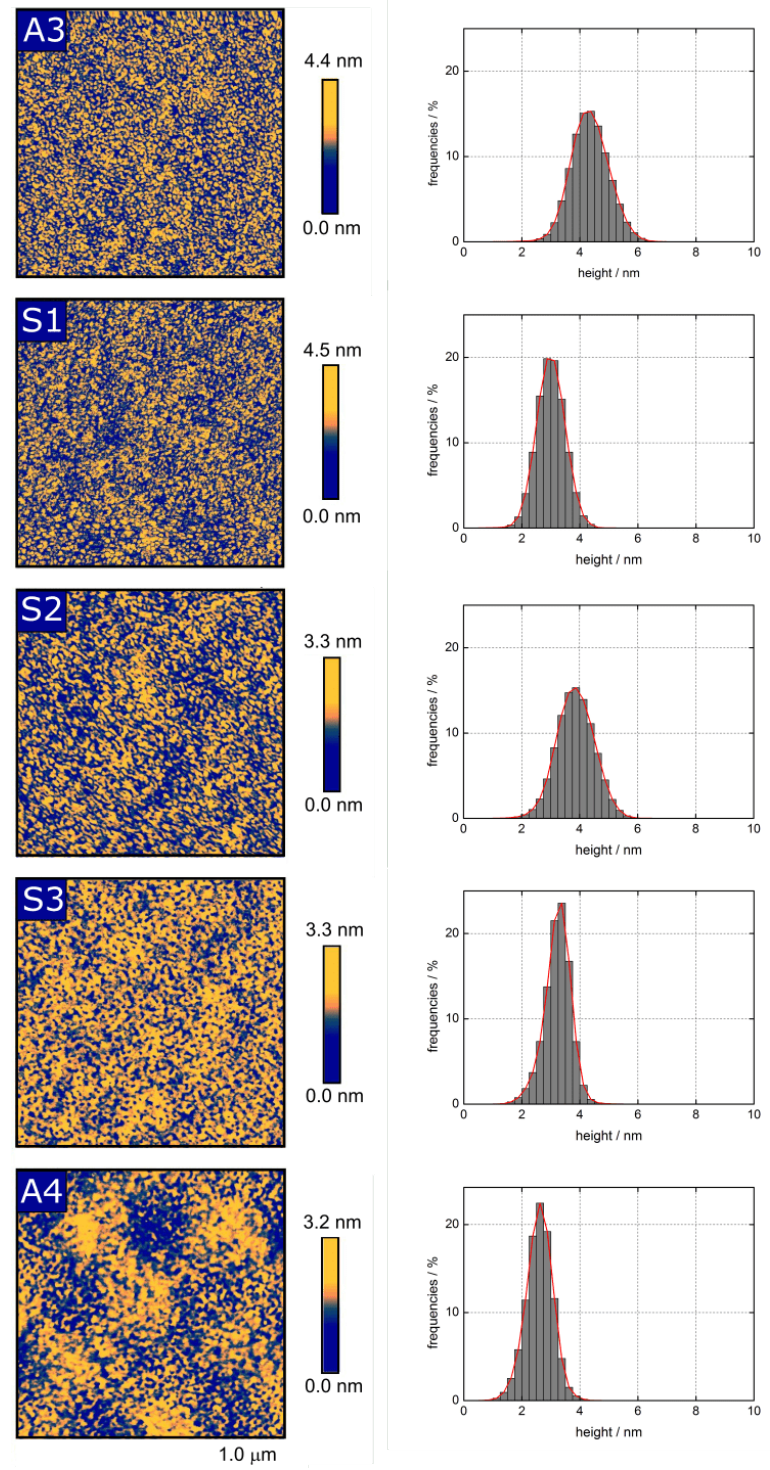

Figure A2: Representative AFM images with corresponding height histograms of nanostructures after constrained dewetting with water for four-arm star polystyrene with $\bar{M}_{\mathrm{n}}=22 \mathrm{~kg} \mathrm{~mol}^{-1}$. The reduced grafting density is increasing from S1 to A4. 

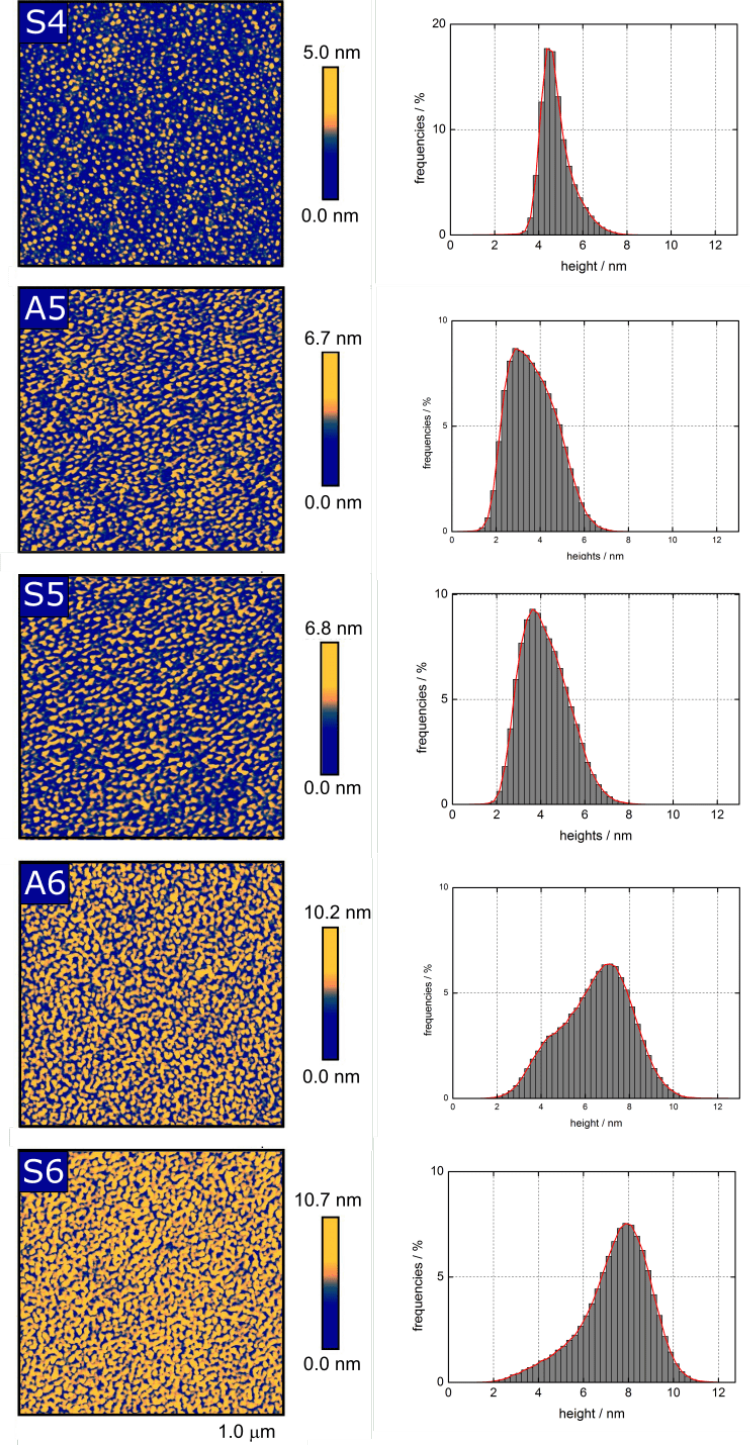

Figure A3: Representative AFM images with corresponding height histograms of nanostructures after constrained dewetting with water for four-arm star polystyrene with $\bar{M}_{\mathrm{n}}=60 \mathrm{~kg} \mathrm{~mol}^{-1}$. The reduced grafting density is increasing from S4 to S6. 

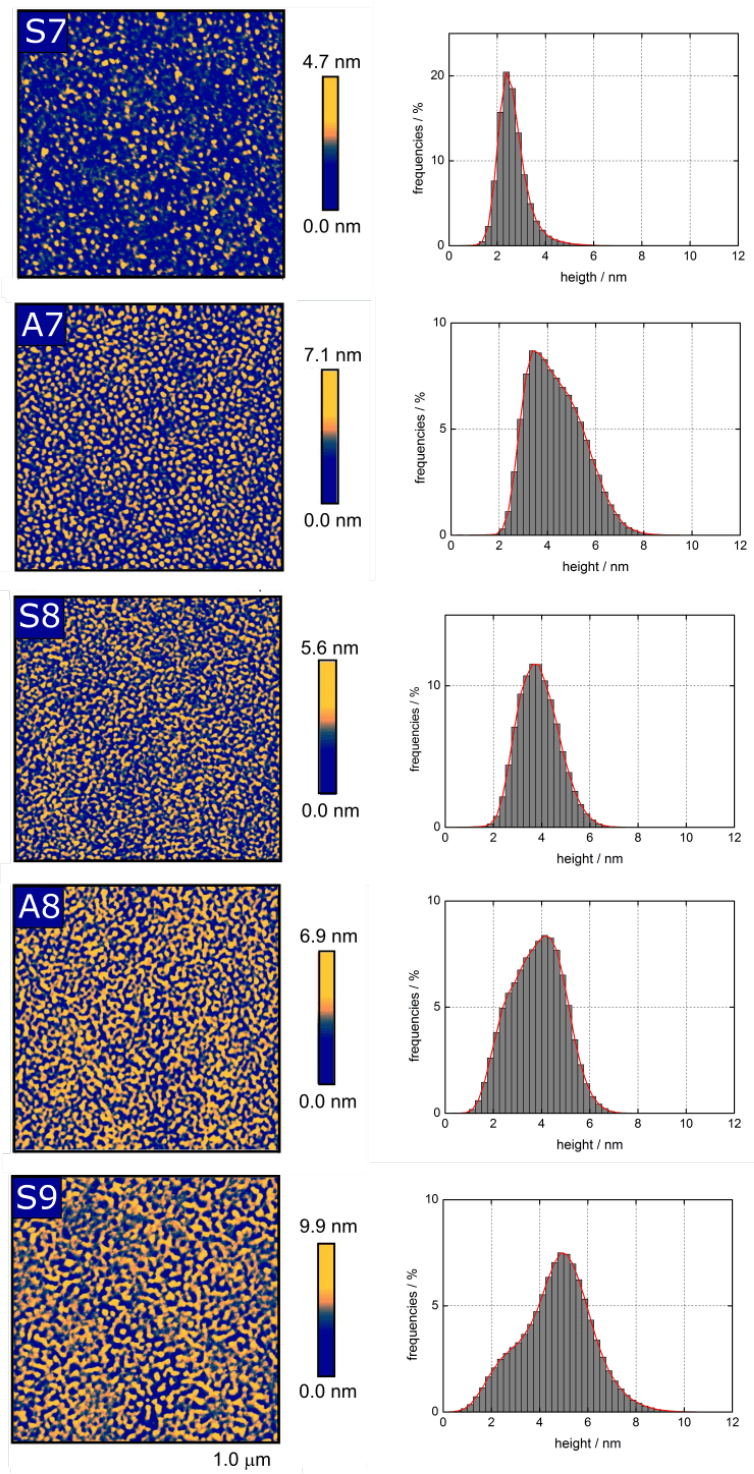

Figure A4: Representative AFM images with corresponding height histograms of nanostructures after constrained dewetting with water for four-arm star polystyrene with $\bar{M}_{\mathrm{n}}=\mathbf{8 4} \mathbf{k g ~ m o l}^{-1}$. The reduced grafting density is increasing from S7 to S9. 

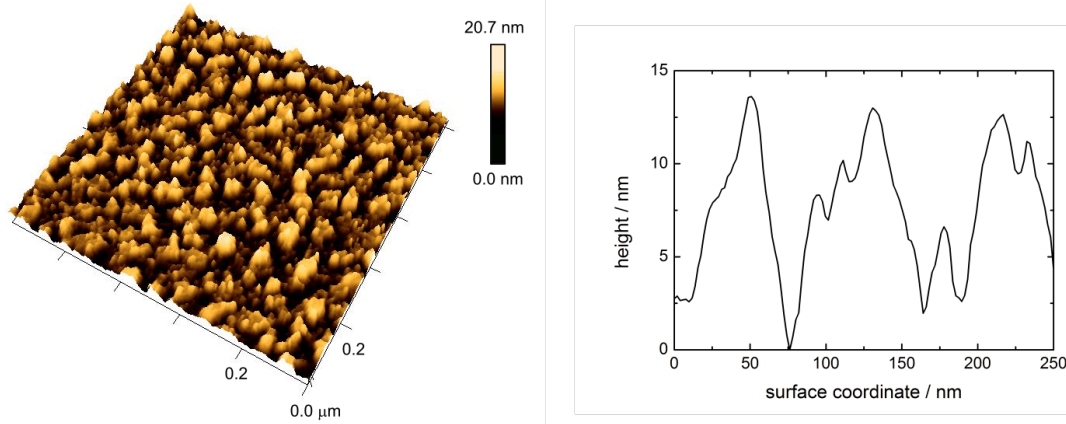

(a)
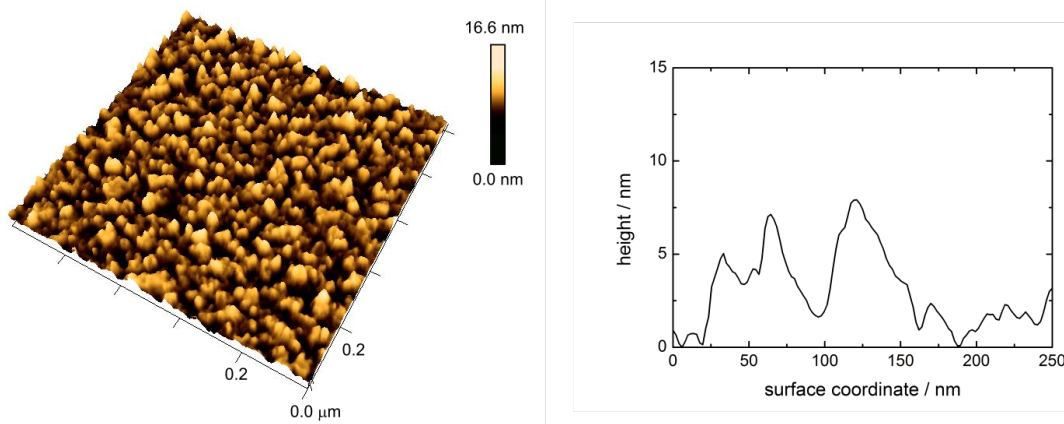

(b)

Figure A5: Representative AFM images of nanostructures with corresponding cross sections of four-arm star shaped polystyrene and gold nanoparticles on gold substrates. The molar mass of polystyrene was $\bar{M}_{\mathbf{n}}=84 \mathbf{~ k g ~ m o l}^{-1}$. The functionalization time was 24 hours. In picture (a) polymer layer had a reduced grafting density of $\Sigma=0.9$, in picture (b) $\Sigma=1.4$. 

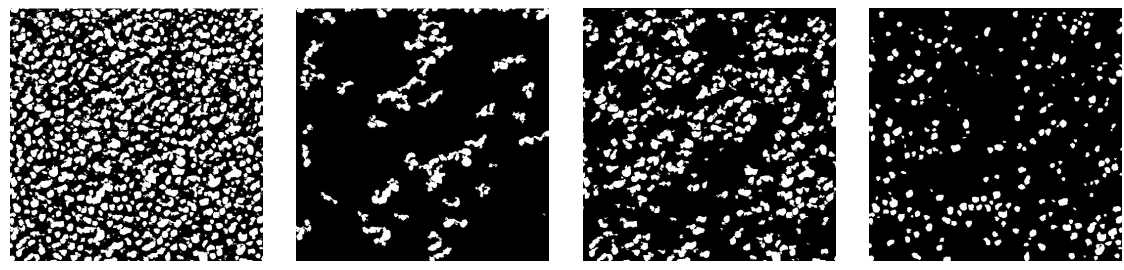

(a)
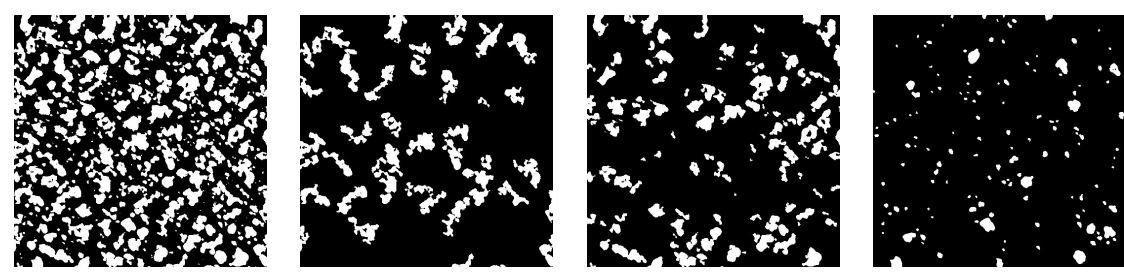

(b)
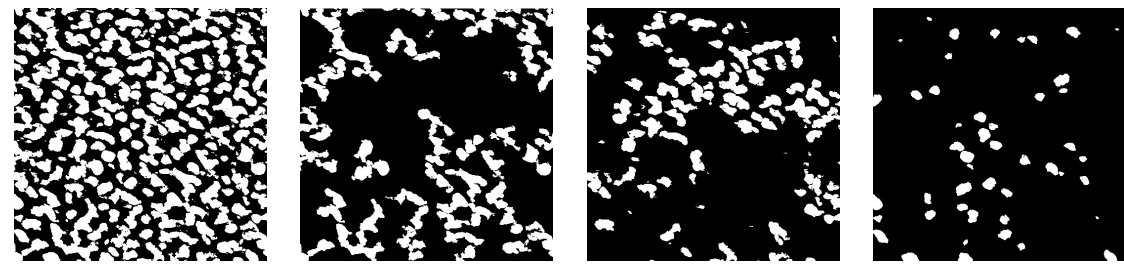

(c)

Figure A6: Fragmentation of multicomponent nanostructures by the calculation of the circularity. The structures in question are AFM images shown in Figure 4.35 in Section 3.4.3. The reduced grafting density of the polymer is increasing from (a) to (c). In one row the fragmentation from the thresholded image in network, worm-like and sperical micelles (from left to right) is shown. 

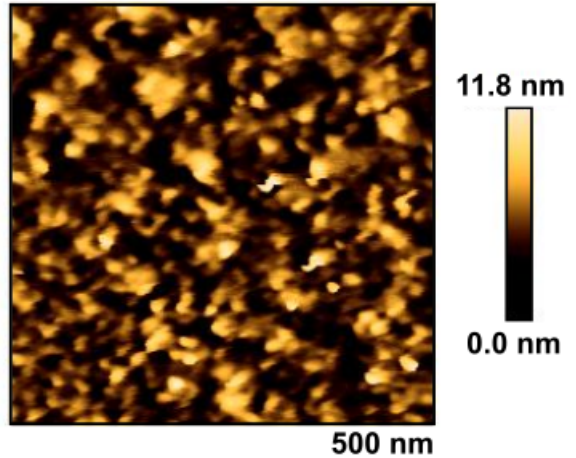

(a)

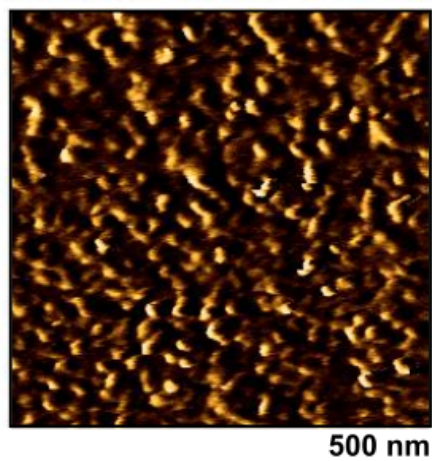

\section{6 mlog}

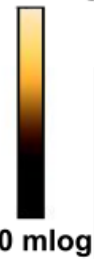

(b)

Figure A7: (a) Height AFM image of and (b) logDMT modulus image of four-arm star polystyrene with a number-average molar mass of $\bar{M}_{\mathrm{n}}=84 \mathrm{~kg} \mathrm{~mol}^{-1}$ and a reduced grafting density of $\Sigma=1.4$. TOAB-capped gold nanoparticles in toluene were incorporated into the homogeneous polymer layer. Functionalization time was 24 hours. It has to be noted, that for the logDMT modulus image, a relative scale bar is shown since no calibration to obtain absolute values was available. The light areas represent a stiff material and dark areas correspond to a softer matter. 


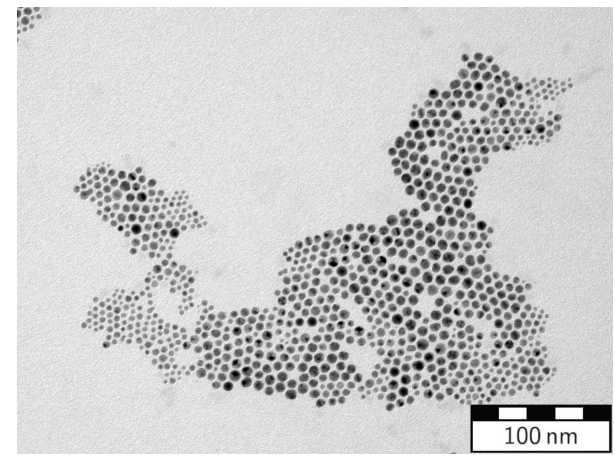

(a)

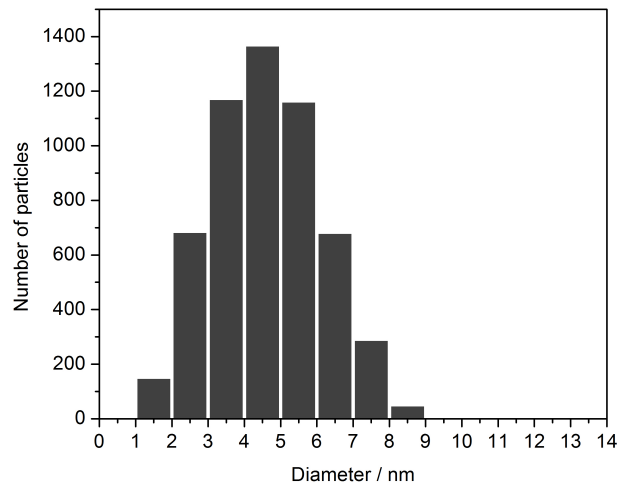

(b)

Figure A8: (a) Representative transmission electron micrograph of tetraoctylammonium bromide-capped gold nanoparticles. (b) Size distribution of tetraoctylammonium bromide-capped gold nanoparticles. 


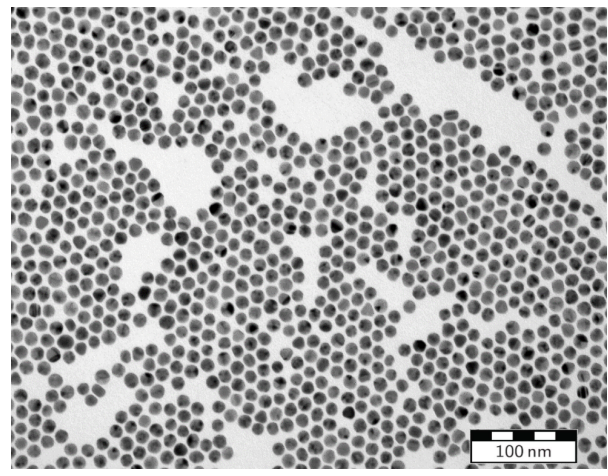

(a)

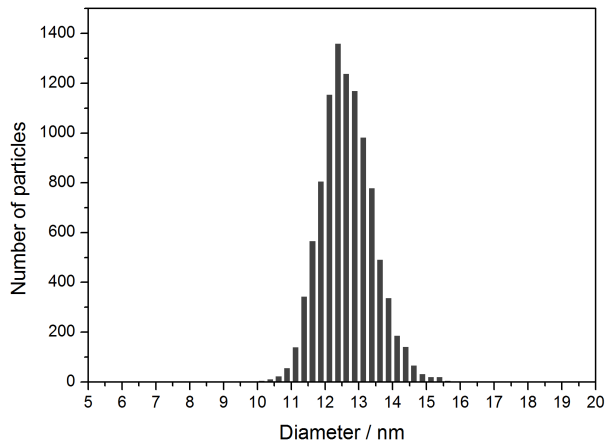

(b)

Figure A9: (a) Representative transmission electron micrograph of citrate-stabilized gold nanoparticles. (b) Size distribution of citrate-stabilized gold nanoparticles. 



\section{B. Abbreviations}

$\begin{array}{ll}\text { ACCN } & \text { 1,1'-azobis(cyclohexanecarbonitrile) } \\ \text { AFM } & \text { atomic force microscopy } \\ \text { AIBN } & \text { 2,2'-Azobis(isobutyronitrile) } \\ \text { APDMES } & \text { 3-aminopropyldimethylethoxysilane } \\ \text { AuNP } & \text { gold nanoparticle } \\ \text { CDCl }_{3} & \text { deuterated chloroform } \\ \text { CDSPA } & \text { 4-cyano-4-[(dodecylsulfanylthiocarbonyl)sulfanyl]- } \\ & \text { pentanoic acid } \\ \text { DCM } & \text { dichloromethane } \\ \text { DMAEMA } & \text { 2-(dimethylamino)ethyl methacrylate } \\ \text { DMF } & \text { dimethylformamide } \\ \text { DNA } & \text { Deoxyribonucleic acid } \\ \text { EtOH } & \text { ethanol } \\ \text { FCS } & \text { fluorescence correlation spectroscopy } \\ \text { FLCS } & \text { fluorescence lifetime correlation spectroscopy } \\ \text { FLIM } & \text { fluorescence lifetime imaging } \\ \text { FRET } & \text { Förster resonance energy transfer } \\ \text { HMA } & \text { hexyl methacrylate } \\ \text { HPLC } & \text { high-performance liquid chromatography } \\ \text { HR } & \text { high rate } \\ \text { ICS } & \text { inter-system crossing } \\ \text { m } & \text { multiplet } \\ \text { MeOH } & \text { methanol } \\ \text { MIET } & \text { metal-induced energy transfer } \\ \text { NMR } & \text { nuclear magnetic spectroscopy } \\ \text { PMA } & \text { poly(methyl acrylate) } \\ \text { PMT } & \text { photomultiplier tube } \\ \text { PS } & \text { polystyrene } \\ \text { QNM } & \text { quantitative nanomechanical mapping } \\ \text { QY } & \text { quantum yield } \\ \text { RAFT } & \text { reversible addition-fragmentation chain transfer } \\ \text { RDRP } & \text { reversible-deactivated radical polymerization } \\ \text { RI } & \text { refractive index } \\ \text { rt } & \text { room temperature } \\ \text { s } & \text { singlet } \\ \text { S } & \text { electronic singlet ground state } \\ & \end{array}$


$\mathrm{S}_{1} \quad$ electronic singlet first state

SAM self-assembled monolayer

SEC size exclusion chromatography

SERS surface-enhanced raman scattering

SI surface-induced

SPAD single-photon avalanche photodiode

St styrene

$\mathrm{t} \quad$ triplet

$\mathrm{T} \quad$ electronic triplet state

TCSPC time-correlated single photon counting

TEM transmission electron microscopy

THF tetrahydrofuran

TOAB tetraoctylammonium bromide

TS template stripping

UV ultraviolet

WCA water contact angle 



\section{Bibliography}

[1] H. Gleiter, Acta Materialia 2000, 48(1), 1-29.

[2] A. D. McFarland, C. L. Haynes, C. A. Mirkin, R. P. Van Duyne, H. A. Godwin, Journal of Chemical Education 2004, 81(4), 544A.

[3] F. Rosei, Journal of Physics Condensed Matter 2004, 16(17), 1373-1436.

[4] W. L. Chen, R. Cordero, H. Tran, C. K. Ober, Macromolecules 2017, 50(11), 4089-4113.

[5] S. Hu, X. Ren, M. Bachman, C. E. Sims, G. P. Li, N. L. Allbritton, Analytical Chemistry 2004, 76(7), 1865-1870.

[6] P. Murugan, M. Krishnamurthy, S. N. Jaisankar, D. Samanta, A. B. Mandal, Chemical Society Reviews 2015, 44(10), 3212-3243.

[7] C. Ohm, M. E. Welch, C. K. Ober, Journal of Materials Chemistry 2012, 22(37), 19343-19347.

[8] M. Koenig, D. Magerl, M. Philipp, K. J. Eichhorn, M. Müller, P. MüllerBuschbaum, M. Stamm, P. Uhlmann, RSC Advances 2014, 4(34), 17579-17586.

[9] C. Pastorino, M. Müller, Journal of Chemical Physics 2014, 140(1).

[10] M. V. D. Waarden, Journal of Colloid Science 1950, 5(4), 317-325.

[11] E. L. Mackor, J. H. van der Waals, Journal of Colloid Science 1952, 7(5), 535-550.

[12] S. Alexander, Journal de Physique 1977, 38(8), 977-981.

[13] S. Alexander, Journal de Physique 1977, 38(8), 983-987.

[14] P. G. de Gennes, Macromolecules 1980, 13(5), 1069-1075.

[15] W. J. Brittain, S. Minko, Journal of Polymer Science, Part A: Polymer Chemistry 2007, 45(16), 3505-3512.

[16] S. T. Milner, Science 1991, 251(4996), 905914. 
[17] B. Zhao, W. J. Brittain, Progress in Polymer Science (Oxford) 2000, 25(5), 677-710.

[18] M. Motornov, R. Sheparovych, E. Katz, S. Minko, ACS Nano 2008, 2(1), 41-52.

[19] O. Azzaroni, Journal of Polymer Science, Part A: Polymer Chemistry 2012, 50(16), 3225-3258.

[20] B. Ebeling, Smart Nanohybrids of RAFT Polymers and Inorganic Particles, Springer Verlag, 2015.

[21] Y. Huang, Q. Liu, X. Zhou, S. Perrier, Y. Zhao, Macromolecules 2009, 42(15), 5509-5517.

[22] C. D. Grande, M. C. Tria, M. J. Felipe, F. Zuluaga, R. Advincula, European Physical Journal E 2011, 34(2), 1-10.

[23] J. O. Zoppe, N. C. Ataman, P. Mocny, J. Wang, J. Moraes, H. A. Klok, Chemical Reviews 2017, 117(3), 1105-1318.

[24] G. Moad, E. Rizzardo, S. H. Thang, Australian Journal of Chemistry 2005, 58(6), 379-410.

[25] G. Moad, E. Rizzardo, S. H. Thang, Australian Journal of Chemistry 2006, 59(10), 669-692.

[26] G. Moad, E. Rizzardo, S. H. Thang, Australian Journal of Chemistry 2009, 62(11), 1402-1472.

[27] G. Moad, E. Rizzardo, S. H. Thang, Chemistry - An Asian Journal 2013, 8(8), 1634-1644.

[28] C. Li, B. C. Benicewicz, Macromolecules 2005, 38(14), 5929-5936.

[29] R. J. Young, P. A. Lovell, Introduction to Polymers. Third Edition, CRC Press, 2011.

[30] P. J. Flory, W. R. Krigbaum, Annual Review of Physical Chemistry 1951, 2(1), 383-402.

[31] R. S. Berry, S. A. Rice, J. Ross, Physical Chemistry. Second Edition, Oxford University Press, 2000.

[32] L. Wenning, Monte Carlo-Simulationen zum Phasenverhalten binärer Polymerbürsten, Johannes Gutenberg-Universität Mainz, 2004.

[33] G. S. Grest, M. Murat, Macromolecules 1993, 26(12), 3108-3117. 
[34] W. Zhao, G. Krausch, M. H. Rafailovich, J. Sokolov, Macromolecules 1994, 27(11), 2933-2935.

[35] T. Lee, S. C. Hendy, C. Neto, Macromolecules 2013, 46(15), 6326-6335.

[36] J. Huh, C. H. Ahn, W. H. Jo, J. N. Bright, D. R. Williams, Macromolecules 2005, 38(7), 2974-2980.

[37] K. R. Mecke, Physical Review E - Statistical Physics, Plasmas, Fluids, and Related Interdisciplinary Topics 1996, 53(5), 4794-4800.

[38] J. Wang, M. Müller, Macromolecules 2009, 42(6), 2251-2264.

[39] J. Wang, M. Müller, Journal of Physical Chemistry B 2009, 113(33), 11384-11402.

[40] J. Raczkowska, J. Rysz, A. Budkowski, J. Lekki, M. Lekka, A. Bernasik, K. Kowalski, P. Czuba, Macromolecules 2003, 36(7), 2419-2427.

[41] Handbook for nanofilm EP4, Accurion GmbH, 2016.

[42] H. G. Tompkins, E. A. Irene, Handbook of Ellipsometry, William Andrew Publishing, 2005.

[43] J. R. Lakowicz, Principles of Fluorescence Spectroscopy, Springer, 2006, 1-954.

[44] R. Heim, R. Y. Tsien, Current Biology 1996, 6(2), 178-182.

[45] W. Becker, H. Hickl, C. Zander, K. H. Drexhage, M. Sauer, S. Siebert, J. Wolfrum, Review of Scientific Instruments 1999, 70(3), 1835-1841.

[46] R. Duncan, A. Bergmann, M. A. Cousin, D. K. Apps, M. J. Shipston, Journal of Microscopy 2004, 215(1), 1-12.

[47] M. D. Eisaman, J. Fan, A. Migdall, S. V. Polyakov, Review of Scientific Instruments 2011, 82(7).

[48] D. Magde, E. Elson, W. W. Webb, Physical Review Letters 1972, 29(11), 705-708.

[49] E. L. Elson, D. Magde, Biopolymers 1974, 13, 1-27.

[50] D. Magde, E. L. Elson, W. W. Webb, Biopolymers 1974, 13, $29-61$.

[51] N. Thompson, Chemical Physics Letters 2002, 353(6), 337-378.

[52] O. Krichevsky, Reports on Progress in Physics 2002, 65, 251-297.

[53] D. Wöll, RSC Adv. 2014, 4(5), 2447-2465. 
[54] A. Ghosh, N. Karedla, J. C. Thiele, I. Gregor, J. Enderlein, Methods 2018, 140-141, 32-39.

[55] P. Kapusta, M. Wahl, M. H. J, Journal of Fluorescence 2007, 17, 43-48.

[56] I. Gregor, J. Enderlein, Photochemical and Photobiological Sciences 2007, 6, 13-18.

[57] A. I. Chizhik, J. Rother, I. Gregor, A. Janshoff, J. Enderlein, Nature Photonics 2014, 8, 124-128.

[58] T. Förster, Annalen der Physik 1948, 2(6), 55-75.

[59] W. Lukosz, R. E. Kunz, Journal of Optical Society of America 1978, 67(12), 16071615.

[60] N. Karedla, Single-Molecule Metal-Induced Energy Transfer: From Basics to Applications, Georg-August-University Göttingen, 2016.

[61] M. Szwarc, M. Levy, R. Milkovich, Journal of the American Chemical Society 1956, $78(11), 2656-2657$.

[62] D. Huebner, V. Koch, B. Ebeling, J. Mechau, J. E. Steinhoff, P. Vana, Journal of Polymer Science, Part A: Polymer Chemistry 2015, 53(1), 103-113.

[63] Y. Cai, W. Peng, S. Demeshko, J. Tian, P. Vana, Macromolecular Rapid Communications 2018, 39(13), $2-5$.

[64] G. Kissinger, W. Kissinger, Physica Status Solidi (a) 1991, 123(1), 185-192.

[65] S. Tamulevičius, I. Prosyčevas, A. Guobiene, JPuišo, Solid State Phenomena 2004, 99-100, 175-178.

[66] R. H. White, Nature 1984, 310, 430-432.

[67] T. Fukuda, A. Goto, Y. Kwak, C. Yoshikawa, Y. D. Ma, Macromolecular Symposia 2002, 182, 53-64.

[68] B. Y. Chong, J. Krstina, T. P. Le, G. Moad, A. Postma, E. Rizzardo, S. H. Thang, Macromolecules 2003, 36(7), 2256-2272.

[69] K. Skrabania, A. Miasnikova, A. M. Bivigou-Koumba, D. Zehm, A. Laschewsky, Polym. Chem. 2011, 2, 2074-2083.

[70] R. S. Faibish, W. Yoshida, Y. Cohen, Journal of Colloid and Interface Science 2002, 256(2), 341-350.

[71] E. Rakhmatullina, T. Braun, T. Kaufmann, H. Spillmann, V. Malinova, W. Meier, Macromolecular Chemistry and Physics 2007, 208(12), 1283-1293. 
[72] A. Zengin, G. Karakose, T. Caykara, European Polymer Journal 2013, 49(10), 3350-3358.

[73] S. Weinbaum, J. M. Tarbell, E. R. Damiano, Annual review of biomedical engineering 2007, 9, 121-167.

[74] K. Speyer, C. Pastorino, Soft Matter 2015, 11(27), 5473-5484.

[75] R. L. Teoh, K. B. Guice, Y. L. Loo, Macromolecules 2006, 39(25), 8609-8615.

[76] S. Mühle, M. Zhou, A. Ghosh, J. Enderlein, Physical Review E - Statistical Physics, Plasmas, Fluids, and Related Interdisciplinary Topics 2019, 100, 1-12.

[77] K. W. Vogt, P. A. Kohl, W. B. Carter, R. A. Bell, L. A. Bottomley, Surface Science 1994, 301(1-3), 203-213.

[78] M. Beversluis, A. Bouhelier, L. Novotny, Physical Review B - Condensed Matter and Materials Physics 2003, 68(11), 1-10.

[79] M. Heilemann, D. P. Herten, R. Heintzmann, C. Cremer, C. Müller, P. Tinnefeld, K. D. Weston, J. Wolfrum, M. Sauer, Analytical Chemistry 2002, 74(14), 35113517.

[80] S. T. Milner, Journal of Polymer Science Part B: Polymer Physics 1994, 32(16), 2743-2755.

[81] M. Thomas, M. Gajda, C. Amiri Naini, S. Franzka, M. Ulbricht, N. Hartmann, Langmuir 2015, 31(49), 13426-13432.

[82] M. Antonietti, Small 2016, 12(16), 2107-2114.

[83] C. Palmberg, H. Dernis, C. Miguet, OECD Science, Technology and Industry Working Papers 2009, 7.

[84] A. Botman, J. J. Mulders, C. W. Hagen, Nanotechnology 2009, 20(37).

[85] R. Courtland, IEEE Spectrum 2016, 53(9), 9-11.

[86] U. Schmelmer, A. Paul, A. Küller, M. Steenackers, A. Ulman, M. Grunze, A. Gölzhäuser, R. Jordan, Small 2007, 3(3), 459-465.

[87] S. Minko, D. Usov, E. Goreshnik, M. Stamm, Macromolecular Rapid Communications 2001, 22(3), 206-211.

[88] S. V. Orski, K. H. Fries, S. K. Sontag, J. Locklin, Journal of Materials Chemistry 2011, 21(37), 14135-14149. 
[89] H. Qiang, A. Kuller, M. Grunze, L. Junbai, Langmuir 2007, 23(7), 3981-3987.

[90] X. Liu, S. Guo, C. A. Mirkin, Angewandte Chemie - International Edition 2003, 42(39), 4785-4789.

[91] M. Kaholek, W. K. Lee, S. J. Ahn, H. Ma, K. C. Caster, B. LaMattina, S. Zauscher, Chemistry of Materials 2004, 16(19), 3688-3696.

[92] C. Slim, Y. Tran, M. M. Chehimi, N. Garraud, J. P. Roger, C. Combellas, F. Kanoufi, Chemistry of Materials 2008, 20(21), 6677-6685.

[93] S. Minko, D. Usov, C. Froeck, M. Stamm, M. Müller, A. Scholl, Physical Review Letters 2002, 88(3), 4 .

[94] B. Zhao, W. J. Brittain, W. Zhou, S. Z. Cheng, Journal of the American Chemical Society 2000, 122(10), 2407-2408.

[95] D. F. Siqueira, K. Köhler, M. Stamm, Langmuir 1995, 11(8), 3092-3096.

[96] X. Wang, X. Xiao, X. Wang, J. Zhou, N. Li, J. Xu, B. Guo, Macromolecular Rapid Communications 2007, 28(7), 828-833.

[97] A. Sidorenko, S. Minko, K. Schenk-Meuser, H. Duschner, M. Stamm, Langmuir 1999, 15(24), 8349-8355.

[98] C. Rossner, B. Ebeling, P. Vana, ACS Macro Letters 2013, 2(12), 1073-1076.

[99] C. Rossner, P. Vana Dr, Angewandte Chemie - International Edition 2014, 53(46), 12639-12642.

[100] A. S. Duwez, P. Guillet, C. Colard, J. F. Gohy, C. A. Fustin, Macromolecules 2006, 39(8), 2729-2731.

[101] I. Blakey, T. L. Schiller, Z. Merican, P. M. Fredericks, Langmuir 2010, 26(2), 692-701.

[102] M. Tebbe, E. Galati, G. C. Walker, E. Kumacheva, Small 2017, 13(37).

[103] D. Boschmann, M. Mänz, M. G. Fröhlich, G. Zifferer, P. Vana, ACS Symposium Series 2009, 1024(2), 217-232.

[104] R. Nikov, N. Nedyalkov, P. A. Atanasov, M. Terakawa, H. Shimizu, M. Obara, Applied Surface Science 2013, 264, 779-782.

[105] K. Kneipp, H. Kneipp, I. Itzkan, R. R. Dasari, M. S. Feld, Journal of Physics Condensed Matter 2002, 14(18). 
[106] N. V. Venkataraman, J. Pei, C. V. M. Cremmel, A. Rossi, N. D. Spencer, Langmuir 2013, 29(31), 9935-9943.

[107] F. Aumayr, H. Winter, Philosophical Transactions of the Royal Society A: Mathematical, Physical and Engineering Sciences 2004, 362(1814), 77-102.

[108] A. Schaub, P. Slepička, I. Kašpárková, P. Malinský, A. MacKová, V. Švorčík, Nanoscale Research Letters 2013, 8(1), 1-8.

[109] T. Smith, Journal of Colloidand Interface Science 1980, 75(1), 51-55.

[110] E. F. Casassa, Polymer Solutions: Their Modelling and Structure, 1, Oxford Univ. Press: Clarendon, 1990.

[111] J. F. Douglas, J. Roovers, K. F. Freed, Macromolecules 1990, 23(18), 4168-4180.

[112] W. W. Graessley, J. Roovers, Macromolecules 1979, 12(5), 959-965.

[113] X. Zhang, F. Shi, J. Niu, Y. Jiang, Z. Wang, Journal of Materials Chemistry 2008, 18(6), 621-633.

[114] E. Yu, S. C. Kim, H. J. Lee, K. H. Oh, M. W. Moon, Scientific Reports 2015, 5, $2-7$.

[115] R. N. Wenzel, Industrial and Engineering Chemistry 1936, 28(8), 988-994.

[116] A. Rawal, Materials Letters 2011, 65(10), 1457-1459.

[117] N. J. Halas, S. Lal, W.-s. Chang, S. Link, P. Nordlander, Chemical Reviews 2011, 111, 3913-3961.

[118] U. Leonhardt, Nature Photonics 2007, 1(4), 207-208.

[119] M. Fleischmann, P. J. Hendra, A. J. McQuillan, Chemical Physics Letters 1974, 26(2), 163-166.

[120] Y. Hu, J. Liao, D. Wang, G. Li, Analytical Chemistry 2014, 86(8), 3955-3963.

[121] J. Kneipp, H. Kneipp, K. Kneipp, Chemical Society Reviews 2008, 37(5), 10521060.

[122] J. J. Mock, M. Barbic, D. R. Smith, D. A. Schultz, S. Schultz, Journal of Chemical Physics 2002, 116(15), 6755-6759.

[123] L. Tian, S. Tadepalli, M. E. Farrell, K. K. Liu, N. Gandra, P. M. Pellegrino, S. Singamaneni, Journal of Materials Chemistry C 2014, 2(27), 5438-5446. 
[124] N. Karedla, A. I. Chizhik, I. Gregor, A. M. Chizhik, O. Schulz, J. Enderlein, ChemPhysChem 2014, 15(4), 705-711.

[125] A. Ghosh, A. Sharma, A. I. Chizhik, S. Isbaner, D. Ruhlandt, R. Tsukanov, I. Gregor, N. Karedla, J. Enderlein, Nature Photonics 2019, 13, 860-865.

[126] M. E. Abdelsalam, S. Mahajan, P. N. Bartlett, J. J. Baumberg, A. E. Rusell, Journal of the American Chemical Society 2007, 129(23), 7399-7406.

[127] W. Peng, C. Rossner, V. Roddatis, P. Vana, ACS Macro Letters 2016, 5(11), 1227-1231.

[128] S. Santoro, S. I. Kozhushkov, L. Ackermann, L. Vaccaro, Green Chemistry 2016, 18(12), 3471-3493.

[129] L. Ionov, N. Houbenov, A. Sidorenko, M. Stamm, S. Minko, Advanced Functional Materials 2006, 16(9), 1153-1160.

[130] K. Tietz, S. Finkhäuser, K. Samwer, P. Vana, Macromolecular Chemistry and Physics 2014, 215(16), 1563-1572.

[131] S. N. Kasarova, N. G. Sultanova, C. D. Ivanov, I. D. Nikolov, Optical Materials 2007, 29(11), 1481-1490.

[132] K. B. Kockler, F. Fleischhaker, C. Barner-Kowollik, Macromolecules 2016, 49(22), 8572-8580. 


\section{Acknowledgements}

I would like to thank my supervisor Prof. Dr. Philipp Vana for the opportunity to prepare my masters thesis and my PhD thesis in his research group. It was a great feeling to experience his confidence in my ability to support the success of our group. He helped me to develop an even more solution oriented mindset. I always appreciated his supporting and encouraging words during discussions.

I am grateful for my co-supervisor Prof. Dr. Marcus Müller who showed at any time deep interest in my topics. Our inspiring talks made me appreciate the passion and work of theoretical physicists - which is as hard as it seems. I always felt highly encouraged by him.

Professor Michael Buback, Professor Jörg Enderlein, Professor Burkhard Geil and Professor Andreas Janshoff are gratefully acknowledged for being part of my thesis committee. I would like to thank all my collaboration partner during my PhD studies. Dr. Sebastian Isbaner and Jan Christoph Thiele were great team members in the SFB project 937. During our collaboration we spent a lot of time together and we became also friends. Many thanks to Christoph for measuring and analysing countless samples for several hours and days. Nils Doerrer deserves special credit for compiling of the python script for the calculation of the Minkowski quantities that was exceedingly helpful. He was always patient with me and helped me without hesitation.

I would like to thank my lovely bachelor student Qingyuan Song. She is an extraordinary young student with plenty of ambition and talent. We learned a lot from each other.

I would like to thank Wentao Peng for lots of scientific exchange and providing nanoparticles and some chemicals.

I also want to thank Christian Hoffmann from Accurion $\mathrm{GmbH}$ for all his support. He was always eager to help, be it in person or via phone and made my life with the ellipsometer a lot easier. 
I enjoyed my time in the research group of Prof. Philipp Vana and I want to thank all of my current and former colleagues for the great atmosphere. I will miss the gummy bears, our funny conversations during lunch and of course the support of every single group member during the last four years. In particular, I want to thank Sonja Schmidt, Judith Steinhoff, Stella Weber, Michael Hendrich and Christian Roßner for a lot of scientific advice and carefully proof reading my doctor thesis. I would also like to thank Heike Rohmann and Sandra Lotze for being so creative to solve all of my technical problems.

I also want to thank my parents Gitta and Günter. Without Günter I would have never been able to go to Göttingen for my Chemistry studies and I am grateful for their continuous support throughout my studies.

I am deeply grateful for my phenomenal friends Jana Lücken, Sabine Malzkuhn, Thomas Auth and Florian Wätjen. They have been there for me during the most difficult hours in my life, but also spent the most beautiful days with me. Their genuine care made me strong and ultimately helped me to write this thesis.

My husband Michael Hendrich deserves special thanks and words. Therefore, it was very tough to find the right words in english, so here are some words in eigener Sprache. Jeder Tag mit dir hat mich stärker gemacht. Ich freue mich nun sehr auf unsere gemeinsame Zukunft und bin gespannt darauf, was wir erreichen werden. 


\section{About the author}

Katharina Hendrich, born Dabow, *July 29, 1986, Sömmerda, German citizin.

\section{Curriculum Vitae}

2006 Abitur, Wilhelm-Hofmann-Gymnasium, Sankt Goarshausen.

2006-2009 Training as a chemistry laboratory assistant, DyStar Textilfarben $\mathrm{GmbH}$, Leverkusen.

2009 Chemistry laboratory assistant at Custom Manufactoring, DyStar Textilfarben GmbH, Leverkusen.

2009-2013 Bachelor of Science, Georg-August-University Göttingen, final grade: 2.0, Bachelor Thesis: "Schlenk-Gleichgewichte aluminiumorganischer Verbindungen".

2013-2016 Master of Science, Georg-August-University Göttingen, final grade: 1.9, Master Thesis: "Kinetische Untersuchungen zur Radikalischen Polymerisation von Captodativen Monomeren".

2016-2020 Doctoral Studies, Georg-August-University Göttingen, supervisor: Prof. Dr. Philipp Vana. 
